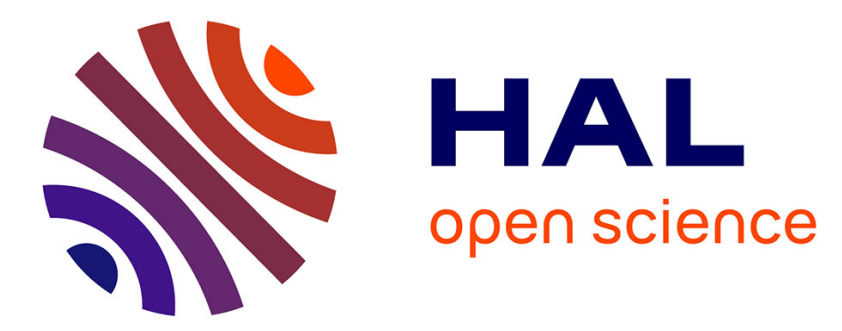

\title{
Loss of ellipticity analysis in non-smooth plasticity
}

\author{
B. Staber, Samuel Forest, M. Al Kotob, Matthieu Mazière, T. Rose
}

\section{To cite this version:}

B. Staber, Samuel Forest, M. Al Kotob, Matthieu Mazière, T. Rose. Loss of ellipticity analysis in non-smooth plasticity. International Journal of Solids and Structures, 2021, 222-223, pp.111010. 10.1016/j.ijsolstr.2021.02.021 . hal-03372375

\section{HAL Id: hal-03372375 \\ https://hal.science/hal-03372375}

Submitted on 16 Oct 2021

HAL is a multi-disciplinary open access archive for the deposit and dissemination of scientific research documents, whether they are published or not. The documents may come from teaching and research institutions in France or abroad, or from public or private research centers.
L'archive ouverte pluridisciplinaire HAL, est destinée au dépôt et à la diffusion de documents scientifiques de niveau recherche, publiés ou non, émanant des établissements d'enseignement et de recherche français ou étrangers, des laboratoires publics ou privés. 


\title{
Loss of ellipticity analysis in non-smooth plasticity
}

\author{
B. Staber ${ }^{\mathrm{a}, 1, *}$, S. Forest ${ }^{\mathrm{b}}$, M. Al Kotob ${ }^{\mathrm{c}}$, M. Mazire ${ }^{\mathrm{b}}$, T. Rose ${ }^{\mathrm{a}}$ \\ ${ }^{a}$ Safran Tech, Modelling and Simulation, Rue des Jeunes Bois, 78114 Magny-Les-Hameaux, France \\ ${ }^{b}$ MINES ParisTech, PSL University, Centre des matériaux, CNRS UMR 7633, BP 8791003 Evry, France \\ ${ }^{c}$ Safran Aircraft Engines Villaroche, Rond Point Ren Ravaud-Rau, 77550 Moissy-Cramayel, France
}

\begin{abstract}
This work is concerned with the influence of non-smooth yield functions on material instabilities. Using Rice's criterion, the conditions for loss of ellipticity are determined in the case of a family of non-smooth yield functions in principal stress space. It is found that the presence of corners on the yield surface allows for postponing or advancing loss of ellipticity. A procedure for the numerical detection of loss of ellipticity in multisurface plasticity is proposed. An explicit expression of the subdifferential of the non-smooth yield function is obtained, thereby extending existing results in the literature for the Tresca and MohrCoulomb criteria. Structural computations show that the use of non-smooth yield functions can lead to much earlier failure prediction than in the case of a commonly used von Mises criterion.
\end{abstract}

Keywords: loss of ellipticity, Rice's criterion, localization, non-smooth yield functions, corners, critical hardening modulus, Tresca

\section{Introduction}

This work is concerned with strain localization in the context of non-smooth plasticity. Introduced by [1] and [2], loss of ellipticity is commonly used as a criterion for the detection of the onset of strain localization. Loss of ellipticity is usually referred to as Rice's criterion ${ }_{5}$ for localization and corresponds to the existence of vanishing eigenvalues of the acoustic

\footnotetext{
${ }^{\star}$ Fully documented templates are available in the elsarticle package on CTAN,

*Corresponding author. Tel.: +33633999641

Email address: brian.staber@safrangroup.com (B. Staber) 
tensor. Loss of ellipticity is one criterion amongst others for the detection of instabilities. In particular, it has to be distinguished from loss of strong ellipticity which corresponds to the loss of positive definiteness of the symmetrized acoustic tensor. Classifications of instability criteria can be found in, e.g., [3, 4, 5, 6] amongst others. In a three-dimensional solid at small strains undergoing tension, loss of ellipticity is usually difficult to achieve unless the model exhibits a sufficiently strong softening behavior, i.e., a strongly negative hardening modulus. In addition, in the case of a smooth yield function, it has been shown by [7] that the critical hardening modulus for strain localization is never positive for associative plasticity at small strains. From a computational point of view, the detection of loss of ellipticity is recast as a minimization problem of the determinant of the acoustic tensor over the set of unit normals. Numerical strategies have been proposed in the literature by, e.g., [8]. More recently, a general and efficient multistart algorithm has been proposed for the detection of loss of ellipticity in elasto-plastic structures by [9].

Strain localization strongly depends on the plastic yield function. It is, for instance, well reported in the literature that strain localization predictions are highly sensitive to the formation of corners on the current yield surface. The phenomenon of vertex formation has been revealed by calculations based on crystal plasticity and by experimental evidence [10, 11]. Plasticity theories that take into account singularities on yield surfaces can roughly be divided into two categories, namely, phenomenological models based on the deformation theory of plasticity and computational-motivated models based on the classical flow theory of plasticity.

A first class of phenomenological corner theories has been proposed by [12. It is assumed that a corner exists on the yield surface and that it can be represented by a generalized cone in stress space. A general framework is proposed and then specialized to its simplest version known as the $\mathrm{J}_{2}$ corner theory. The model is built in such a way that it coincides with $\mathrm{J}_{2}$ deformation theory when stress increments are nearly proportional. This is achieved by taking the instantaneous moduli for nearly proportional loading equal to the tangent moduli of deformation theory. The aforementioned $\mathrm{J}_{2}$ corner theory has been used by [13, 14, 15, 16, 17, 18] for instability analysis at large strains and it is observed that a good 

corner theories were not well suited for large-scale simulations because they require one to track corners formation and their evolution. For these reasons, [19] has proposed a flow theory that mimics corner effects. A smooth yield surface and an associative flow rule are considered but the elasto-plastic moduli decrease as the deviation of the strain rate from proportional loading increases. Similarly, 20] has proposed a pseudo-corner theory using a smooth yield surface with a non-normality flow rule under the assumption that the plastic strain-rate and strain-rate deviator are coaxial. This model has been used in a few studies such as in 21] where classical flow, pseudo-corner and deformation theories are compared in a buckling analysis. Following these ideas, 22] extended the pseudo-corner model of [20] model [22] into strain-gradient plasticity. The common feature of all the aforementioned approaches is a reduction in the instantaneous shear moduli which in turn reduces the predicted bifurcation levels.

The extension of classical flow theory of plasticity to elastic domains formed by independent yield surfaces intersecting non-smoothly has first been carried out by [25]. The generalization to dependent yield surfaces has been achieved by [26] to accommodate crystal plasticity. This formulation of plasticity is commonly referred to as multisurface plasticity and its numerical treatment can be found in [27]. In metal plasticity, the standard threshold such as the Tresca criterion is covered by multisurface plasticity. A fully implicit integration algorithm for the Tresca criterion has been proposed by [28] by having recourse to an optimal parameterization in stress space. The extension of this approach to the Mohr-Coulomb criterion can be found in the textbook [29]. A generic fully implicit algorithm for multisurface models in principal stress space is presented by [30] together with a comprehensive review of methods for handling non-smooth yield surfaces. It should be noted that modern theory of plasticity is based on convex analysis as in, e.g., 31] and [32]. In this framework, the flow rule of plasticity is written as a differential inclusion involving the subdifferential set of the yield function and the initial boundary value problem is recast into an abstract variational inequality. As a result, non-smooth elastic domains can be considered and multisurface mod- 
els can be obtained as a special case. Theoretical aspects including mathematical theory and numerical analysis are presented by [33] in the context of small perturbations. Although the existence of solutions in finite deformations is still an open problem, recent advances can be found in [34] and [35] for the incremental boundary value problem. Very recently, a fully implicit subdifferential-based algorithm for the Mohr-Coulomb criterion has been devised by [36]. In contrast to multisurface-based algorithms, a single plastic Lagrange multiplier has to be computed and a simpler expression of the consistent tangent matrix can be determined.

The present paper makes the following contributions to the analysis of material instabilities: (a) The influence of non-smooth yield functions on the onset of loss of ellipticity is investigated by considering a family of yield functions that can be written as a linear combination of the principal stresses. Such a family includes classical yield functions such as the Tresca, twin shear stress [37], or the more recent mean influence factor model [38]. (b) It is shown that the presence and shape of corners on the yield surface allow for either postponing or advancing loss of ellipticity, or equivalently, decreasing or increasing the critical hardening modulus. (c) Based on the recent work of [9], a procedure for the numerical detection of loss of ellipticity in multisurface plasticity is proposed. (d) An explicit expression of the subdifferential set for the considered family of yield functions is provided, hence extending existing results in the literature for the Tresca and Mohr-Coulomb criteria [39, 40]. Such an expression is contained in the abstract form of the flow rule and can be used to, e.g., establish the multisurface form of the flow, or devise an implicit integration algorithm [40]. (e) Finite element simulations are performed on simple and realistic geometries in order to illustrate the proposed analysis. It is found that the choice of the yield function has a dramatic effect on the failure of structures. To the authors' best knowledge, such results are not yet reported in the literature.

This paper is organized as follows. First, in section 2, the theory of plasticity is presented in a convex analysis setting. In section 3 , the conditions for loss of ellipticity in multisurface plasticity are recalled. The family of yield functions considered in this work is described in section 4 which includes the definition of the flow rule in subdifferential and multisurface forms. The critical hardening moduli and shear bands orientations are then determined 
for stress states that lie on the smooth portions and corners of the yield surfaces. Finally, in section 5, numerical illustrations are provided in the case of a cube and a thin plate undergoing tension, and an experimental tubular specimen undergoing tension and torsion.

\section{Non-smooth plasticity}

In this section, we briefly recall the dual formulation of plasticity under the assumption of small strains. Comprehensive presentations of the theory of plasticity together with computational aspects at finite and small strains can be found in, e.g., [41] or [29]. Theoretical aspects including mathematical and numerical analysis are presented by [33] and the recent review 42 .

\subsection{Initial boundary value problem and flow rule}

Let $\Omega$ be a three-dimensional subset of $\mathbb{R}^{3}$ with Lipschitz boundary $\Gamma$ corresponding to the reference configuration occupied by the body of interest $\mathcal{B}$. The reference configuration undergoes a deformation map $\varphi: \Omega \times \mathcal{T} \rightarrow \mathbb{R}^{3}$ that results in a deformed configuration $\Omega_{t}$. Any point in the reference configuration is denoted by $\mathbf{x} \in \Omega$. We denote by $\mathbf{u}: \Omega \times \mathcal{T} \rightarrow \mathbb{R}^{3}$ the displacement field given by $\mathbf{u}(\mathbf{x}, t)=\boldsymbol{\varphi}(\mathbf{x}, t)-\mathbf{x}$ and let $\varepsilon: \Omega \times \mathcal{T} \rightarrow \mathbb{M}_{S}^{3}(\mathbb{R})$ be the second-order infinitesimal strain tensor given by

$$
\varepsilon(\mathbf{x}, t)=\frac{1}{2}\left(\mathbf{u}(\mathbf{x}, t) \otimes \nabla_{\mathbf{x}}+\nabla_{\mathbf{x}} \otimes \mathbf{u}(\mathbf{x}, t)\right) .
$$

Based on thermodynamic considerations, the constitutive equations are described in terms of the Helmholtz free energy function $\psi$. In order to account for plasticity, the strain tensor $\varepsilon$ is additively decomposed into an elastic contribution $\varepsilon^{e}$ and a plastic contribution $\varepsilon^{p}$, i.e.,

$$
\varepsilon(\mathbf{x}, t)=\varepsilon^{e}(\mathbf{x}, t)+\varepsilon^{p}(\mathbf{x}, t) .
$$

In order to account for hardening, we introduce a symmetric second-order tensor $\boldsymbol{\alpha}: \Omega \times \mathcal{T} \rightarrow$ $\mathbb{M}_{S}^{3}(\mathbb{R})$ which models kinematic hardening, and a vector $\mathbf{p}: \Omega \times \mathcal{T} \rightarrow \mathbb{R}^{m_{p}}$ which models isotropic hardening. Here, $m_{p} \geq 1$ is an integer that corresponds to the number of internal variables that model isotropic hardening. In most situations, isotropic hardening is described 
by a single internal variable, i.e., $m_{p}=1$. Multiple internal variables can be encountered in the cases of complex isotropic hardening behaviors or multisurface plasticity. For instance, in the case of single crystal plasticity, $m_{p}$ can be equal to the number of crystal systems. Let then $\mathbf{X}$ and $\mathbf{R}$ be the symmetric second-order tensor and the vector which are conjugate to $\boldsymbol{\alpha}$ and $\mathbf{p}$, respectively. The Helmholtz free energy function, assumed to be a function of the elastic strain tensor $\varepsilon^{e}:=\varepsilon-\varepsilon^{p}$ and of the hardening variables $\boldsymbol{\alpha}$ and $\mathbf{p}$, is further decomposed as

$$
\psi\left(\boldsymbol{\varepsilon}^{e}, \boldsymbol{\alpha}, \mathbf{p}\right)=\psi_{e}\left(\boldsymbol{\varepsilon}^{e}\right)+\psi_{p}(\boldsymbol{\alpha}, \mathbf{p})
$$

where $\psi_{e}$ and $\psi_{p}$ denote the elastic and inelastic contributions, respectively. In the context of linearized plasticity, the elastic free energy function is chosen as $\psi_{e}\left(\varepsilon^{e}\right)=(1 / 2) \varepsilon^{e}: \mathbb{C}: \varepsilon^{e}$ where $\mathbb{C}$ denotes the fourth-order elasticity tensor. Following the Clausius-Duhem inequality, the Cauchy stress tensor $\boldsymbol{\sigma}$ and the conjugate forces $\mathbf{X}$ and $\mathbf{R}$ are given by

$$
\boldsymbol{\sigma}=\mathbb{C}: \boldsymbol{\varepsilon}^{e}, \quad \mathbf{X}=-\frac{\partial \psi_{p}}{\partial \boldsymbol{\alpha}}, \quad \mathbf{R}=-\frac{\partial \psi_{p}}{\partial \mathbf{p}} .
$$

The second-order tensor $\mathbf{X}$ corresponds to the back-stress and the entries of the vector $\mathbf{R}=\left(R_{1}, \ldots, R_{m_{p}}\right)$ correspond to yield stresses that define the yield surface. In what follows, $\mathcal{S}$ and $\mathcal{P}$ denote the sets of generalized stresses and rate plastic variables such that $(\boldsymbol{\sigma}, \mathbf{X}, \mathbf{R}) \in \mathcal{S}$ and $\left(\dot{\boldsymbol{\varepsilon}}^{p}, \dot{\boldsymbol{\alpha}}, \dot{\mathbf{p}}\right) \in \mathcal{P}$. Arbitrary triplets in $\mathcal{P}$ and $\mathcal{S}$ will be denoted by $(\mathbf{A}, \mathbf{B}, \mathbf{c})$ and $\tilde{\boldsymbol{\sigma}}, \tilde{\mathbf{X}}, \tilde{\mathbf{R}}$, respectively. The evolution of the plastic strain tensor and hardening variables is described in terms of an elastic domain $\mathbb{E}$. Let then $N_{\mathbb{E}}(\boldsymbol{\sigma}, \mathbf{X}, \mathbf{R})$ be the normal cone to the elastic region $\mathbb{E}$ at $(\boldsymbol{\sigma}, \mathbf{X}, \mathbf{R}) \in \mathcal{S}$,

$$
N_{\mathbb{E}}(\boldsymbol{\sigma}, \mathbf{X}, \mathbf{R})=\{(\mathbf{A}, \mathbf{B}, \mathbf{c}) \in \mathcal{P} \mid \mathbf{A}:(\boldsymbol{\sigma}-\tilde{\boldsymbol{\sigma}})+\mathbf{B}:(\mathbf{X}-\tilde{\mathbf{X}})+\mathbf{c} \cdot(\mathbf{R}-\tilde{\mathbf{R}}) \geq 0, \forall(\tilde{\boldsymbol{\sigma}}, \tilde{\mathbf{X}}, \tilde{\mathbf{R}}) \in \mathcal{S}\}
$$

and let $\partial \phi$ be the multi-valued subdifferential set of the yield function, i.e.,

$$
\begin{aligned}
\partial \phi(\boldsymbol{\sigma}, \mathbf{X}, \mathbf{R})=\{(\mathbf{A}, \mathbf{B}, \mathbf{c}) \in \mathcal{P} \mid & \phi(\tilde{\boldsymbol{\sigma}}, \tilde{\mathbf{X}}, \tilde{\mathbf{R}}) \geq \phi(\boldsymbol{\sigma}, \mathbf{X}, \mathbf{R})+\mathbf{A}:(\tilde{\boldsymbol{\sigma}}-\boldsymbol{\sigma}) \\
& +\mathbf{B}:(\tilde{\mathbf{X}}-\mathbf{X})+\mathbf{c} \cdot(\tilde{\mathbf{R}}-\mathbf{R}), \forall(\tilde{\boldsymbol{\sigma}}, \tilde{\mathbf{X}}, \tilde{\mathbf{R}}) \in \mathcal{S}\} .
\end{aligned}
$$

The evolution of the plastic and internal variables are governed by the abstract flow rule

$$
\left(\dot{\boldsymbol{\varepsilon}}^{p}, \dot{\boldsymbol{\alpha}}, \dot{\mathbf{p}}\right) \in N_{\mathbb{E}}(\boldsymbol{\sigma}, \mathbf{X}, \mathbf{R}),
$$


which can be seen as an alternative formulation of the principle of maximum dissipation. The elastic domain is expressed in terms of a yield function $\phi$ as follows

$$
\mathbb{E}=\left\{(\tilde{\boldsymbol{\sigma}}, \tilde{\mathbf{X}}, \tilde{\mathbf{R}}) \in \mathcal{S} \mid \phi(\tilde{\boldsymbol{\sigma}}, \tilde{\mathbf{X}}, \tilde{\mathbf{R}}) \leq R_{0}\right\}
$$

where $R_{0}$ is a positive constant yield stress. It is assumed that $\mathbb{E}$ is a closed convex set and that it contains the origin, i.e., $\phi(\mathbf{0}, \mathbf{0}, \mathbf{0}) \leq R_{0}$. The abstract flow rule given by Eq. (7) can also be written in terms of the subdifferential set $\partial \phi$,

$$
\left(\dot{\boldsymbol{\varepsilon}}^{p}, \dot{\boldsymbol{\alpha}}, \dot{\mathbf{p}}\right) \in \dot{\gamma} \partial \phi(\boldsymbol{\sigma}, \mathbf{X}, \mathbf{R})
$$

where $\dot{\gamma} \geq 0$ is the plastic Lagrange multiplier. For given rates $\left(\dot{\boldsymbol{\varepsilon}}^{p}, \dot{\boldsymbol{\alpha}}, \dot{\mathbf{p}}\right)$, the Lagrange multipliers $\dot{\gamma}$ is given by the dissipation function $\mathcal{D}\left(\dot{\boldsymbol{\varepsilon}}^{p}, \dot{\boldsymbol{\alpha}}, \dot{\mathbf{p}}\right)$ defined as

$$
\mathcal{D}\left(\dot{\boldsymbol{\varepsilon}}^{p}, \dot{\boldsymbol{\alpha}}, \dot{\mathbf{p}}\right)=\sup \left\{\tilde{\boldsymbol{\sigma}}: \dot{\boldsymbol{\varepsilon}}^{p}+\tilde{\mathbf{X}}: \dot{\boldsymbol{\alpha}}+\tilde{\mathbf{R}} \cdot \dot{\mathbf{p}} \mid(\tilde{\boldsymbol{\sigma}}, \tilde{\mathbf{X}}, \tilde{\mathbf{R}}) \in \mathbb{E}\right\}
$$

which corresponds to the support function of the elastic region $\mathbb{E}$. Essential and natural boundary conditions are applied on the partitions $\Gamma_{D}$ and $\Gamma_{N}$ of $\Gamma$, with $\Gamma_{D} \cap \Gamma_{N}=\emptyset$ and $\Gamma=\Gamma_{D} \cup \Gamma_{N}$. Under the assumption of a quasi-static deformation process, the strong formulation of the initial boundary value problem takes the form

$$
\begin{aligned}
& -\nabla \cdot \boldsymbol{\sigma}=\mathbf{f}, \quad \text { in } \quad \Omega_{t} \times \mathcal{T}, \\
& \boldsymbol{\sigma} \mathbf{n}=\mathbf{t}_{d}, \quad \text { on } \quad \Gamma_{N} \times \mathcal{T}, \\
& \mathbf{u}=\mathbf{u}_{d}, \quad \text { in } \quad \Gamma_{D} \times \mathcal{T},
\end{aligned}
$$

where $\boldsymbol{\sigma}: \Omega \times \mathcal{T} \rightarrow \mathbb{M}_{S}^{3}(\mathbb{R})$ is the second-order Cauchy stress tensor, $\mathbf{f} \in \mathbb{R}^{3}$ describes the volume forces, and $\mathbf{n}$ is the outward unit normal at $\mathbf{x} \in \Gamma_{N}$.

\subsection{Multisurface plasticity deduced from convex analysis}

This section briefly describes how the multisurface flow rule can be deduced from the abstract flow rule given by Eq. (7). We consider elastic regions $\mathbb{E}$ formed by subsets $\mathbb{E}_{j} \subset \mathcal{S}$ that intersect non-smoothly, where $\mathcal{S}$ denotes the set of generalized stresses. Hence, the elastic domain is assumed to take the form $\mathbb{E}=\bigcap_{j=1}^{m} \mathbb{E}_{j}$ with

$$
\mathbb{E}_{j}=\left\{\left(\tilde{\boldsymbol{\sigma}}, \tilde{\mathbf{X}}, \tilde{R}_{j}\right) \in \mathcal{S} \mid \phi_{j}\left(\tilde{\boldsymbol{\sigma}}, \tilde{\mathbf{X}}, \tilde{R}_{j}\right)=\varphi_{j}(\tilde{\boldsymbol{\sigma}}-\tilde{\mathbf{X}})-\tilde{R}_{j} \leq 0\right\}, \quad j=1, \ldots, m,
$$


where $\phi_{j}$ and $\varphi_{j}$ are the $j$-th yield and canonical functions associated to $\mathbb{E}_{j}$. Under the assumption that there exists a family $\left\{\mu_{j}\right\}_{j=1}^{m}$ of positive real scalars such that

$$
\phi(\boldsymbol{\sigma}, \mathbf{X}, \mathbf{R})=\sum_{j=1}^{m} \mu_{j} \phi_{j}\left(\boldsymbol{\sigma}, \mathbf{X}, R_{j}\right)
$$

it can be shown that the subdifferential set $\partial \phi$ takes the form [43]

$$
\partial \phi(\boldsymbol{\sigma}, \mathbf{X}, \mathbf{R})=\left\{\mathbf{M} \in \mathbb{M}_{S}^{3} \mid \mathbf{M}=\sum_{j=1}^{m} \mu_{j} \mathbf{M}_{j}, \mathbf{M}_{j} \in \partial \phi_{j}\left(\boldsymbol{\sigma}, \mathbf{X}, R_{j}\right)\right\},
$$

where $\partial \phi_{j}$ is the subdifferential set associated to $\phi_{j}$. Assuming that the yield functions $\phi_{j}$, $j=1, \ldots, m$, are smooth functions of the generalized stresses, the subdifferential sets $\partial \phi_{j}$ are simply given by the singletons

$$
\partial \phi_{j}=\left\{\left(\dot{\boldsymbol{\varepsilon}}^{p}, \dot{\boldsymbol{\alpha}}, \dot{p}_{j}\right)=\left(\nabla_{\boldsymbol{\sigma}} \phi_{j}, \nabla_{\mathbf{X}} \phi_{j}, \nabla_{R_{j}} \phi_{j}\right)\right\}, \quad j=1, \ldots, m
$$

and the abstract flow rule given by Eq. (9) reduces to

$$
\dot{\boldsymbol{\varepsilon}}^{p}=\sum_{j=1}^{m} \dot{\gamma}_{j} \nabla_{\boldsymbol{\sigma}} \phi_{j}, \quad \dot{\boldsymbol{\alpha}}=-\sum_{j=1}^{m} \dot{\gamma}_{j} \nabla_{\mathbf{X}} \phi_{j}, \quad \dot{\mathbf{p}}=-\sum_{j=1}^{m} \dot{\gamma}_{j} \nabla_{\mathbf{R}} \phi_{j},
$$

which corresponds to Koiter's form of the flow rule, with $\dot{\gamma}_{j}=\dot{\gamma} \mu_{j}$. The Lagrange multipliers $\dot{\gamma}_{1}, \ldots, \dot{\gamma}_{m}$ satisfy the Kuhn-Tucker complementary conditions

$$
\dot{\gamma}_{j} \geq 0, \quad \phi_{j}\left(\boldsymbol{\sigma}, \mathbf{X}, R_{j}\right) \leq 0, \quad \dot{\gamma}_{j} \phi_{j}\left(\boldsymbol{\sigma}, \mathbf{X}, R_{j}\right)=0
$$

for $j=1, \ldots, m$. Eq. 16 complemented by the conditions given by Eq. (17) is referred to as a multisurface model for continuously differentiable yield functions that intersect nonsmoothly. Using the constitutive equations given by Eq. (4) together with the chosen form of the canonical yield function, one has the following constitutive rate equations

$$
\dot{\boldsymbol{\sigma}}=\mathbb{C}: \dot{\boldsymbol{\varepsilon}}-\sum_{j=1}^{m} \mathbb{C}: \mathbf{N}_{j} \dot{\gamma}_{j}, \quad \dot{\mathbf{X}}=\sum_{j=1}^{m} \mathbb{K}(\boldsymbol{\alpha}): \mathbf{N}_{j} \dot{\gamma}_{j}, \quad \dot{R}_{i}=\sum_{j=1}^{m} H_{i j}(\mathbf{p}) \dot{\gamma}_{j}
$$

where $\mathbf{N}_{i}=\nabla_{\boldsymbol{\sigma}} \phi_{i}$ is the normal tensor to the $i$-th yield surface, $\mathbb{K}=\nabla_{\boldsymbol{\alpha}}^{2} \psi_{p}$ and $[H]=\nabla_{\mathbf{p}}^{2} \psi_{p}$ are the kinematic and isotropic hardening moduli, respectively. The multisurface form of 
the flow rule allows for eliminating the Lagrange multipliers $\dot{\gamma}_{1}, \ldots, \dot{\gamma}_{m}$ by invoking the consistency conditions $\dot{\gamma}_{j} \dot{\phi}_{j}(\boldsymbol{\sigma}, \mathbf{X}, \mathbf{R})=0, j=1, \ldots, m$. By successively applying the chain rule, it is found that

$$
\dot{\phi}_{j}(\boldsymbol{\sigma}, \mathbf{X}, \mathbf{R})=\nabla_{\boldsymbol{\sigma}} \phi_{j}(\boldsymbol{\sigma}, \mathbf{X}, \mathbf{R}): \dot{\boldsymbol{\sigma}}+\nabla_{\mathbf{X}} \phi_{j}(\boldsymbol{\sigma}, \mathbf{X}, \mathbf{R}): \dot{\mathbf{X}}+\nabla_{R_{j}} \phi_{j}\left(\boldsymbol{\sigma}, \mathbf{X}, R_{j}\right) \dot{R}_{j}
$$

which together with Eq. (18), leads to the following possibly non-linear system of equations for $\left(\dot{\gamma}_{1}, \ldots, \dot{\gamma}_{m}\right)$,

$$
[G(\boldsymbol{\alpha}, \mathbf{p})] \dot{\lambda}=\mathbf{b}(\dot{\varepsilon})
$$

where $\dot{\boldsymbol{\lambda}}$ is the vector gathering the Lagrange multipliers, that is, $\dot{\boldsymbol{\lambda}}=\left(\dot{\gamma}_{1}, \ldots, \dot{\gamma}_{m}\right)$. The entries of the matrix $[A]$ and vector $\mathbf{b}$ are given by

$$
G_{i j}(\boldsymbol{\alpha}, \mathbf{p})=\chi_{i j}+\varsigma_{i j}(\boldsymbol{\alpha})+H_{i j}(\mathbf{p}), \quad b_{i}(\dot{\boldsymbol{\varepsilon}})=\mathbf{N}_{i}: \mathbb{C}: \dot{\boldsymbol{\varepsilon}}, \quad 1 \leq i, j \leq m,
$$

where $\chi_{i j} \equiv \mathbf{N}_{i}: \mathbb{C}: \mathbf{N}_{j}$ and $\varsigma_{i j}(\boldsymbol{\alpha})=\mathbf{N}_{i}: \mathbb{K}(\boldsymbol{\alpha}): \mathbf{N}_{j}$. While the Lagrange multiplier $\dot{\gamma}$ in Eq. (9) can be written as $\dot{\gamma}=\mathcal{D}\left(\dot{\varepsilon}^{p}, \dot{\boldsymbol{\alpha}}, \dot{\mathbf{p}}\right)$, it can also be obtained through the relation $\dot{\gamma}=\sum_{j=1}^{m} \dot{\gamma}_{j}$ under the assumption that Eq. 13) holds with the additional constraint that $\sum_{j=1}^{m} \mu_{j}=1$. Under the assumption that there exists at least one solution to the problem defined by Eq. (20), the elasto-plastic tangent tensor $\mathbb{C}^{e p}$, defined such that

$$
\dot{\boldsymbol{\sigma}}=\mathbb{C}^{e p}: \dot{\boldsymbol{\varepsilon}}
$$

is given by

$$
\mathbb{C}^{e p}=\mathbb{C}-\sum_{j=1}^{m} \mathbb{C}: \mathbf{N}_{j} \otimes \tilde{\mathbf{N}}_{j}: \mathbb{C}, \quad \tilde{\mathbf{N}}_{j}=\sum_{k=1}^{m} G_{j k}^{-1}(\boldsymbol{\alpha}, \mathbf{p}) \mathbf{N}_{k},
$$

where $G_{i j}^{-1}(\boldsymbol{\alpha}, \mathbf{p})$ for $1 \leq i, j \leq m$ denote the entries of the inverse matrix $[G(\boldsymbol{\alpha}, \mathbf{p})]^{-1}$. The elasto-plastic tangent tensor plays a fundamental role in the prediction of strain localization.

\section{Conditions for loss of ellipticity in multisurface plasticity}

This section is concerned with material instabilities due to the development of weak discontinuities. It is assumed that there exists a singular surface $\Gamma_{s}$ of order one which is 
characterized by a jump of the deformation rate and a continuous rate of the traction vector. We let $\mathbf{n} \in \mathscr{S}$ be the normal to the singular surface $\Gamma_{s}$, and define the sets $\Gamma_{s}^{-}$and $\Gamma_{s}^{+}$as

$$
\Gamma_{s}^{-}=\left\{\mathbf{x} \in \Omega \mid(\mathbf{x}-\mathbf{y}) \mathbf{n}<0, \forall \mathbf{y} \in \Gamma_{s}\right\}, \quad \Gamma_{s}^{+}=\left\{\mathbf{x} \in \Omega \mid(\mathbf{x}-\mathbf{y}) \mathbf{n}>0, \forall \mathbf{y} \in \Gamma_{s}\right\}
$$

For any function $f$ and a point $\mathbf{p} \in \Gamma_{s}$, we let $f^{-}$and $f^{+}$be the left and right limits given by

$$
f^{-}(\mathbf{y})=\lim _{\mathbf{x} \rightarrow \mathbf{y}, \mathbf{x} \in \Gamma_{s}^{-}} f(\mathbf{x}), \quad f^{+}(\mathbf{y})=\lim _{\mathbf{x} \rightarrow \mathbf{y}, \mathbf{x} \in \Gamma_{s}^{+}} f(\mathbf{x}),
$$

and the jump of the function $f$ at $\mathbf{y} \in \Gamma_{s}$ is given by $\llbracket f(\mathbf{y}) \rrbracket \equiv f^{+}(\mathbf{y})-f^{-}(\mathbf{y})$. The conditions for a first-order singular surface can then be written as

$$
\llbracket \nabla \dot{\mathbf{u}} \rrbracket=\rho \mathbf{g} \otimes \mathbf{n}, \quad \llbracket \dot{\boldsymbol{\sigma}} \mathbf{n} \rrbracket=\mathbf{0} .
$$

We restrict ourselves to the case of continuous localization which is characterized by a continuous tangent elasto-plastic operator across the singular surface. Using the rate constitutive equation $\dot{\boldsymbol{\sigma}}=\mathbb{C}^{e p}: \dot{\boldsymbol{\varepsilon}}$ together with Eq. (26), it can be deduced that there exists a non-zero $\mathbf{m} \in \mathscr{S}$ such that

$$
\mathrm{Q}_{e p}(\mathbf{n}) \cdot \mathbf{g}=\mathbf{0}
$$

where $\mathbf{Q}_{e p}(\mathbf{n})$ is the elasto-plastic acoustic tensor defined as $\mathbf{Q}_{e p}(\mathbf{n}) \equiv \mathbb{C}^{e p} \circ(\mathbf{n} \otimes \mathbf{n})$ with $(\mathbb{A} \circ \mathbf{B})_{i k}=\mathbb{A}_{i j k \ell} B_{j \ell}$. We recall that two situations can be encountered (see [7]): split instability whenever $\mathbf{g} \propto \mathbf{n}$ and shear band instability whenever $\mathbf{g} \not<\mathbf{n}$.

\subsection{Case of a single active mechanism}

We first assume that the current stress state lies on a smooth portion of the yield surface, i.e., $\varphi_{K}=0$ for some $K \in\{1, \ldots, m\}$. In this case, the tangent elasto-plastic tensor given by Eq. (23) reduces to

$$
\mathbb{C}^{e p}=\mathbb{C}-\frac{\left(\mathbb{C}: \mathbf{N}_{K}\right) \otimes\left(\mathbf{N}_{K}: \mathbb{C}\right)}{\chi_{K K}+H_{K K}}, \quad \chi_{K K}=\mathbf{N}_{K}: \mathbb{C}: \mathbf{N}_{K}
$$

By injecting the above expression into the acoustic tensor, using the $\operatorname{condition} \operatorname{det}\left(\mathbf{Q}_{e p}(\mathbf{n})\right)=$ 0 , and assuming that the hardening modulus decreases with the plastic strain, the following 
critical hardening modulus is obtained

$$
H_{K}^{c r i t}(\mathbf{n})=\max _{\mathbf{n},\|\mathbf{n}\|=1}\left\langle\mathbf{d}(\mathbf{n}), \mathbf{Q}_{e l}^{-1}(\mathbf{n}) \mathbf{d}(\mathbf{n})\right\rangle-\mathbf{N}_{K}: \mathbb{C}: \mathbf{N}_{K}
$$

with $\mathbf{d}(\mathbf{n})=\left(\mathbb{C}: \mathbf{N}_{K}\right) \mathbf{n}$. For an isotropic material, the elastic acoustic tensor is given by $\mathbf{Q}_{e l}(\mathbf{n})=(\lambda+2 \mu) \mathbf{n} \otimes \mathbf{n}+\mu([1]-\mathbf{n} \otimes \mathbf{n})$ and its inverse takes the form

$$
\mathbf{Q}_{e l}^{-1}(\mathbf{n})=-\frac{(\lambda+\mu)}{\mu(\lambda+2 \mu)} \mathbf{n} \otimes \mathbf{n}+\frac{1}{\mu}[1] .
$$

Let then $\left\{N_{j}^{K}\right\}_{j=1}^{3}$ be the set of eigenvalues of $\mathbf{N}^{K}$. The optimization problem given by Eq. 29] is equivalent to the maximization of a Lagrangian function, leading to

$$
n_{j}\left(\left(N_{j}^{K}\right)^{2}-\frac{1}{1-\nu} \sum_{i=1}^{3}\left(n_{i}^{2} N_{i}^{K}\right) N_{j}^{K}-\frac{\beta}{4 \mu}\right)=0, \quad j \in\{1,2,3\},
$$

where $\beta$ is a Lagrange multiplier associated to the normalisation constraint $\|\mathbf{n}\|^{2}=1$. Different cases have to be distinguished depending on the values of $n_{1}, n_{2}$, and $n_{3}$ and the following solutions can be found (see [7]). If $n_{1}, n_{2}$, and $n_{3}$ are non-zero, then the system is indeterminate and there are no solutions. If $n_{i} \neq 0, n_{j} \neq 0, n_{k}=0$, and $n_{i} \neq n_{j}$, then

$$
H_{K}^{c r i t}(\mathbf{n})=-E\left(N_{k}^{K}\right)^{2}, \quad n_{i}^{2}=\frac{N_{i}^{K}+\nu N_{k}^{K}}{N_{i}^{K}-N_{j}^{K}}, \quad n_{j}^{2}=1-n_{i}^{2} .
$$

In this case, the eigenvector $\mathbf{g}$ in Eq. 27) can be decomposed onto the principal basis as $\mathbf{g}=\sum_{i=1}^{3} g_{i} \mathbf{m}_{i} \otimes \mathbf{m}_{i}$ where the components $g_{1}, g_{2}, g_{3}$ are given by

$$
g_{i}=\left(N_{i}^{K}-N_{j}^{K}\right) n_{i}, \quad g_{j}=\left(N_{j}^{K}-N_{i}^{K}\right) n_{j}, \quad g_{k}=0 .
$$

Finally, if $n_{i}=1$ and $n_{j}=n_{k}=0$, then

$$
H_{K}^{c r i t}(\mathbf{n})=-2 \mu\left(\frac{\left(N_{j}^{K}+\nu N_{k}^{K}\right)^{2}}{1-\nu}+(1+\nu)\left(N_{k}^{K}\right)^{2}\right) .
$$

As a result, the critical hardening modulus for strain localization is never strictly positive whenever a single mechanism is active. 


\subsection{Case of multiple active mechanisms}

We now consider the case where the current stress state lies on a corner of the yield surface, resulting in multiple active mechanisms. Using the general expression of $\mathbb{C}^{e p}$ given by Eq. (23) for multisurface plasticity, the acoustic tensor takes the form

$$
\mathbf{Q}_{e p}(\mathbf{n})=\mathbf{Q}_{e l}(\mathbf{n})-\sum_{J \in \mathbb{J}}\left(\left(\mathbb{C}: \mathbf{N}_{I}\right) \mathbf{n}\right) \otimes\left(\left(\tilde{\mathbf{N}}_{I}: \mathbb{C}\right) \mathbf{n}\right)
$$

where $\mathbf{Q}_{e l}(\mathbf{n}) \equiv \mathbb{C} \circ(\mathbf{n} \otimes \mathbf{n})$ denotes the elastic acoustic tensor, $\mathbb{J}$ is the set of $m_{\text {act }}$ active mechanisms, and $\tilde{\mathbf{N}}_{I}$ is defined in Eq. 23). Under the assumption that the hardening matrix $[H]$ is symmetric, the acoustic tensor is parameterized by $m\left(m_{\text {act }}+1\right) / 2$ hardening moduli $H_{i j}$. Let $\overline{\mathbf{Q}}_{e p}(\mathbf{n})$ be the normalized acoustic tensor defined such that $\mathbf{Q}_{e p}(\mathbf{n})=\mathbf{Q}_{e l}(\mathbf{n}) \overline{\mathbf{Q}}_{e p}(\mathbf{n})$, that is

$$
\overline{\mathbf{Q}}_{e p}(\mathbf{n})=[1]-\sum_{J \in \mathbb{J}} \mathbf{Q}_{e l}^{-1}(\mathbf{n})\left(\left(\mathbb{C}: \mathbf{N}_{I}\right) \mathbf{n}\right) \otimes\left(\left(\tilde{\mathbf{N}}_{I}: \mathbb{C}\right) \mathbf{n}\right) .
$$

It follows that the determinant of the acoustic tensor can be written as $\operatorname{det}\left(\mathbf{Q}_{e p}(\mathbf{n})\right)=$ $\operatorname{det}\left(\mathbf{Q}_{e l}(\mathbf{n})\right) \operatorname{det}\left(\overline{\mathbf{Q}}_{e p}(\mathbf{n})\right)$. Under the assumption that $\operatorname{det}\left(\mathbf{Q}_{e l}(\mathbf{n})\right) \neq 0$, the condition for the onset of continuous localization given by Eq. (27) is rewritten as: Find $\mathbf{n} \in \mathscr{S}$ such that

$$
\operatorname{det}\left(\overline{\mathbf{Q}}_{e p}(\mathbf{n})\right)=0
$$

Let $[\pi(\mathbf{n})]$ be the $m_{\text {act }} \times m_{\text {act }}$ matrix defined component-wise as

$$
\pi_{I J}(\mathbf{n}) \equiv\left\langle\left(\mathbf{N}_{K}: \mathbb{C}\right) \mathbf{n}, \mathbf{Q}_{e l}^{-1}(\mathbf{n})\left(\mathbf{N}_{L}: \mathbb{C}\right) \mathbf{n}\right\rangle, \quad 1 \leq I, J \leq m_{\text {act }}, \quad(K, L) \in \mathbb{J} \times \mathbb{J},
$$

where it is recalled that $m_{\text {act }}=\operatorname{Card}(\mathbb{J})$ denotes the number of active yield surfaces. Using the expression of $\mathbf{Q}_{e l}^{-1}(\mathbf{n})$, the matrix $[\boldsymbol{\pi}(\mathbf{n})$ ] defined by Eq. (38) can be rewritten as

$$
\pi_{I J}(\mathbf{n})=-\frac{4 \mu(\lambda+\mu)}{\lambda+2 \mu}\left\langle\mathbf{n}, \mathbf{N}_{K} \mathbf{n}\right\rangle\left\langle\mathbf{n}, \mathbf{N}_{L} \mathbf{n}\right\rangle+4 \mu\left\langle\mathbf{N}_{K} \mathbf{n}, \mathbf{N}_{L} \mathbf{n}\right\rangle
$$

By analyzing the eigenvalue problem for the update matrix in Eq. (36), it can be deduced that

$$
\operatorname{det}\left(\overline{\mathbf{Q}}_{e p}(\mathbf{n})\right)=\operatorname{det}\left([1]-[G]^{-1}[\pi(\mathbf{n})]\right), \quad[G]=[\chi]+[H] .
$$


Under the assumption that $[G]$ is positive definite (see Eqs. (20)-(21)), it follows that the condition for the onset of localization given by Eq. (37) is equivalent to finding $\mathbf{n} \in \mathscr{S}$ such that

$$
\operatorname{det}([\pi(\mathbf{n})]-([\chi]+[H]))=0 .
$$

This condition has been obtained by [44] and 45] for small and large strains, respectively, and explicit expressions of the critical hardening moduli were obtained for a specific family of hardening matrix. Herein, we introduce the eigenvalues $N_{i}^{K}, N_{i}^{L}$ and $n_{i}$ of $\mathbf{N}^{K}, \mathbf{N}^{L}$, and $\mathbf{n}$ in the principal stress basis $\left(\mathbf{m}_{1}, \mathbf{m}_{2}, \mathbf{m}_{3}\right)$. The entries of the matrix $[\pi(\mathbf{n})]$ can then be written as $\hat{\pi}_{I J}\left(n_{1}, n_{2}, n_{3}\right)=\pi_{I J}(\mathbf{n})$ with

$$
\hat{\pi}_{I J}\left(n_{1}, n_{2}, n_{3}\right)=-\frac{4 \mu(\lambda+\mu)}{\lambda+2 \mu} \sum_{i=1}^{3} n_{i}^{2} N_{i}^{K} \sum_{j=1}^{3} n_{j}^{2} N_{j}^{L}+4 \mu \sum_{i=1}^{3} N_{i}^{K} N_{i}^{L} n_{i}^{2}, \quad \mathbf{n}=\sum_{k=1}^{3} n_{k} \mathbf{m}_{k} .
$$

Unfortunately, solving Eq. 41] for the entries of $[H]$ and for any $m_{\text {act }} \geq 2$ seems to be analytically intractable. In the sequel of this paper, at most two simultaneous active yield surfaces will be considered. Hence, we consider $m_{a c t}=2$ and if $[\pi(\mathbf{n})]-[\chi]$ is invertible, Eq. (41) reduces to

$$
\operatorname{det}([\pi(\mathbf{n})]-[\chi]-[H])=\operatorname{det}([\pi(\mathbf{n})]-[\chi])+\operatorname{det}([H])+\operatorname{adj}([\pi(\mathbf{n})]-[\chi]):[H] .
$$

In this case, explicit expressions of the critical hardening moduli are given by [44 for the family of matrices $[H(\ell)]$, that is,

$$
[H(\ell)] \equiv H_{\ell}\left(\ell\left[i_{m}\right]+(1-\ell)[1]\right)
$$

where $H_{\ell}$ is a scalar hardening modulus and $\left[i_{m}\right]_{I J}=1$ for all $I, J \in\left\{1, \ldots, m_{\text {act }}\right\}^{2}$. Three cases can be distinguished.

(a) For $\ell=1$, it is found that $\operatorname{det}([H(\ell)])=0$ and Eq. (43) reduces to

$$
\operatorname{det}([\pi(\mathbf{n})]-[\chi])-H_{1} \operatorname{adj}([\pi(\mathbf{n})]-[\chi]):\left[i_{m}\right]=0 .
$$

Hence, it can be deduced that the critical scalar hardening modulus $H_{1}^{\text {crit }}$ is given by

$$
H_{1}^{c r i t}=\max _{\|\mathbf{n}\|=1}\left(([\pi(\mathbf{n})]-[\chi])^{-1}:\left[i_{m}\right]\right)^{-1} .
$$


(b) For $\ell=0$, it is found that

$$
\left.H_{0}^{2}-H_{0} \operatorname{adj}([\pi(\mathbf{n})]-[\chi])\right):[1]+\operatorname{det}([\pi(\mathbf{n})]-[\chi])=0,
$$

which corresponds to the characteristic equation for $[\pi(\cdot)]-[\chi]$ with $H_{0}$ as an eigenvalue. Hence, the critical hardening modulus is given by

$$
H_{0}^{\text {crit }}=\max _{\|\mathbf{n}\|=1} \max _{K} \lambda_{K}([\pi(\mathbf{n})]-[\chi])
$$

(c) For an arbitrary $\ell$ such that $\ell \neq 0$ and $\ell \neq 1$, one has

$$
a_{\ell} H_{\ell}^{2}+b_{\ell}(\mathbf{n}) H_{\ell}+c(\mathbf{n})=0
$$

where $a_{\ell}=\left(1-\ell^{2}\right), c(\mathbf{n})=\operatorname{det}([\pi(\mathbf{n})]-[\chi])$, and

$$
b_{\ell}(\mathbf{n})=\operatorname{ladj}([\pi(\mathbf{n})]-[\chi]):\left[i_{m}\right]+(1-\ell) \operatorname{adj}([\pi(\mathbf{n})]-[\chi]):[1]
$$

Given a normal $\mathbf{n}$, the above second-order equation can easily be solved for the hardening modulus, yielding $\mathbf{n} \mapsto H_{\ell}(\mathbf{n})$, in order to deduce the critical hardening modulus $H_{\ell}^{\text {crit }}=$ $\max _{\|\mathbf{n}\|=1} H_{\ell}(\mathbf{n})$. Different cases have to be distinguished depending on the value of the parameter $\ell$.

Remark 1. It should be emphasized that the analysis presented in this section is valid under the constraint that the matrix $[G]$ defined by Eq. (21) is nonsingular. For $\ell \geq 0$ and $\ell \neq 1$, the matrix $[G]=[\chi]+[H(\ell)]$ loses positive definiteness if

$$
H_{\ell}=\frac{2 \mu}{\ell-1}, \quad \text { or } \quad H_{\ell}=-\frac{6 \mu}{\ell+1},
$$

and for $\ell=1$,

$$
H_{\ell}=-3 \mu
$$

All these critical values are negative for $\ell \leq 1$. However, in the case of latent hardening for which $\ell>1$, the first critical modulus in Eq. (51) is positive. 


\subsection{Numerical detection of loss of ellipticity in non-smooth plasticity}

In this section, the recent algorithm proposed by [9] for the detection of loss of ellipticity is specified for multisurface plasticity. The algorithm draws on the approach proposed by [8] together with an efficient multistart strategy. The optimization problem raised by the condition of the onset of loss of ellipticity is recast into the minimization problem: Find $\mathbf{n}^{\star}$ such that

$$
\operatorname{det}\left(\left[\pi\left(\mathbf{n}^{\star}\right)\right]-[\chi]-[H]\right)=\min _{\mathbf{n} \in \mathcal{S}} \operatorname{det}([\pi(\mathbf{n})]-[\chi]-[H]) .
$$

Let then $\mathbf{r}: \mathcal{S} \rightarrow \mathbb{R}^{3}$ be the residual vector defined as

$$
\mathbf{r}(\mathbf{n})=\frac{\partial}{\partial \mathbf{n}} \operatorname{det}([\pi(\mathbf{n})]-[\chi]-[H]) .
$$

For notational convenience, we let $[\tilde{\pi}(\cdot)]=[\pi(\cdot)]-[\chi]-[H]$. The following identities will be useful for the developments of this section:

$$
\frac{\partial \operatorname{det}([\tilde{\pi}])}{\partial[\tilde{\pi}]}=\operatorname{det}([\tilde{\pi}])\left([\tilde{\pi}]^{-1}\right)^{T}, \quad \frac{\partial[\tilde{\pi}]^{-1}}{\partial[\tilde{\pi}]}=-[\tilde{\pi}]^{-1} \otimes[\tilde{\pi}]^{-1}
$$

and the adjugate matrix $\operatorname{det}([\tilde{\pi}])[\tilde{\pi}]^{-1}$ will be denoted as $\operatorname{adj}([\tilde{\pi}])$. It can then be $\operatorname{deduced}$ that the residual $\mathbf{r}$ vector takes the form

$$
\mathbf{r}(\mathbf{n})=[\operatorname{ajd}([\tilde{\pi}(\mathbf{n})])]: \frac{\partial[\pi(\mathbf{n})]}{\partial \mathbf{n}},
$$

where the first-order partial derivatives of $[\pi(\mathbf{n})]$ with respect to $\mathbf{n}$ are given by

$\frac{\partial \pi_{I J}(\mathbf{n})}{\partial \mathbf{n}}=\alpha_{1}\left\langle\mathbf{n}, \mathbf{N}_{I} \mathbf{n}\right\rangle\left(\mathbf{N}_{J} \mathbf{n}+\mathbf{N}_{J}^{T} \mathbf{n}\right)+\alpha_{1}\left\langle\mathbf{n}, \mathbf{N}_{J} \mathbf{n}\right\rangle\left(\mathbf{N}_{I} \mathbf{n}+\mathbf{N}_{I}^{T} \mathbf{n}\right)+\alpha_{2}\left(\mathbf{N}_{I}^{T} \mathbf{N}_{J} \mathbf{n}+\mathbf{N}_{J}^{T} \mathbf{N}_{I} \mathbf{n}\right)$,

for $I, J \in \mathbb{J} \times \mathbb{J}$ and with $\alpha_{1}=-4 \mu(\lambda+\mu) /(\lambda+2 \mu)$ and $\alpha_{2}=4 \mu$. The first-order derivative of $\mathbf{r}$ with respect to $\mathbf{n}$ is given by

$$
\begin{aligned}
{[K(\mathbf{n})]=} & \frac{\mathbf{r}(\mathbf{n}) \otimes \mathbf{r}(\mathbf{n})}{\operatorname{det}([\tilde{\pi}(\mathbf{n})])}-\operatorname{det}([\tilde{\pi}(\mathbf{n})]) \frac{\partial[\pi(\mathbf{n})]}{\partial \mathbf{n}}:\left([\tilde{\pi}(\mathbf{n})]^{-1} \otimes[\tilde{\pi}(\mathbf{n})]^{-1}\right): \frac{\partial[\pi(\mathbf{n})]}{\partial \mathbf{n}} \\
& +[\operatorname{ajd}([\tilde{\pi}(\mathbf{n})])]: \frac{\partial^{2}[\pi(\mathbf{n})]}{\partial \mathbf{n} \otimes \partial \mathbf{n}},
\end{aligned}
$$

where the second-order partial derivatives of $[\pi(\mathbf{n})]$ are given by

$$
\begin{aligned}
\frac{\partial^{2} \pi_{I J}(\mathbf{n})}{\partial \mathbf{n} \otimes \partial \mathbf{n}} & =\alpha_{1}\left(\mathbf{N}_{I} \mathbf{n}+\mathbf{N}_{I}^{T} \mathbf{n}\right) \otimes\left(\mathbf{N}_{J} \mathbf{n}+\mathbf{N}_{J}^{T} \mathbf{n}\right)+\alpha_{1}\left(\mathbf{N}_{J} \mathbf{n}+\mathbf{N}_{J}^{T} \mathbf{n}\right) \otimes\left(\mathbf{N}_{I} \mathbf{n}+\mathbf{N}_{I}^{T} \mathbf{n}\right) \\
& +\alpha_{1}\left\langle\mathbf{n}, \mathbf{N}_{I} \mathbf{n}\right\rangle\left(\mathbf{N}_{J}+\mathbf{N}_{J}^{T}\right)+\alpha_{1}\left\langle\mathbf{n}, \mathbf{N}_{J} \mathbf{n}\right\rangle\left(\mathbf{N}_{I}+\mathbf{N}_{I}^{T}\right)+\alpha_{2}\left(\mathbf{N}_{I}^{T} \mathbf{N}_{J}+\mathbf{N}_{J}^{T} \mathbf{N}_{I}\right)
\end{aligned}
$$


Since the normal vector $\mathbf{n}$ is normalized, it can be expressed in terms of polar $\theta_{1}$ and azimuthal $\theta_{2}$ angles as $\mathbf{n}=\hat{\mathbf{n}}(\boldsymbol{\theta})$ with $\hat{\mathbf{n}}(\boldsymbol{\theta})=\left(\cos \left(\theta_{1}\right) \sin \left(\theta_{2}\right), \sin \left(\theta_{1}\right) \sin \left(\theta_{2}\right), \cos \left(\theta_{2}\right)\right)$ in the canonical basis of $\mathbb{R}^{3}$. Hence, the minimization problem can be rewritten as

$$
\operatorname{det}\left(\left[\hat{\pi}\left(\boldsymbol{\theta}^{\star}\right)\right]-[\chi]-[H]\right)=\min _{\boldsymbol{\theta} \in \Theta} \operatorname{det}([\hat{\pi}(\boldsymbol{\theta})]-[\chi]-[H]),
$$

with $[\hat{\pi}(\boldsymbol{\theta})]=[\pi(\hat{\mathbf{n}}(\boldsymbol{\theta}))]$. Using the chain rule, the associated residual vector $\hat{\mathbf{R}}: \Theta \rightarrow \mathbb{R}^{3}$ is given by

$$
\hat{R}_{i}(\boldsymbol{\theta})=D_{i j}(\boldsymbol{\theta})^{T} R_{j}(\hat{\mathbf{n}}(\boldsymbol{\theta})), \quad D_{i j}(\boldsymbol{\theta})=\frac{\partial \hat{n}_{i}(\boldsymbol{\theta})}{\partial \theta_{j}} .
$$

The problem of finding $\boldsymbol{\theta} \in \Theta$ such that $\hat{\mathbf{R}}(\boldsymbol{\theta})=\mathbf{0}$ is solved with a Newton-Raphson scheme. Given an initial guess $\boldsymbol{\theta}^{(0)} \in \Theta$, let $\boldsymbol{\theta}^{(k)}$ for $k \geq 1$ be a sequence such that

$$
k \geq 0: \quad\left[\hat{K}\left(\boldsymbol{\theta}^{(k)}\right)\right]\left(\boldsymbol{\theta}^{(k+1)}-\boldsymbol{\theta}^{(k)}\right)=-\mathbf{R}\left(\boldsymbol{\theta}^{k}\right),
$$

where the tangent matrix $[\hat{K}]$ is given component-wise by

$$
\hat{K}_{i j}(\boldsymbol{\theta})=D_{i k}^{T}(\boldsymbol{\theta}) K_{k l}(\hat{\mathbf{n}}(\boldsymbol{\theta})) D_{l k}(\boldsymbol{\theta})+\hat{R}_{k}(\boldsymbol{\theta}) \frac{\partial D_{k i}(\boldsymbol{\theta})}{\partial \theta_{j}} .
$$

Loss of ellipticity is detected at each gauss point of the finite element using the NewtonRaphson procedure given by Eq. 62). In practice, a set of initial guesses $\left\{\boldsymbol{\theta}^{(0, s)}\right\}_{s=1}^{M}$ is first computed by discretization of the unit sphere. The minimization problem is solved for each element of this set and the solution $\boldsymbol{\theta}^{\star, s}$ that leads to the lowest indicator $\operatorname{det}\left(\left[\pi\left(\hat{\mathbf{n}}\left(\boldsymbol{\theta}^{\star, s}\right)\right]-\right.\right.$ $[\chi]-[H])$ is used to determine if ellipticity is lost at the considered Gauss point. Using the solution for the normal vector, $\mathbf{n}^{\star}$, the determinant of the acoustic tensor can be obtained using the relations

$$
\operatorname{det}\left(\mathbf{Q}_{e p}\left(\mathbf{n}^{\star}\right)\right)=\operatorname{det}\left(\mathbf{Q}_{e l}\left(\mathbf{n}^{\star}\right)\right) \operatorname{det}\left(\overline{\mathbf{Q}}_{e p}\left(\mathbf{n}^{\star}\right)\right), \quad \operatorname{det}\left(\overline{\mathbf{Q}}_{e p}\left(\mathbf{n}^{\star}\right)\right)=\operatorname{det}\left([1]-[G]^{-1}\left[\pi\left(\mathbf{n}^{\star}\right)\right]\right)
$$

with $\operatorname{det}\left(\mathbf{Q}_{e l}(\mathbf{n})\right)=(\lambda+2 \mu) \mu^{2}$ for any normal vector $\mathbf{n}$. It should be noted that this analysis relies on the tangent elasto-plastic tensor $\mathbb{C}^{e p}$ in the strong formulation of the boundary value problem (see Eqs. (22) and (23)). In the case of numerical simulations involving the finite element method and an implicit discretization scheme, the classical loss of ellipticity 
analysis of the discretized boundary value problem can also be achieved by considering the algorithmic acoustic tensor (see, e.g. [46]). The numerical procedures presented by [8] and [9] can be used for loss of ellipticity analysis of the continuum and discretized boundary value problems.

\section{Family of linear yield criteria in principal stress space}

In this work, we focus on a family of yield functions first introduced by [47] as a family of generalized Mohr-Coulomb criteria, i.e., a yield function that is a linear combination of the principal stresses. For instance, the Tresca yield function which assumes that yielding takes place when the shear stress reaches a maximum value, belongs to such a family. The twin shear stress model of [37] and the more recent mean influence factor model of [38] also consider yield functions that depend linearly on the principal stresses. The maximum and minimum eigenvalues, $\sigma_{1}$ and $\sigma_{3}$, are defined as

$$
\sigma_{1}(\boldsymbol{\sigma})=\max \left\{\langle\mathbf{z}, \boldsymbol{\sigma} \mathbf{z}\rangle \mid \mathbf{z} \in \mathbb{R}^{3},\|\mathbf{z}\|=1\right\}, \quad \sigma_{3}(\boldsymbol{\sigma})=\min \left\{\langle\mathbf{z}, \boldsymbol{\sigma} \mathbf{z}\rangle \mid \mathbf{z} \in \mathbb{R}^{3},\|\mathbf{z}\|=1\right\}
$$

The intermediate eigenvalue $\sigma_{2}(\boldsymbol{\sigma})$ is given by $\sigma_{2}(\boldsymbol{\sigma})=\operatorname{Tr}(\boldsymbol{\sigma})-\sigma_{1}(\boldsymbol{\sigma})-\sigma_{3}(\boldsymbol{\sigma})$ and hence $\sigma_{1}(\boldsymbol{\sigma}) \geq \sigma_{2}(\boldsymbol{\sigma}) \geq \sigma_{3}(\boldsymbol{\sigma})$. By invoking the spectral theorem, the Cauchy stress tensor admits the decomposition

$$
\boldsymbol{\sigma}=\sigma_{1}(\boldsymbol{\sigma}) \mathbf{m}_{1}(\boldsymbol{\sigma}) \otimes \mathbf{m}_{1}(\boldsymbol{\sigma})+\sigma_{2}(\boldsymbol{\sigma}) \mathbf{m}_{2}(\boldsymbol{\sigma}) \otimes \mathbf{m}_{2}(\boldsymbol{\sigma})+\sigma_{3}(\boldsymbol{\sigma}) \mathbf{m}_{3}(\boldsymbol{\sigma}) \otimes \mathbf{m}_{3}(\boldsymbol{\sigma}),
$$

where $\mathbf{m}_{1}, \mathbf{m}_{2}$, and $\mathbf{m}_{3}$ are the eigenvectors associated to the ordered principal stresses. When no confusion can be made, the dependence of the principal stresses and principal directions on the stress tensor will be dropped. We further introduce a vector-valued parameter $\mathbf{a}=\left(a_{1}, a_{2}, a_{3}\right)$ that takes its values in a subset $\mathcal{C}$ of $\mathbb{R}^{3}$ to be defined, and such that

$$
\sum_{i=1}^{3} a_{i}=0 .
$$

We consider the following yield function that is linear in the principal stresses:

$$
\phi(\boldsymbol{\sigma}, R)=\langle\mathbf{a}, \hat{\boldsymbol{\sigma}}(\boldsymbol{\sigma})\rangle-R,
$$


where $\hat{\boldsymbol{\sigma}}=\left(\sigma_{1}, \sigma_{2}, \sigma_{3}\right)$ is the vector gathering the ordered eigenvalues of $\boldsymbol{\sigma}$. Under the assumptions given by Eq. 67), it can readily be deduced that $\phi$ is pressure insensitive, $\phi(\boldsymbol{\sigma}+q \mathbf{1})=\phi(\boldsymbol{\sigma})$ for any $q \in \mathbb{R}$. Due to this property, the yield function depends on only two independent parameters. In addition, it should be noted that the parameter $\mathbf{a}=\left(a_{1}, a_{2}, a_{3}\right)$ takes its values in a subset $\mathcal{C}_{\text {conv }}$ of $\mathbb{R}^{3}$ that ensures the convexity of the yield function. The functions $\boldsymbol{\sigma} \mapsto \sigma_{1}(\boldsymbol{\sigma})$ and $\boldsymbol{\sigma} \mapsto\left(-\sigma_{3}(\boldsymbol{\sigma})\right)$ are both convex but the intermediate principal stress $\boldsymbol{\sigma} \mapsto \sigma_{2}(\boldsymbol{\sigma})$ is neither convex nor concave. Hence, in the following we shall restrict ourselves to an admissible set of the form $\mathcal{C}=\mathcal{C}_{\text {conv }} \cap \mathcal{C}_{\text {inc }}$, where

$$
\mathcal{C}_{\text {conv }}=\left\{\mathbf{a} \in \mathbb{R}^{3} \mid a_{1}>a_{2}>a_{3}, a_{1}>0, a_{3}<0\right\}, \quad \mathcal{C}_{\text {inc }}=\left\{\mathbf{a} \in \mathbb{R}^{3} \mid a_{1}+a_{2}+a_{3}=0\right\}
$$

for the parameter a. For simplicity, three particular cases will be highlighted in the sequel of this paper, namely,

(C1) Case of the Tresca model obtained for $a_{1}=1, a_{2}=0$, and $a_{3}=-1$, that is

$$
\phi^{(0)}(\boldsymbol{\sigma}, R) \equiv \sigma_{1}(\boldsymbol{\sigma})-\sigma_{3}(\boldsymbol{\sigma})-R
$$

(C2) Case of a modified Tresca model obtained for $a_{1}=1, a_{2}=-\delta, a_{3}=-1+\delta$, i.e.,

$$
\phi^{(\delta)}(\boldsymbol{\sigma}, R) \equiv \sigma_{1}(\boldsymbol{\sigma})-\delta \sigma_{2}(\boldsymbol{\sigma})-(1-\delta) \sigma_{3}(\boldsymbol{\sigma})-R
$$

with $-1<\delta<1 / 2$. This model will be referred to as the $\delta$-Tresca model.

(C3) Case of a modified Tresca model obtained for $a_{1}=1-\tau, a_{2}=\tau$, and $a_{3}=-1$, yielding

$$
\phi^{(\tau)}(\boldsymbol{\sigma}, R) \equiv(1-\tau) \sigma_{1}(\boldsymbol{\sigma})+\tau \sigma_{2}(\boldsymbol{\sigma})-\sigma_{3}(\boldsymbol{\sigma})-R
$$

with $-1<\tau<1 / 2$. This model will be referred to as the $\tau$-Tresca model.

These models are illustrated by sketches of the elastic domain in Fig. 1 for different values of $\delta$ and $\tau$. It can be seen that the parameter $\delta$ (resp. $\tau$ ) allows for displacing the left corner (resp. right corner) along the hexagone's axes. A combination of these parameterizations results in simultaneous displacements of both corners. It should be noted that given the 

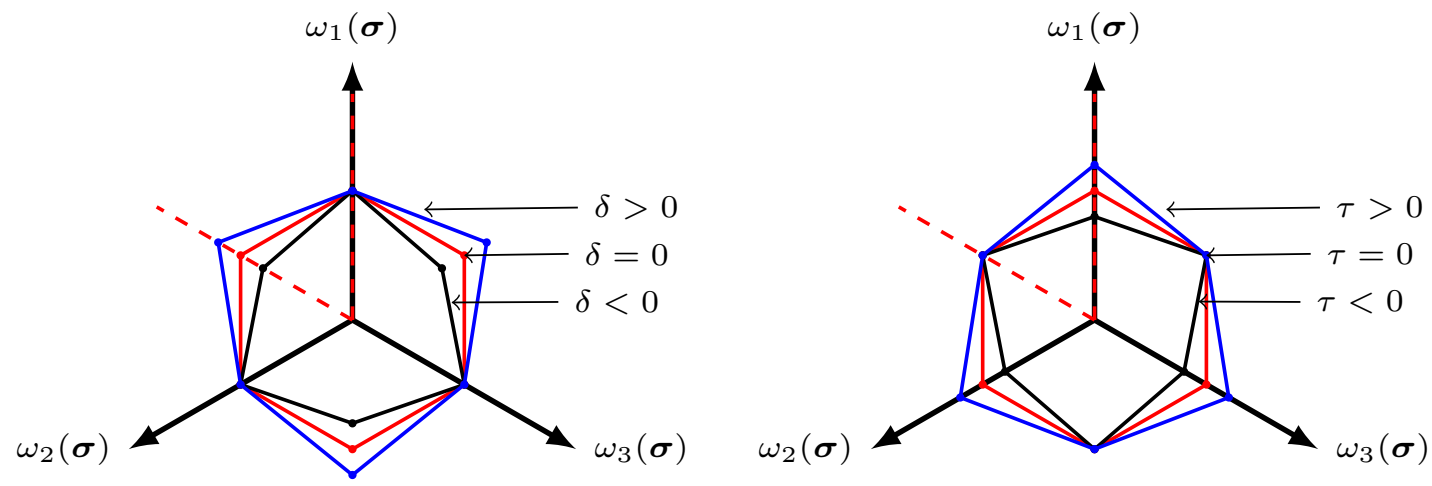

Figure 1: Elastic domains in the deviatoric plane for different values of the parameters $\delta$ and $\tau$. The classical Tresca model (in red) is recovered for $\delta=0$ (or $\tau=0$ ) while $\delta \neq 0$ and $\tau \neq 0$ modify the positions of the left and right corners along the hexagone's axes. Here, $\omega_{1}(\boldsymbol{\sigma}), \omega_{2}(\boldsymbol{\sigma}), \omega_{3}(\boldsymbol{\sigma})$ denote the eigenvalues of the stress tensor $\boldsymbol{\sigma}$ without particular ordering.

assumption of ordered principal stresses, the yield functions $\phi^{(\delta)}$ and $\phi^{(\tau)}$ are distinct and only the first sextants of the elastic domains depicted in Fig.1 1 are relevant. The twin shear stress model [37] can also be recovered by considering the yield function $\phi^{(\delta)}$ with $\delta=1 / 2$ (resp. $\phi^{(\tau)}$ with $\left.\tau=1 / 2\right)$ as the yield function if $\sigma_{2} \leq\left(\sigma_{1}+\sigma_{3}\right) / 2\left(\operatorname{resp}\right.$. if $\left.\sigma_{2} \geq\left(\sigma_{1}+\sigma_{3}\right) / 2\right)$, that is to say,

$$
\phi^{(T S S)}= \begin{cases}\sigma_{1}-\frac{1}{2}\left(\sigma_{2}+\sigma_{3}\right)-R, & \sigma_{2} \leq \frac{1}{2}\left(\sigma_{1}+\sigma_{3}\right), \\ \frac{1}{2}\left(\sigma_{1}+\sigma_{2}\right)-\sigma_{3}-R, & \sigma_{2} \geq \frac{1}{2}\left(\sigma_{1}+\sigma_{3}\right) .\end{cases}
$$

Similarly, the recent mean influence factor model [38] can be recovered by taking $\delta=(1-$ $2 \sqrt{3}+\sqrt{13}) / 4$ and $\tau=(1-2 \sqrt{3}+\sqrt{13}) / 4$.

\subsection{Flow rule associated to the family of linear yield functions in principal stress space}

In this section, we briefly determine the flow rule associated to the yield function defined by Eq. (68) for arbitrary values of the parameter a. Given that the mapping $g \mapsto \phi(\cdot, R)$ is continuously differentiable, the flow rule $(9)$ reduces to

$$
\dot{\boldsymbol{\varepsilon}}^{p} \in \dot{\gamma} \partial \phi_{\boldsymbol{\sigma}}(\boldsymbol{\sigma}, R), \quad \dot{p}=\dot{\gamma} \frac{\partial \phi(\boldsymbol{\sigma}, R)}{\partial R},
$$




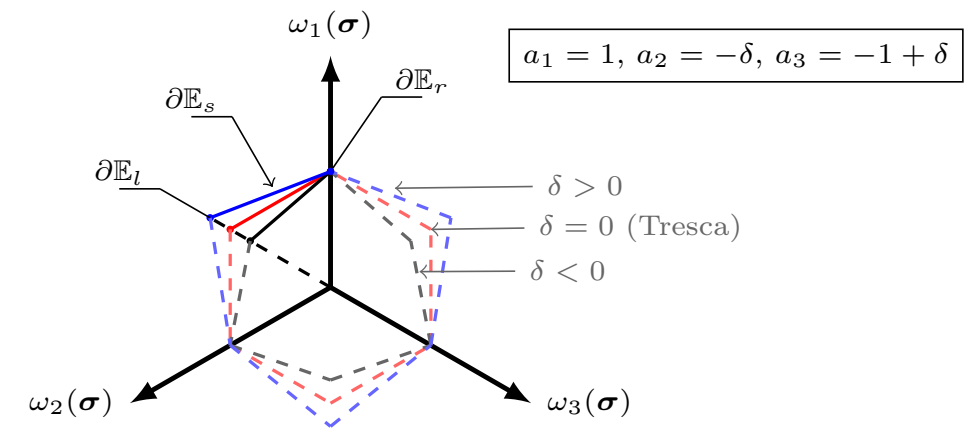

Figure 2: Elastic domains in the deviatoric plane for different values of the parameter $\delta$ together with the the subsets $\partial \mathbb{E}_{l}, \partial \mathbb{E}_{s}$, and $\partial \mathbb{E}_{r}$. Here, $\omega_{1}(\boldsymbol{\sigma}), \omega_{2}(\boldsymbol{\sigma}), \omega_{3}(\boldsymbol{\sigma})$ denote the eigenvalues of the stress tensor $\boldsymbol{\sigma}$ without particular ordering.

where $\partial_{\boldsymbol{\sigma}} \phi$ is the subdifferential of the yield function $\phi$ for a fixed yield stress $R$, i.e.,

$$
\partial \phi_{\boldsymbol{\sigma}}(\boldsymbol{\sigma}, R)=\{\hat{\mathbf{p}} \in \mathcal{P} \mid \phi(\tilde{\boldsymbol{\sigma}}, R) \geq \phi(\boldsymbol{\sigma}, R)+\hat{\mathbf{p}}:(\tilde{\boldsymbol{\sigma}}-\boldsymbol{\sigma}), \forall \tilde{\boldsymbol{\sigma}} \in \mathcal{S}\}
$$

The yield function $\phi$ is differentiable whenever the principal stresses are distinct, i.e., $\sigma_{1}(\boldsymbol{\sigma})>\sigma_{2}(\boldsymbol{\sigma})>\sigma_{3}(\boldsymbol{\sigma})$, while it is subdifferentiable whenever $\sigma_{1}(\boldsymbol{\sigma})=\sigma_{2}(\boldsymbol{\sigma}) \geq \sigma_{3}(\boldsymbol{\sigma})$ or $\sigma_{1}(\boldsymbol{\sigma}) \geq \sigma_{2}(\boldsymbol{\sigma})=\sigma_{3}(\boldsymbol{\sigma})$. For further developments, we introduce the following subsets of generalized stresses $(\boldsymbol{\sigma}, R)$. The boundary of the elastic domain is denoted by $\partial \mathbb{E}$ and decomposed as a union of disjoint sets $\partial \mathbb{E}=\partial \mathbb{E}_{l} \cup \partial \mathbb{E}_{s} \cup \partial \mathbb{E}_{r}$. These sets correspond to the left corner, smooth portion, and right corner of the yield surface, that is

$$
\begin{aligned}
& \partial \mathbb{E}_{l}=\left\{(\boldsymbol{\sigma}, R) \in \partial \mathbb{E} \mid \sigma_{1}(\boldsymbol{\sigma})-\sigma_{2}(\boldsymbol{\sigma})=0, \sigma_{2}(\boldsymbol{\sigma}) \geq \sigma_{3}(\boldsymbol{\sigma})\right\} \\
& \partial \mathbb{E}_{s}=\left\{(\boldsymbol{\sigma}, R) \in \partial \mathbb{E} \mid \sigma_{1}(\boldsymbol{\sigma})>\sigma_{2}(\boldsymbol{\sigma})>\sigma_{3}(\boldsymbol{\sigma})\right\} \\
& \partial \mathbb{E}_{r}=\left\{(\boldsymbol{\sigma}, R) \in \partial \mathbb{E} \mid \sigma_{1}(\boldsymbol{\sigma}) \geq \sigma_{2}(\boldsymbol{\sigma}), \sigma_{2}(\boldsymbol{\sigma})-\sigma_{3}(\boldsymbol{\sigma})=0\right\}
\end{aligned}
$$

The closure of the elastic domain being denoted by $\mathbb{E}$, we let $\mathbb{E}_{0}$ be its open counterpart. The subsets given by Eq. (76) are illustrated for the $\delta$-Tresca model in Fig. 2. These definitions hold for any vector-valued parameter $\mathbf{a} \in \mathcal{C}$ and thus for the three particular cases defined by Eqs. (70)-(72). An explicit expression of the subdifferential set $\partial_{\sigma} \phi(75)$ is reported in Appendix Appendix A. Such an expression extends existing results for the Tresca and MohrCoulomb criteria obtained by [39] by [40], respectively. In addition, it can be used to derive 
an implicit integration scheme but this is out of scope of this paper and left for further work. Here, we focus on the multisurface form of flow rule in order to use the loss of ellipticity conditions summarized in section 3. For this purpose, it can be noticed that the elastic domain can also be defined in terms of the following three yield functions

$\phi_{1}(\boldsymbol{\sigma}, R)=\langle\hat{\boldsymbol{\sigma}}, \mathbf{a}\rangle-R, \quad \phi_{2}(\boldsymbol{\sigma}, R)=a_{1} \sigma_{2}+a_{2} \sigma_{1}+a_{3} \sigma_{3}-R, \quad \phi_{3}(\boldsymbol{\sigma}, R)=a_{1} \sigma_{1}+a_{2} \sigma_{3}+a_{3} \sigma_{2}-R$.

Please note that this multisurface representation depends on a single yield stress $R$ and that the extension to distinct yield stresses could be considered in a future work. We propose to deduce the multisurface form of the flow rule $\dot{\boldsymbol{\varepsilon}}^{p}=\sum_{j=1}^{3} \dot{\gamma}_{j} \mathbf{N}_{j}(\boldsymbol{\sigma})$ (see Eq. 16) from the subdifferential-based flow rule $\dot{\boldsymbol{\varepsilon}}^{p} \in \dot{\gamma} \partial_{\boldsymbol{\sigma}} \phi(\boldsymbol{\sigma})$. In contrast with the abstract flow rule, the Lagrange multipliers $\dot{\gamma}_{1}, \dot{\gamma}_{2}, \dot{\gamma}_{3}$ can be eliminated in order to derive the rate form of the stressstrain relation. Let then $\mu_{1}, \mu_{2}, \mu_{3}$ be three positive real numbers such that $\mu_{1}+\mu_{2}+\mu_{3}=1$, and let $\tilde{\phi}$ be the auxiliary yield function defined as

$$
\tilde{\phi}(\boldsymbol{\sigma}, R)=\mu_{1} \phi_{1}(\boldsymbol{\sigma}, R)+\mu_{2} \phi_{2}(\boldsymbol{\sigma}, R)+\mu_{3} \phi_{3}(\boldsymbol{\sigma}, R) .
$$

In the sequel of this section, we shall denote by $\mathbf{N}^{j}$ the second-order tensors defined as $\mathbf{N}^{j}=\nabla_{\boldsymbol{\sigma}} \phi_{j}$, that is

$$
\begin{aligned}
& \mathbf{N}^{1}=\sum_{i=1}^{3} a_{i} \mathbf{m}_{i} \otimes \mathbf{m}_{i}, \quad \mathbf{N}^{2}=a_{1} \mathbf{m}_{2} \otimes \mathbf{m}_{2}+a_{2} \mathbf{m}_{1} \otimes \mathbf{m}_{1}+a_{3} \mathbf{m}_{3} \otimes \mathbf{m}_{3}, \\
& \mathbf{N}^{3}=a_{1} \mathbf{m}_{1} \otimes \mathbf{m}_{1}+a_{2} \mathbf{m}_{3} \otimes \mathbf{m}_{3}+a_{3} \mathbf{m}_{2} \otimes \mathbf{m}_{2} .
\end{aligned}
$$

In order to determine possible sets of values for $\mu_{1}, \mu_{2}, \mu_{3}$ such that $\tilde{\phi}(\boldsymbol{\sigma}, R)=\phi(\boldsymbol{\sigma}, R)$, four cases have to be distinguished depending on the current values of the principal stresses:

(a) If $(\boldsymbol{\sigma}, R) \in \mathbb{E}_{0}$ then clearly $\tilde{\phi}(\boldsymbol{\sigma})<0$ regardless of the values of $\mu_{1}, \mu_{2}$, and $\mu_{3}$. Upon injecting Eq. (77) into Eq. (78), it is found that $\tilde{\phi}(\boldsymbol{\sigma})=\phi(\boldsymbol{\sigma})$ for any $\left(\mu_{1}, \mu_{2}, \mu_{3}\right)$ such that $\mu_{1}+\mu_{2}=1$ and $\mu_{2}=\mu_{3}$.

(b) Consider the case for which $(\boldsymbol{\sigma}, R) \in \partial \mathbb{E}_{s}$, then $\phi_{1}(\boldsymbol{\sigma}, R)=0$. Hence, it can be deduced that $\phi(\boldsymbol{\sigma}, R)=\tilde{\phi}(\boldsymbol{\sigma}, R)$ for any $\mu_{1} \geq 0$ and $\mu_{2}=0, \mu_{3}=0$. It follows that the 
subdifferential set $\partial_{\boldsymbol{\sigma}} \phi$ is given by

$$
\partial \phi_{\boldsymbol{\sigma}}(\boldsymbol{\sigma}, R)=\mu_{1} \partial_{\boldsymbol{\sigma}} \phi_{1}(\boldsymbol{\sigma}, R)
$$

and thus $\dot{\boldsymbol{\varepsilon}}^{p}=\mu_{1} \dot{\gamma} \partial_{\boldsymbol{\sigma}} \phi_{1}(\boldsymbol{\sigma})$. Given that the mapping $\boldsymbol{\sigma} \mapsto \phi_{1}(\boldsymbol{\sigma}, \cdot)$ is differentiable, the set $\partial_{\boldsymbol{\sigma}} \phi_{1}(\boldsymbol{\sigma})$ reduces to the singleton $\left\{\mathbf{N}_{1}\right\}$. Based on this result, the flow rule takes the form

$$
\dot{\varepsilon}^{p}=\dot{\gamma}_{1} \mathbf{N}_{1}, \quad \dot{\gamma}_{1} \equiv \dot{\gamma},
$$

where the property $\mu_{1}=1$ has been used.

(c) Let $(\boldsymbol{\sigma}, R) \in \partial \mathbb{E}_{l}$, then the generalized stresses satisfy the conditions $\phi_{1}(\boldsymbol{\sigma}, R)=0$ and $\phi_{2}(\boldsymbol{\sigma}, R)=0$. It follows that $\phi(\boldsymbol{\sigma}, R)=\tilde{\phi}(\boldsymbol{\sigma}, R)$ for any $\mu_{1} \geq 0, \mu_{2} \geq 0$, and $\mu_{3}=0$. In this case, the subdifferential set $\partial_{\sigma} \phi$ can also be written as

$$
\partial_{\boldsymbol{\sigma}} \phi(\boldsymbol{\sigma}, R)=\mu_{1} \partial_{\boldsymbol{\sigma}} \phi_{1}(\boldsymbol{\sigma}, R)+\mu_{2} \partial_{\boldsymbol{\sigma}} \phi_{2}(\boldsymbol{\sigma}, R)
$$

Hence, it can be deduced that flow rule takes the form

$$
\dot{\varepsilon}^{p}=\dot{\gamma}_{1} \mathbf{N}_{1}+\dot{\gamma}_{2} \mathbf{N}_{2}, \quad \dot{\gamma}_{1} \equiv \mu_{1} \dot{\gamma}, \quad \dot{\gamma}_{2} \equiv \mu_{2} \dot{\gamma}
$$

(d) Finally, consider the case for which $(\boldsymbol{\sigma}, R) \in \partial \mathbb{E}_{r}$. This condition is equivalent to $\phi_{1}(\boldsymbol{\sigma}, R)=0$ and $\phi_{3}(\boldsymbol{\sigma}, R)=0$. Hence, $\tilde{\phi}(\boldsymbol{\sigma}, R)=\mu_{2} \phi_{2}(\boldsymbol{\sigma}, R)$ for any positive $\mu_{1} \geq 0$ and $\mu_{3} \geq 0$. It follows that if $\mu_{2}=0$, then $\phi(\boldsymbol{\sigma}, R)=\tilde{\phi}(\boldsymbol{\sigma}, R)$. The subdifferential set $\partial_{\boldsymbol{\sigma}} \phi$ can be additively decomposed as

$$
\partial_{\boldsymbol{\sigma}} \phi(\boldsymbol{\sigma}, R)=\mu_{1} \partial_{\boldsymbol{\sigma}} \phi_{1}(\boldsymbol{\sigma}, R)+\mu_{3} \partial_{\boldsymbol{\sigma}} \phi_{3}(\boldsymbol{\sigma}, R),
$$

where $\partial_{\boldsymbol{\sigma}} \phi_{3}=\left\{\mathbf{N}_{3}\right\}$. The flow rule $\boldsymbol{\varepsilon}^{p} \in \dot{\gamma} \partial_{\boldsymbol{\sigma}} \phi$ takes the form

$$
\dot{\varepsilon}^{p}=\dot{\gamma}_{1} \mathbf{N}_{1}+\dot{\gamma}_{3} \mathbf{N}_{3}, \quad \dot{\gamma}_{1} \equiv \mu_{1} \dot{\gamma}, \quad \dot{\gamma}_{3} \equiv \mu_{3} \dot{\gamma}
$$

As a result, it is always possible to find a triplet $\left(\mu_{1}, \mu_{2}, \mu_{3}\right)$ such that $\phi(\boldsymbol{\sigma})=\sum_{j=1}^{3} \mu_{j} \phi_{j}(\boldsymbol{\sigma})$ for all $\sigma \in \mathbb{M}_{S}^{3}$. It can also be seen that this result leads to an equivalence between the 
abstract flow rule given by Eq. (9) and the multisurface flow rule given by Eq. (16). One advantage of the subdifferential-based flow rule is that it can lead to a simpler fully implicit integration scheme as shown by [36] for Mohr-Coulomb plasticity. It should be emphasized that for $a_{1}=1, a_{2}=0$, and $a_{3}=-1$, the multisurface and subdifferential flow rule of the Tresca yield function are recovered (see, e.g., [29] and [39]).

\subsection{Critical hardening moduli}

With the yield function and flow rule now clearly defined, we are able to analyze under which circumstances loss of ellipticity may occur. In the sequel of this section, Young's modulus is set to $E=210 \mathrm{GPa}$ and unless stated otherwise, the Poisson's ratio is chosen as $\nu=0.3$. The aim of this section is to determine the critical hardening moduli and the associated normals vector $\mathbf{n}$ to the shear bands. The hardening matrix $[H]$ is assumed to belong to the family defined by Eq. (44) with $\ell=1$. Given that the yield function (77) can be represented by three mechanisms (see Eq. (68)), the hardening matrix simply takes the form

$$
[H]=H\left[i_{3}\right], \quad\left[i_{3}\right]=\left[\begin{array}{lll}
1 & 1 & 1 \\
1 & 1 & 1 \\
1 & 1 & 1
\end{array}\right] .
$$

Three different situations will be considered, namely, a current stress state that lies on the smooth portion, left corner, right corner of the yield surface. For each situation, the three particular cases described in section 4 (see Fig. 1) will be illustrated. The critical hardening moduli and normals to shear bands are denoted by

- $H_{0, s}^{c r i t}, H_{0, l}^{c r i t}, H_{0, r}^{c r i t}$ and $\mathbf{n}_{0, s}^{\text {crit }}, \mathbf{n}_{0, l}^{\text {crit }}, \mathbf{n}_{0, r}^{\text {crit }}$ for the Tresca model,

- $H_{\delta, s}^{c r i t}, H_{\delta, l}^{c r i t}, H_{\delta, r}^{c r i t}$ and $\mathbf{n}_{\delta, s}^{c r i t}, \mathbf{n}_{\delta, l}^{\text {crit }}, \mathbf{n}_{\delta, r}^{\text {crit }}$ for the $\delta$-Tresca model,

- $H_{\tau, s}^{c r i t}, H_{\tau, l}^{c r i t}, H_{\tau, r}^{c r i t}$ and $\mathbf{n}_{\tau, s}^{c r i t}, \mathbf{n}_{\tau, l}^{c r i t}, \mathbf{n}_{\tau, r}^{c r i t}$ for the $\tau$-Tresca model.

The case of the smooth portion of the yield surface is first studied in section 4.2.1. The left and right corners are then addressed in section 4.2 .2 . 


\subsubsection{Critical hardening modulus on the smooth portion of the yield surfaces}

If the current stress state lies on the smooth portion of the yield surface, $(\boldsymbol{\sigma}, R) \in \partial \mathbb{E}_{S}$, the critical hardening modulus can be obtained using the results presented in section 3.1 . Let $k^{\star}$ be the index defined such that $\left|a_{k^{\star}}\right|=\min \left\{\left|a_{1}\right|,\left|a_{2}\right|,\left|a_{3}\right|\right\}$. Using Eq. (32), the critical hardening modulus and the associated normal $\mathbf{n}$ are given by

$$
H_{s}^{c r i t}=-E a_{k^{\star}}^{2}, \quad n_{k^{\star}}=0, \quad n_{i}^{2}=\frac{a_{i}+\nu a_{k^{\star}}}{a_{i}-a_{j}}, \quad n_{j}^{2}=1-n_{i}^{2}, \quad i, j \neq k^{\star} .
$$

If $n_{i}=1$ and $n_{j}=n_{k}=0$ for any $i \in\{1,2,3\}$, then the critical modulus is given by Eq. (34). However, it has been found that this solution yields less favorable critical hardening moduli.

(C1) In the particular case of a Tresca model, that is $a_{1}=1, a_{2}=0$, and $a_{3}=-1$, the most favorable critical hardening modulus $H_{0, s}^{\text {crit }}$ and the normal vector $\mathbf{n}_{0, s}^{\text {crit }}$ are given by

$$
H_{0, s}^{c r i t}=0, \quad \mathbf{n}_{0, s}^{c r i t}=\frac{1}{\sqrt{2}}\left[\begin{array}{c} 
\pm 1 \\
0 \\
\pm 1
\end{array}\right]_{\left(\mathbf{m}_{i}\right)_{i=1}^{3}}
$$

meaning that the shear band is oriented with a $45^{\circ}$ angle in the plane spanned by $\left(\mathbf{m}_{1}, \mathbf{m}_{3}\right)$.

(C2) In the case of the $\delta$-Tresca model, it is found that $a_{k^{\star}}=a_{2}$ and thus $a_{k^{\star}}=-\delta$ for any $\delta \in]-1,1 / 2\left[\right.$. The first possible critical hardening modulus is given by $H_{\delta, s}^{c r i t}=-E \delta^{2}$ and the associated normal vector $\mathbf{n}_{\delta, s}^{\text {crit }}$ can be written as $\mathbf{n}_{\delta, s}^{\text {crit }}=(\cos (\theta), 0, \sin (\theta))$ in the principal stress basis. In summary, the following solutions are found for the critical hardening modulus and the normal vector:

$$
\begin{aligned}
& H_{\delta, s}^{c r i t}=-E \delta^{2}, \quad \mathbf{n}_{\delta, s}^{\text {crit }}=\frac{1}{\sqrt{2-\delta}} {\left[\begin{array}{c} 
\pm \sqrt{1-\nu \delta} \\
0 \\
\pm \sqrt{1-\delta(1-\nu)}
\end{array}\right]_{\left(\mathbf{m}_{i}\right)_{i=1}^{3}} } \\
& \text { or } \quad \mathbf{n}_{\delta, s}^{c r i t}=\frac{1}{\sqrt{2-\delta}}\left[\begin{array}{c} 
\pm \sqrt{1-\delta(1-\nu)} \\
0 \\
\pm \sqrt{1-\nu \delta}
\end{array}\right]_{\left(\mathbf{m}_{i}\right)_{i=1}^{3}} .
\end{aligned}
$$



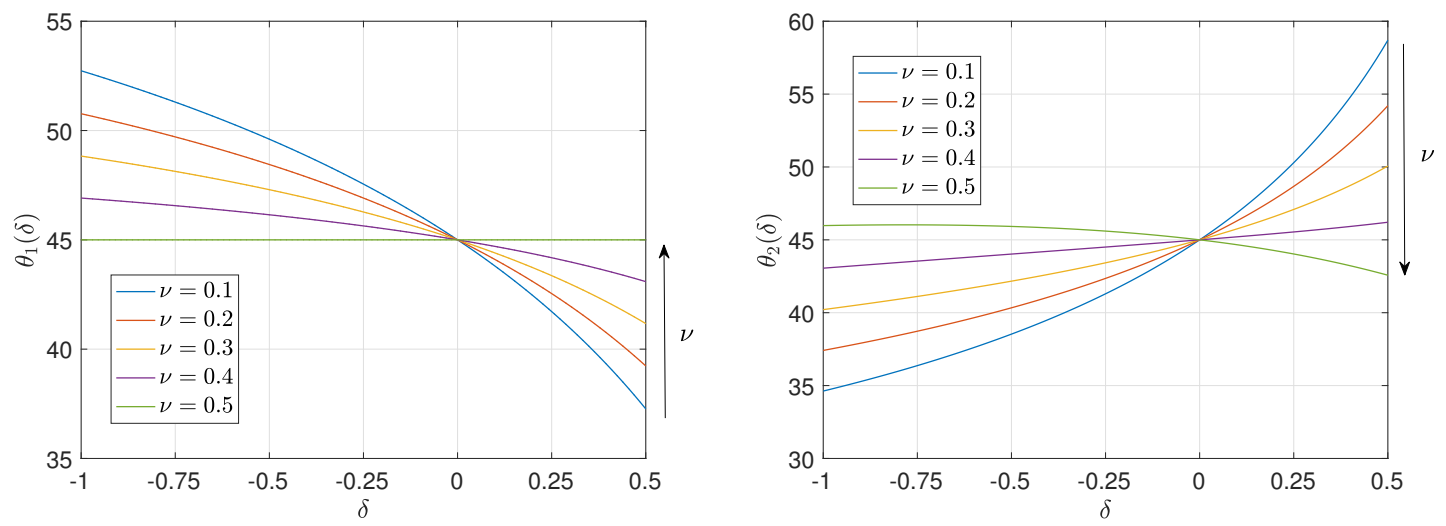

Figure 3: Graphs of the angles $\delta \mapsto \theta_{1}(\delta)$ and $\delta \mapsto \theta_{2}(\delta)$ formed by the two possible normal vectors to the shear band on the smooth portion of the yield surface, and depending on the value of the Poisson ratio.

Graphs of the mappings $\delta \mapsto \theta_{1}(\delta)$ and $\delta \mapsto \theta_{2}(\delta)$ associated to the above normal vectors are shown in Fig. 3 for selected values of Poisson's ratio $\nu \in[0.1,0.5]$. It can easily be verified that $1-\nu \delta>0$ and $1-\delta(1-\nu)>0$ for any $\nu \in[0,0.5]$ and $\delta \in]-1,1 / 2[$.

(C3) In the case of the $\tau$-Tresca model with $a_{1}=1-\tau, a_{2}=\tau, a_{3}=-1$, one has $a_{k^{\star}}=\tau$. Similarly to Eq. 89 , it follows that the critical hardening modulus $H_{\tau, s}^{\text {crit }}$ and the possible normal vectors take the forms

$$
\begin{aligned}
& H_{\tau, s}^{c r i t}=-E \tau^{2}, \quad \mathbf{n}_{\tau, s}^{c r i t} \frac{1}{\sqrt{2-\tau}}= {\left[\begin{array}{c} 
\pm \sqrt{1-\tau(1-\nu)} \\
0 \\
\pm \sqrt{1-\nu \tau}
\end{array}\right]_{\left(\mathbf{m}_{i}\right)_{i=1}^{3}} } \\
& \text { or } \quad \mathbf{n}_{\tau, s}^{c r i t}=\frac{1}{\sqrt{2-\tau}}\left[\begin{array}{c} 
\pm \sqrt{1-\nu \tau} \\
0 \\
\pm \sqrt{1-\tau(1-\nu)}
\end{array}\right]_{\left(\mathbf{m}_{i}\right)_{i=1}^{3}} .
\end{aligned}
$$

Similar graphs of the associated angles $\theta_{1}$ and $\theta_{2}$ are obtained as those given by the right and left panel of Fig. 3, respectively. From the previous developments, it follows that $1-\nu \tau>0$ and $1-\tau(1-\nu)>0$ for any $\nu \in[0,0.5]$ and $\tau \in]-1,1 / 2[$.

As a result, it can be deduced that the standard Tresca model leads to the largest critical 
hardening modulus whenever the stress tensor lies on the smooth portion of the yield surface. In the next section, the same analysis is performed on the corners of the yield surface.

\subsubsection{Critical hardening modulus on the corners of the Tresca yield surface}

If the current stress state lies on a corner of the yield surface, then the critical hardening modulus can be obtained using the developments of section 3.2. In the following, we first assume that the stress state lies on the right corner, that is $(\boldsymbol{\sigma}, R) \in \partial \mathbb{E}_{r}$ (see Eq. (76) and Fig. 2). The hardening matrices $\left[H_{0, r}\right],\left[H_{\delta, r}\right]$, and $\left[H_{\tau, r}\right]$ denote the restriction of $[H]$ when the stresses lie on the right corner of the Tresca model and its variants, that is

$$
\left[H_{0, r}\right]=H_{0, r}\left[i_{2}\right], \quad\left[H_{\delta, r}\right]=H_{\delta, r}\left[i_{2}\right], \quad\left[H_{\tau, r}\right]=H_{\tau, r}\left[i_{2}\right], \quad\left[i_{2}\right]=\left[\begin{array}{ll}
1 & 1 \\
1 & 1
\end{array}\right]
$$

and we let $\left[\pi_{r}^{(0)}(\mathbf{n})\right],\left[\pi_{r}^{(\delta)}(\mathbf{n})\right]$, and $\left[\pi_{r}^{(\tau)}(\mathbf{n})\right]$ be the matrix given by Eq. (38) on the right corner of the Tresca, $\delta$-Tresca, and $\tau$-Tresca models, respectively. Similar notations are used for the left corners.

As a first illustration, we consider the case of the standard Tresca model on the right corner of the yield surface. The set of active mechanisms is thus given by $\mathbb{J}=\{1,3\}$. The eigenvalues of $\mathbf{N}^{1}$ and $\mathbf{N}^{3}$ are $N_{1}^{1}=a_{1}, N_{2}^{1}=a_{2}, N_{3}^{1}=a_{3}$, and $N_{1}^{3}=a_{1}, N_{2}^{3}=a_{3}, N_{3}^{3}=a_{2}$. The matrix $\left[\pi_{r}^{(0)}(\mathbf{n})\right]$ takes the form

$$
\left[\pi_{r}^{(0)}(\mathbf{n})\right]=4 \mu\left[\begin{array}{cc}
\alpha\left(n_{1}^{2}-n_{3}^{2}\right)^{2}+n_{1}^{2}+n_{3}^{2} & \alpha\left(n_{1}^{2}-n_{3}^{2}\right)\left(n_{1}^{2}-n_{2}^{2}\right)+n_{1}^{2} \\
\alpha\left(n_{1}^{2}-n_{3}^{2}\right)\left(n_{1}^{2}-n_{2}^{2}\right)+n_{1}^{2} & \alpha\left(n_{1}^{2}-n_{2}^{2}\right)^{2}+n_{1}^{2}+n_{2}^{2}
\end{array}\right],
$$

where $\alpha=-(\lambda+\mu) /(\lambda+2 \mu)$ has been introduced for convenience. The critical hardening modulus $H_{0, r}^{\text {crit }}$ can be obtained using Eq. 46$)$ if the matrix $\left[\pi_{r}^{(0)}(\mathbf{n})\right]-[\chi]$ is invertible. It is found that the matrix $\left[\pi_{r}^{(0)}(\mathbf{n})\right]-[\chi]$ is singular whenever $n_{1}^{2}=n_{2}^{2}$ and $n_{3}=0, n_{1}^{2}=n_{3}^{2}$ and $n_{2}=0$ or $n_{1}=0$ and $n_{2}^{2}=n_{3}^{2}$. In these cases, the conditions for loss of ellipticity reduce to

$$
\begin{aligned}
& \operatorname{det}\left(\left[\pi_{r}^{(0)}(\mathbf{n})\right]-[\chi]-\left[H_{0, r}\right]\right)=H_{0, r} \mu \frac{3 \lambda+5 \mu}{\lambda+2 \mu}, \quad \text { if } \quad n_{1}^{2}=n_{2}^{2}=\frac{1}{2}, \quad n_{3}=0, \\
& \operatorname{det}\left(\left[\pi_{r}^{(0)}(\mathbf{n})\right]-[\chi]-\left[H_{0, r}\right]\right)=H_{0, r} \mu \frac{3 \lambda+5 \mu}{\lambda+2 \mu}, \quad \text { if } \quad n_{1}^{2}=n_{3}^{2}=\frac{1}{2}, \quad n_{2}=0, \\
& \operatorname{det}\left(\left[\pi_{r}^{(0)}(\mathbf{n})\right]-[\chi]-\left[H_{0, r}\right]\right)=0, \quad \text { if } \quad n_{2}^{2}=n_{3}^{2}=\frac{1}{2}, \quad n_{1}=0,
\end{aligned}
$$


and the hardening moduli solutions to $\operatorname{det}\left(\left[\pi_{r}^{(0)}(\mathbf{n})\right]-[\chi]-\left[H_{r}\right]\right)=0$ in Eqs. 93a - 93c are respectively given by $H_{0, r}^{(1)}(\mathbf{n})=0, H_{0, r}^{(2)}(\mathbf{n})=0$ and $H_{0, r}^{(3)}(\mathbf{n}) \in \mathbb{R}$. Whenever the matrix $\left[\pi_{r}^{(0)}(\mathbf{n})\right]-[\chi]$ is nonsingular, the hardening modulus $H_{0, r}: \mathbf{n} \mapsto H_{0, r}(\mathbf{n})$ satisfying $\operatorname{det}\left(\left[\pi_{r}^{(0)}(\mathbf{n})\right]-[\chi]-H_{r}\left[i_{2}\right]\right)=0$ is given by

$$
H_{0, r}(\mathbf{n})=\left(\left(\left[\pi_{r}^{(0)}(\mathbf{n})\right]-[\chi]\right)^{-1}:\left[i_{2}\right]\right)^{-1},
$$

and the associated critical value can be obtained by maximizing over the set of unit vectors $\mathbf{n}$ in $\mathbb{R}^{3}$ not including the three pathological cases given by Eqs. (93a)-(93c). A classical analytical approach would be to introduce a Lagrangian function together with a Lagrange multiplier associated to the normalization constraint $\|\mathbf{n}\|^{2}-1=0$. Unfortunately, even in this simple case, this strategy is intractable. Instead, the critical hardening modulus and the associated normal are computed numerically using the numerical procedure presented in section 3.3. Graphs of the hardening modulus with respect to some parameterized normal vectors are shown in Fig. 4 together with graphs of $\operatorname{det}\left(\mathbf{Q}_{e p}(\mathbf{n})\right)$ and $\operatorname{det}\left(\left[\pi_{r}^{(0)}(\mathbf{n})\right]-[\chi]-H_{r}\left[i_{2}\right]\right)$ with respect to $H_{r}$ in Fig.5. It is found that the conditions

$$
\operatorname{det}\left(\mathbf{Q}_{e p}\left(\mathbf{n}_{0, r}\right)\right)=0 \quad \text { and } \quad \operatorname{det}\left(\left[\pi_{r}^{(0)}\left(\mathbf{n}_{0, r}\right)\right]-[\chi]-H_{r}\left(\mathbf{n}_{0, r}\right)\left[i_{2}\right]\right)=0
$$

can be met as long as

$$
H_{0, r} \in \mathbb{R}, \quad \mathbf{n}_{0, r}=\left(0, n_{2}, n_{3}\right), \quad n_{2}^{2}+n_{3}^{2}=1
$$

This means that the acoustic tensor $\mathbf{Q}_{e p}$ is singular as soon as the material plastifies. In addition, the conditions given by Eq. (95) are also met for $H_{0, r}=0$ together with normal vectors $\mathbf{n}_{0, r}$ of the forms

$$
\mathbf{n}_{0, r}=\frac{1}{\sqrt{2}}\left[\begin{array}{c} 
\pm 1 \\
0 \\
\pm 1
\end{array}\right]_{\left(\mathbf{m}_{i}\right)_{i=1}^{3}} \quad \text { or } \quad \mathbf{n}_{0, r}=\frac{1}{\sqrt{2}}\left[\begin{array}{c} 
\pm 1 \\
\pm 1 \\
0
\end{array}\right]_{\left(\mathbf{m}_{i}\right)_{i=1}^{3}} .
$$

Furthermore, it can be seen in Figs. 4.5 that the conditions

$$
\left.\operatorname{det}\left(\mathbf{Q}_{e p}\left(\mathbf{n}_{0, r}\right)\right) \leq 0 \quad \text { and } \quad \operatorname{det}\left(\left[\pi_{r}^{(0)}\left(\mathbf{n}_{0, r}\right)\right]-[\chi]-H_{r}\left(\mathbf{n}_{0, r}\right)\left[i_{2}\right]\right)\right) \leq 0
$$




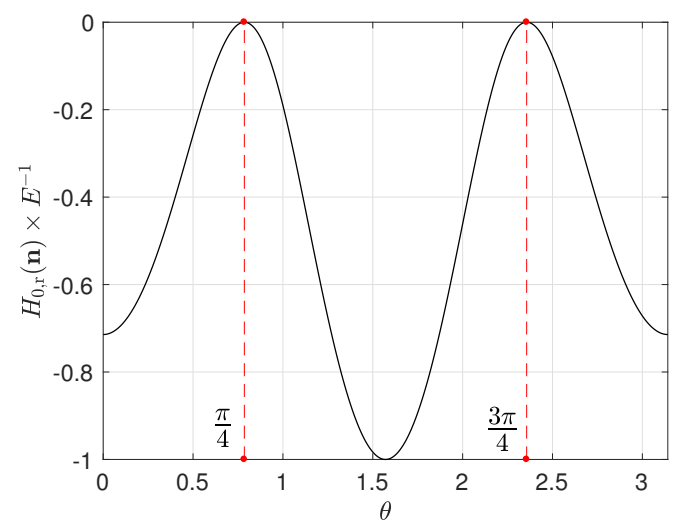

Figure 4: Graph of the critical hardening modulus (normalized by Young's modulus) on the right corner of the Tresca yield surface computed with normals of the form $\mathbf{n}=(\cos (\theta), \sin (\theta), 0)$ or $\mathbf{n}=(\cos (\theta), 0, \sin (\theta))$. The same curve was obtained on the left corner of the yield surface.

can be fulfilled for perfectly plastic or softening behaviors, i.e., for any $H_{0, r} \leq 0$. From Eqs. (95)-(97), it can be deduced that the determinant of the acoustic tensor vanishes for a perfectly plastic material and becomes negative for a softening behavior whenever the stress state lies on the right corner of the yield surface. Recall that the critical hardening modulus is defined such that it yields a singular acoustic tensor (see section 3), it is found that on the right corner the yield surface:

$$
H_{0, r}^{c r i t} \in[0,+\infty), \quad \mathbf{n}_{0, r}^{c r i t}=\left\{\begin{array}{llc} 
\pm \frac{1}{\sqrt{2}}\left(\mathbf{m}_{2} \pm \mathbf{m}_{3}\right), & \text { if } \quad H_{0, r} \geq 0 \\
\pm \frac{1}{\sqrt{2}}\left(\mathbf{m}_{1} \pm \mathbf{m}_{2}\right), & \text { if } \quad H_{0, r}=0 \\
\pm \frac{1}{\sqrt{2}}\left(\mathbf{m}_{1} \pm \mathbf{m}_{3}\right), & \text { if } \quad H_{0, r}=0
\end{array}\right.
$$

where it should be emphasized that the eigenvectors $\mathbf{m}_{2}$ and $\mathbf{m}_{3}$ are arbitrary unit vectors orthogonal to $\mathbf{m}_{1}$.

On the left corner of the yield surface, $(\boldsymbol{\sigma}, R) \in \partial \mathbb{E}_{l}$, the set of active mechanisms is given by $\mathbb{J}=\{1,2\}$. The eigenvalues of $\mathbf{N}^{1}$ and $\mathbf{N}^{2}$ are given by $N_{1}^{1}=a_{1}, N_{2}^{1}=a_{2}, N_{3}^{1}=a_{3}$, and $N_{1}^{2}=a_{2}, N_{2}^{2}=a_{1}, N_{3}^{2}=a_{3}$. The entries of the matrix $\left[\pi_{l}^{(0)}(\mathbf{n})\right]$ take the form

$$
\left[\pi_{l}^{(0)}(\mathbf{n})\right]=4 \mu\left[\begin{array}{cc}
\alpha\left(n_{1}^{2}-n_{3}^{2}\right)^{2}+n_{1}^{2}+n_{3}^{2} & \alpha\left(n_{1}^{2}-n_{3}^{2}\right)\left(n_{2}^{2}-n_{3}^{2}\right)+n_{3}^{2} \\
\alpha\left(n_{1}^{2}-n_{3}^{2}\right)\left(n_{2}^{2}-n_{3}^{2}\right)+n_{3}^{2} & \alpha\left(n_{2}^{2}-n_{3}^{2}\right)^{2}+n_{2}^{2}+n_{3}^{2}
\end{array}\right] .
$$



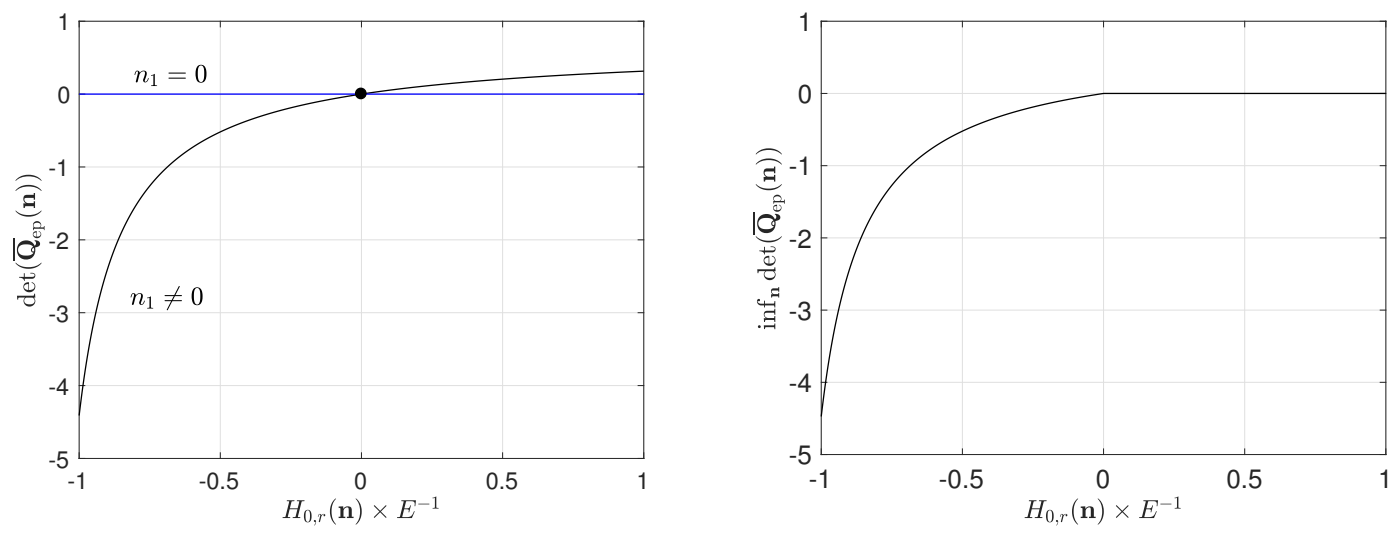

Figure 5: Left: Graphs of the determinant of the normalized acoustic tensor $\overline{\mathbf{Q}}_{e p}=\mathbf{Q}_{e l}^{-1} \mathbf{Q}_{e p}$ on the right corner of the Tresca yield surface with respect to the normalized hardening modulus $H_{0, r}(\mathbf{n}) \times E^{-1}$. The normal vectors are chosen as: $n_{1} \neq 0$, i.e., $\mathbf{n}=(\cos (\theta), \sin (\theta), 0)$ or $\mathbf{n}=(\cos (\theta), 0, \sin (\theta))$, and: $n_{1}=0$, i.e., $\mathbf{n}=(0, \cos (\theta), \sin (\theta))$. Similar trends were obtained on the left corner of the yield surface. Right: determinant of the normalized acoustic tensor obtained with the algorithm described in section 3.3 .

Similar results are obtained for the critical hardening modulus as in the case of the right corner. The matrix $\left[\pi_{l}^{(0)}(\mathbf{n})\right]-[\chi]$ is singular whenever $n_{3}^{2}=n_{2}^{2}$ and $n_{1}=0, n_{3}^{2}=n_{1}^{2}$ and $n_{2}=0$, or $n_{3}=0$ and $n_{1}^{2}=n_{2}^{2}$. Similarly to Eq. 950, the conditions

$$
\left.\operatorname{det}\left(\mathbf{Q}_{e p}\left(\mathbf{n}_{0, l}\right)\right)=0 \quad \text { and } \quad \operatorname{det}\left(\left[\pi^{(0)}\left(\mathbf{n}_{0, l}\right)\right]-[\chi]-H_{l}\left(\mathbf{n}_{0, l}\right)\left[i_{2}\right]\right)\right)=0
$$

are met for

$$
H_{0, l} \in \mathbb{R}, \quad \mathbf{n}_{0, l}=\left(n_{1}, n_{2}, 0\right), \quad n_{1}^{2}=n_{2}^{2}, \quad n_{1}^{2}+n_{2}^{2}=1
$$

The acoustic tensor can also be negative semi-definite for perfectly plastic or softening behaviors. Furthermore, the conditions given by Eq. (101) can be satisfied for the hardening modulus $H_{0, l}=0$ and normal vectors of the form

$$
\mathbf{n}_{0, l}=\frac{1}{\sqrt{2}}\left[\begin{array}{c}
0 \\
\pm 1 \\
\pm 1
\end{array}\right]_{\left(\mathbf{m}_{i}\right)_{i=1}^{3}} \quad \text { or } \quad \mathbf{n}_{0, l}=\frac{1}{\sqrt{2}}\left[\begin{array}{c} 
\pm 1 \\
0 \\
\pm 1
\end{array}\right]_{\left(\mathbf{m}_{i}\right)_{i=1}^{3}} .
$$

Please note that in this case the principal directions $\mathbf{m}_{1}$ and $\mathbf{m}_{2}$ can be any vectors in $\mathbb{R}^{3}$ that are orthogonal to $\mathbf{m}_{3}$. Hence, the critical hardening modulus $H_{0, l}^{\text {crit }}$ and the normal 

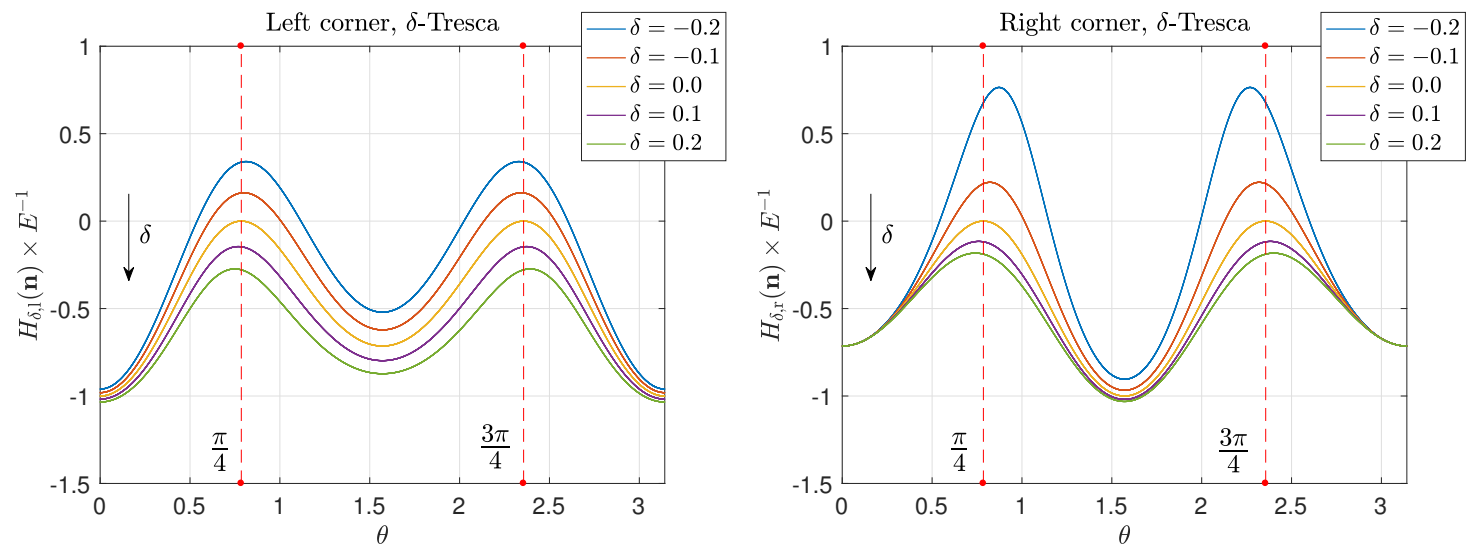

Figure 6: Graphs of the hardening modulus on the left and right corner of the $\delta$-Tresca model. The normal $\mathbf{n}$ is parameterized as follows: $\mathbf{n}=(\cos (\theta), \sin (\theta), 0)$ on the right corner, and $\mathbf{n}=(0, \cos (\theta), \sin (\theta))$ on the left corner.

vector $\mathbf{n}_{0, l}^{\text {crit }}$ that yield a singular acoustic tensor are given by

$$
H_{0, l}^{c r i t} \in[0,+\infty), \quad \mathbf{n}_{0, l}^{c r i t}=\left\{\begin{array}{ccc} 
\pm \frac{1}{\sqrt{2}}\left(\mathbf{m}_{1} \pm \mathbf{m}_{2}\right), & \text { if } \quad H_{0, l} \geq 0 \\
\pm \frac{1}{\sqrt{2}}\left(\mathbf{m}_{2} \pm \mathbf{m}_{3}\right), & \text { if } \quad H_{0, l}=0 \\
\pm \frac{1}{\sqrt{2}}\left(\mathbf{m}_{1} \pm \mathbf{m}_{3}\right), & \text { if } \quad H_{0, l}=0 .
\end{array}\right.
$$

The standard Tresca yield criterion leads to loss of ellipticity whenever the stress lies on the smooth portion of the surface if the hardening modulus vanishes (see Eq. (88)). In addition, the acoustic tensor is singular on both corners of the yield surface and becomes negative definite for perfectly plastic or softening behaviors.

\subsubsection{Critical hardening modulus on the corners of the $\delta$-Tresca and $\tau$-Tresca yield surfaces}

The same analysis can be carried out for the $\delta$-Tresca and $\tau$-Tresca models. The entries of the matrices $\left[\pi_{r}^{(\delta)}(\mathbf{n})\right],\left[\pi_{l}^{(\delta)}(\mathbf{n})\right],\left[\pi_{r}^{(\tau)}(\mathbf{n})\right]$, and $\left[\pi_{l}^{(\tau)}(\mathbf{n})\right]$ can be obtained using Eqs. 42 and $(79)$ but their expressions are reported in Appendix Appendix B for the sake of clarity. Graphs of the hardening modulus are shown in Figs.6 and Fig.7 for a normal vector $\mathbf{n}$ of the forms $\mathbf{n}=(\cos (\theta), \sin (\theta), 0)$ and $\mathbf{n}=(0, \cos (\theta), \sin (\theta))$. For $\delta=0$ and $\tau=0$, it can be seen that the results for the standard Tresca model are recovered. Non-zeros values of $\delta$ and $\tau$ allow for modifying the maximum value reached by the hardening modulus with 

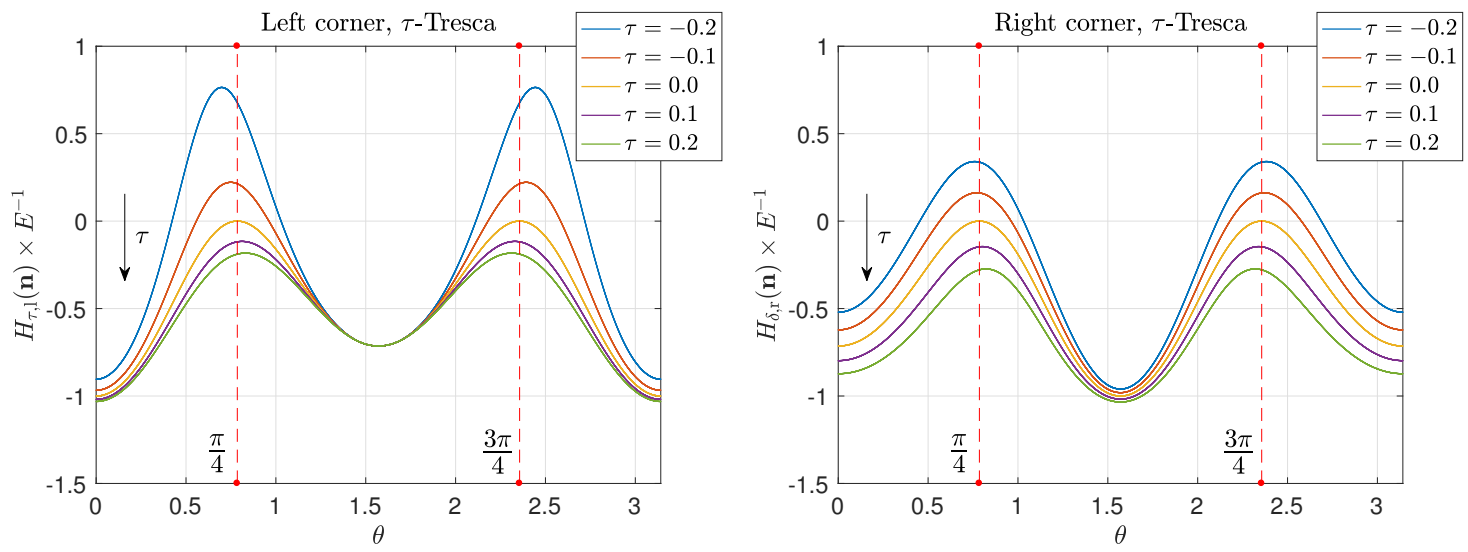

Figure 7: Graphs of the hardening modulus on the left and right corner of the $\tau$-Tresca model. The normal $\mathbf{n}$ is parameterized as follows: $\mathbf{n}=(\cos (\theta), \sin (\theta), 0)$ on the right corner, and $\mathbf{n}=(0, \cos (\theta), \sin (\theta))$ on the left corner.

respect to the orientation of the shear band. In particular, for negative values of $\delta$ or $\tau$, the maximum value significantly increases.

However, these observations are conditioned by the choice of the normal vector $\mathbf{n}$. It was found that two families of normal vectors $\mathbf{n}$ are relevant by performing numerical investigations based on the procedure presented in section 3.3. Depending on the values of $\delta$ or $\tau$, the acoustic tensor can be singular or negative semi-definite for any real values of the hardening modulus. This observation is illustrated in Figs. 8 and 9 where the graphs of loss of ellipticity indicators with respect to the hardening modulus are shown on the right corners of the yield functions.

- In the left panels of Figs. 8 and 9 , the determinant of the normalized acoustic tensor $\overline{\mathbf{Q}}_{e p}$ is computed with respect to the hardening modulus and for two chosen families of normal vectors.

- In the right panels of Figs. 8 and 9, $\inf _{\mathbf{n} \in \mathcal{S}} \operatorname{det}\left(\mathbf{Q}_{e p}(\mathbf{n})\right)$ is computed with respect to the hardening modulus using the strategy described in section 3.3 .

It can be deduced that on the right corner of the yield surface, the $\delta$-Tresca model is highly unstable for any $\delta \in]-1,0[$ while loss of ellipticity can be postponed for any $\delta \in] 0,1 / 2[$. 
In the case of the $\tau$-Tresca model, it can be seen in Fig. 9 that the effect of the parameter $\tau$ is similar but reversed. Ellipticity is not achieved for all $\tau \in] 0,1 / 2$ [ while negative values of $\tau \in]-1,0[$ allow for increasing the critical hardening modulus, i.e., prepone loss of ellipticity. Similar results are obtained on the left corners of the yield functions, as shown in Fig. 10. As a conclusion to this section, the presence of the corners on the yield surface together with the parameters $\delta$ and $\tau$ allow for postponing loss of ellipticity (i.e., decreasing the critical hardening modulus), or advancing loss of ellipticity (i.e., increasing the critical hardening modulus). These results are summarized in Tab.1 and graphs of the critical hardening moduli with respect to $\delta$ and $\tau$ are depicted in Fig.11. Several situations can be encountered, namely:

- For $\delta \in]-1,0[$, loss of ellipticity is advanced on the left corner and the associated critical hardening modulus is positive. However, loss of ellipticity is postponed on the smooth portion (see Eq. (89)), and the acoustic tensor is negative definite on the right corner.

- For $\delta \in] 0,1 / 2[$, loss of ellipticity is postponed on right corner and the corresponding critical hardening modulus is negative. Loss of ellipticity is also postponed on the smooth portion but the acoustic tensor is negative definite on the left corner.

- Similarly, $\tau \in]-1,0[$ allows for advancing loss of ellipticity on the right corner with a positive critical hardening modulus. Loss of ellipticity is postponed on the smooth portion but the acoustic tensor is negative definite on the left corner.

- Finally, for $\tau \in] 0,1 / 2[$, loss of ellipticity is postponed on the left corner and on the smooth portion. The acoustic tensor is negative definite on the right corner. 

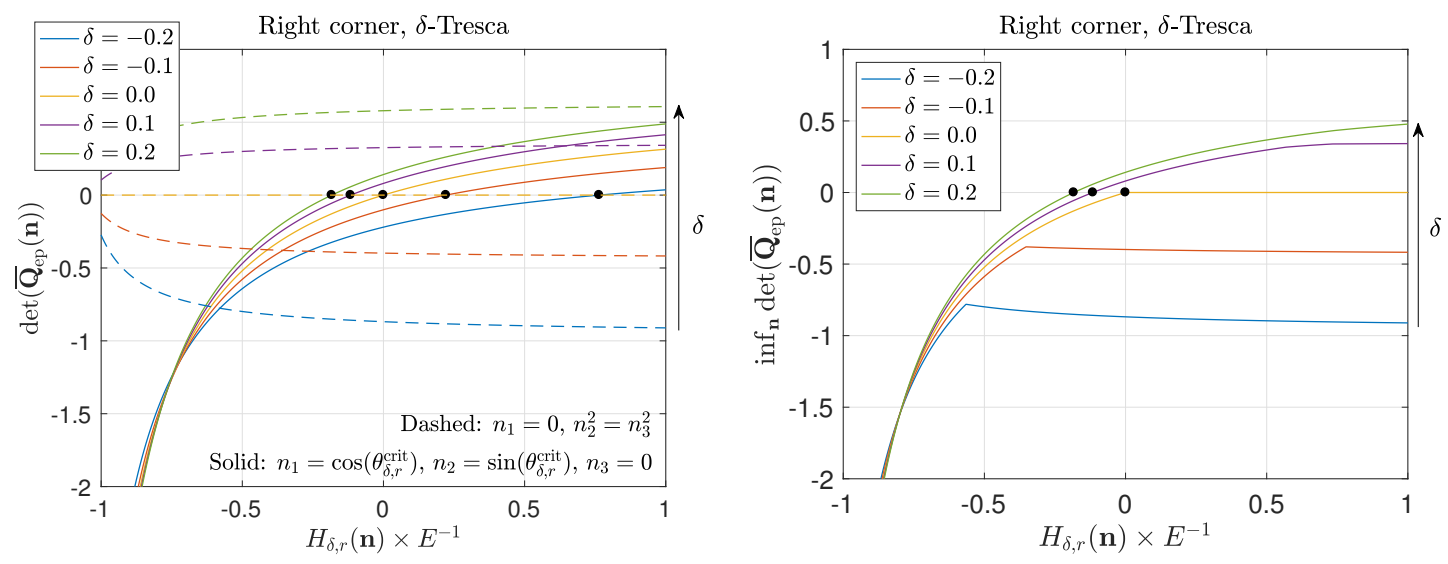

Figure 8: Graphs of the determinant of the normalized acoustic tensor $\overline{\mathbf{Q}}_{e p}=\mathbf{Q}_{e l}^{-1} \mathbf{Q}_{e p}$ with respect to the hardening modulus on the right corner of the $\delta$-Tresca model for several values of $\delta$. Left: the determinant of the normalized acoustic tensor is computed using two parameterization of the normal $\mathbf{n}$ : $\mathbf{n}=\left(\cos \left(\theta_{\delta, r}^{\text {crit }}\right), \sin \left(\theta_{\delta, r}^{\text {crit }}\right), 0\right)$ with $\theta_{\delta, r}^{\text {crit }}$ being the angle maximizing the hardening modulus in Fig. 6 , and $\mathbf{n}$ such that $n_{1}=0, n_{2}^{2}=n_{3}^{2}=1 / 2$. Right: determinant of the normalized acoustic tensor obtained with the algorithm described in section 3.3 .
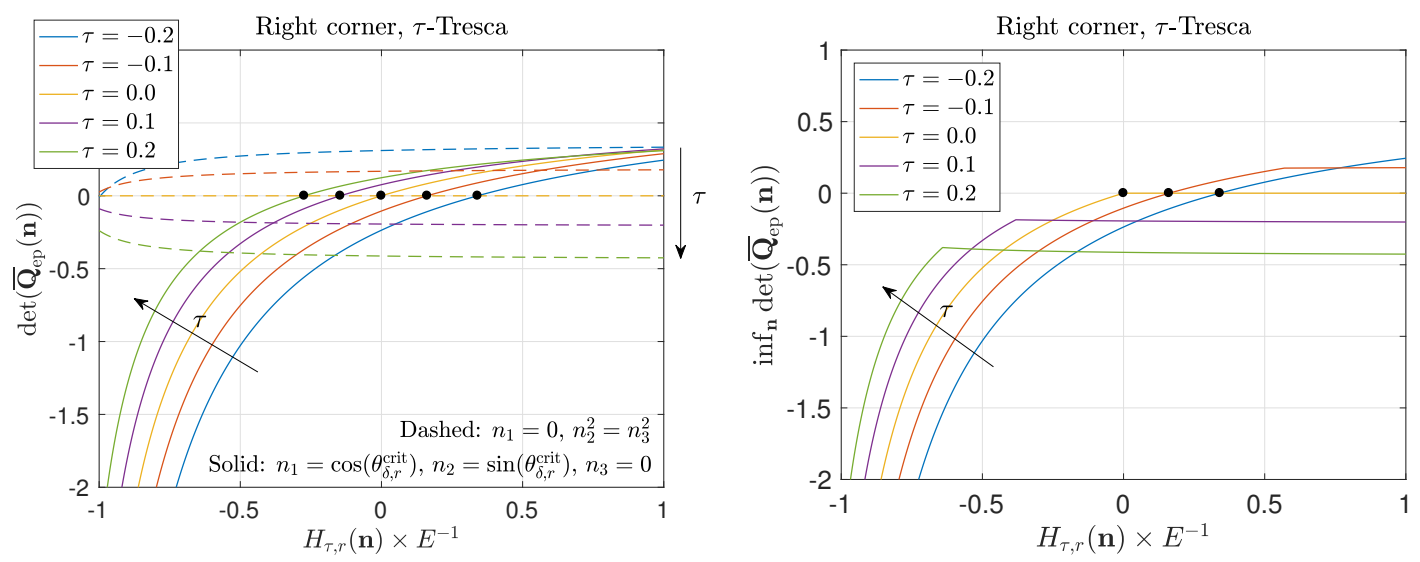

Figure 9: Graphs of the determinant of the normalized acoustic tensor $\overline{\mathbf{Q}}_{e p}=\mathbf{Q}_{e l}^{-1} \mathbf{Q}_{e p}$ on the right corner of the $\delta$-Tresca model for several values of $\delta$. Two parameterization of the normal $\mathbf{n}_{\delta, r}^{\text {crit }}$ are considered: $\mathbf{n}=\left(\cos \left(\theta_{\delta, r}^{\text {crit }}\right), \sin \left(\theta_{\delta, r}^{\text {crit }}\right), 0\right)$ with $\theta_{\delta, r}^{\text {crit }}$ being the angle maximizing the hardening modulus in Fig. 7 , and $\mathbf{n}=\left(n_{1}, n_{2}, n_{3}\right)_{\left(\mathbf{m}_{i}\right)_{i=1}^{3}}$ such that $n_{1}=0, n_{2}^{2}=n_{3}^{2}=1 / 2$. 

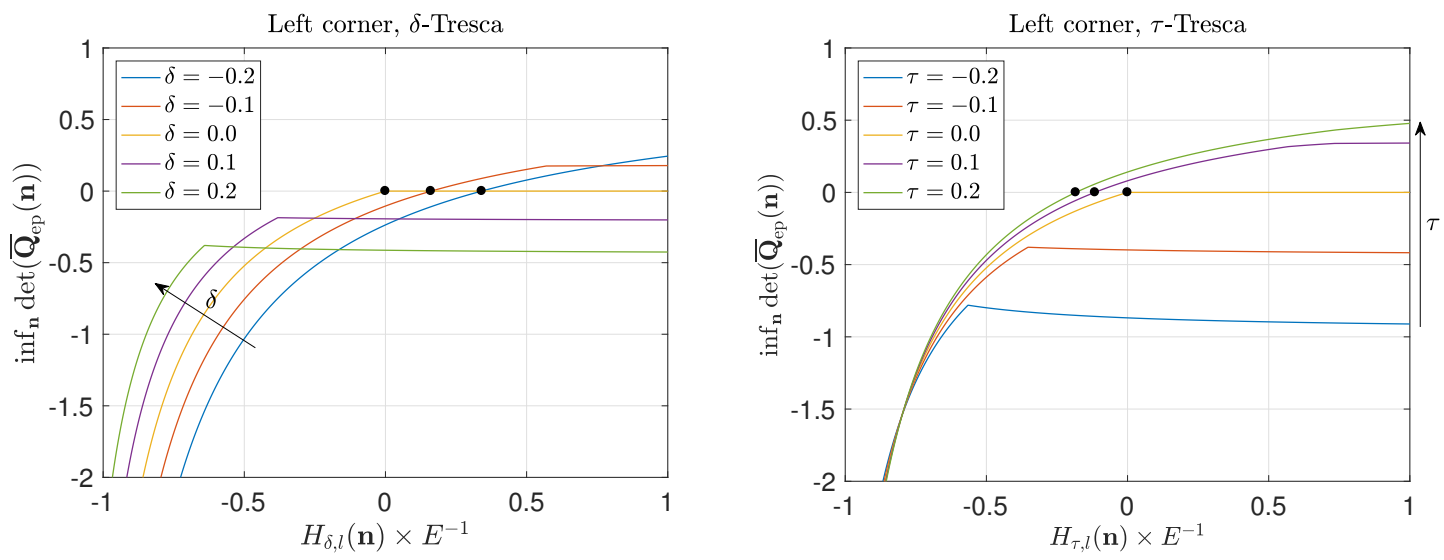

Figure 10: Graphs of the determinant of the normalized acoustic tensor $\overline{\mathbf{Q}}_{e p}=\mathbf{Q}_{e l}^{-1} \mathbf{Q}_{e p}$ with respect to the hardening modulus on the left corners of the $\delta$-Tresca and $\tau$-Tresca models.
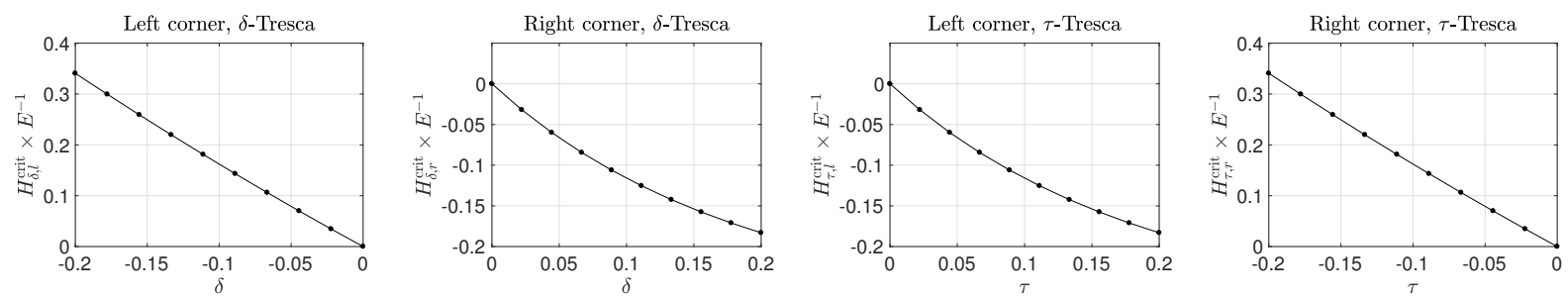

Figure 11: Graphs of the critical hardening moduli with respect to $\delta$ and $\tau$.

Table 1: Summary of the critical hardening moduli. Whenever the condition $\operatorname{det}\left(\mathbf{Q}_{e p}(\mathbf{n})\right)=0$ is not reached for given values of $\delta$ and $\tau$, the critical hardening modulus does not exist ( $\nexists)$.

\begin{tabular}{llll}
\hline model & $\boldsymbol{\sigma} \in \partial \mathbb{E}_{s}$ & $\boldsymbol{\sigma} \in \partial \mathbb{E}_{l}$ & $\boldsymbol{\sigma} \in \partial \mathbb{E}_{r}$ \\
\hline Tresca & $H_{0, s}^{\text {crit }}=0$ & $H_{0, l}^{\text {crit }} \in[0, \infty)$ & $H_{0, r}^{\text {crit }} \in[0, \infty)$ \\
\hline$\delta$-Tresca & $H_{\delta, s}^{c r i t}=-E \delta^{2}$ & $\left.H_{\delta, l}^{c r i t}=\nexists, \delta \in\right] 0,1 / 2[$ & $\left.H_{\delta, r}^{\text {crit }}<0, \delta \in\right] 0,1 / 2[$ \\
$\delta \in]-1,1 / 2[$ & $\left.H_{\delta, l}^{\text {crit }}>0, \delta \in\right]-1,0[$ & $\left.H_{\delta, r}^{\text {crit }}=\nexists, \delta \in\right]-1,0[$ \\
\hline$\tau$-Tresca & $H_{\tau, s}^{\text {crit }}=-E \tau^{2}$ & $\left.H_{\tau, l}^{\text {crit }}<0, \tau \in\right] 0,1 / 2[$ & $\left.H_{\tau, r}^{\text {crit }}=\nexists, \tau \in\right] 0,1 / 2[$ \\
$\tau \in]-1,1 / 2[$ & & $\left.H_{\tau, l}^{\text {crit }}=\nexists, \tau \in\right]-1,0[$ & $\left.H_{\tau, r}^{\text {crit }}>0, \tau \in\right]-1,0[$ \\
\hline
\end{tabular}




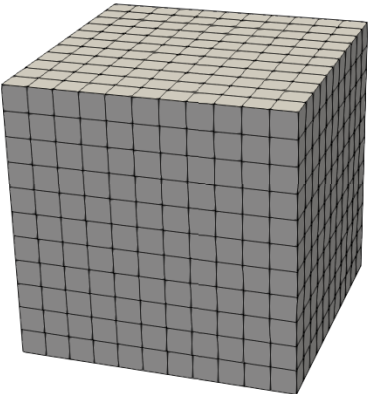

(a) Cube

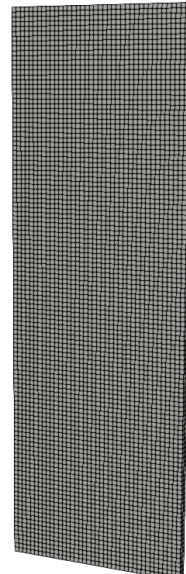

(b) Thin plate

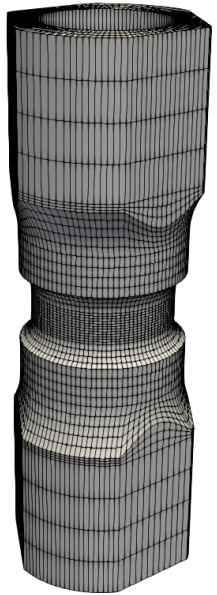

(c) Experimental tubular specimen

Figure 12: Finite element meshes of the cube (using 10 elements in each direction) and thin plate (using 80, 240, and 4 quadratic elements along the width, height, and thickness, respectively), and geometry of the tubular specimen taken from [48] and 9 .

\section{Numerical examples}

In the next section, finite element simulations are performed in order to illustrate the proposed analysis. For simplicity, only the $\delta$-Tresca model will be considered. Three different geometries are introduced, namely, a cube in section 5.2, and a thin plate that measures $L=12 \mathrm{~mm}$ in height, $L / 3 \mathrm{~mm}$ in width, and is $L / 6 \mathrm{~mm}$ thick in section 5.3 . Finally, an experimental tubular specimen is considered in section 5.4. The geometries are discretized with 20-noded hexahedral elements and a reduced integration scheme is used within each element to reduce locking phenomena. Finite element meshes of the geometries are shown in Fig.12. Finite element simulations are performed with the non-linear material and structure analysis suite Zset (http://zset-software.com/). A nonlinear hardening function $\hat{p} \mapsto$ $R(\hat{p})$ is chosen as

$$
R(\hat{p})=R_{0}+S \hat{p}+Q(1-\exp (-b \hat{p}))
$$


for which the hardening modulus $\hat{p} \mapsto H(\hat{p}) \equiv d R(\hat{p}) / d \hat{p}$ takes the form

$$
H(\hat{p})=S+Q b \exp (-b \hat{p})
$$

In the above equations, $R_{0}$ is a constant yield stress, $Q$, and $b$ are two hardening parameters chosen as

$$
R_{0}=1000 \mathrm{MPa}, \quad Q=100 \mathrm{MPa}, \quad b=300
$$

and the linear hardening modulus $S$ is given by

$$
S=-1000 \mathrm{MPa} .
$$

Young's modulus and Poisson's ratio are set to $E=210,000 \mathrm{MPa}$ and $\nu=0.3$. Imperfections are inserted by imposing a reduced yield stress $R_{0}^{\prime}=999.0 \mathrm{MPa}$ in chosen elements of the cube and thin plate. The objective of the imperfection is to fix the location of the strain localization band which will form and avoid complex interactions with the boundaries. This section is organized as follows. First, in section 5.1, the implicit integration scheme used for the time integration of the elasto-plastic constitutive problem is described. Then, in the remaining sections, localization and loss of ellipticity are studied for various plastic thresholds and the considered three geometries.

\subsection{Implicit integration scheme}

The elasto-plastic constitutive problem is solved with an implicit integration scheme. A general implicit integration scheme for multisurface plasticity has been proposed by [27] and recently reformulated in principal stress space by [30]. It should be noted that there also exist fully implicit integration schemes for the Tresca criterion with isotropic hardening [28, 36] and for the yield function given by Eq. (68) [47]. Herein, we have recourse to the general procedure described by [27] in order to easily accommodate any yield function expressed in terms of principal stresses. At a fixed Gauss point, given $\varepsilon_{n}^{e}, p_{n}$, and $\Delta \varepsilon_{n+1}$ at a given time increment $t_{n}$, we seek the updated tensor $\varepsilon_{n+1}^{e}$ and the increments of the Lagrange multipliers $\Delta \gamma_{j, n+1}$ for $j=1, \ldots, m$. A sketch of the algorithm is given in the box Algorithm 1. It consists in solving the residual equation $\mathbf{r} \equiv\left(\mathbf{r}^{e}, \mathbf{r}^{\phi}\right)=\mathbf{0}$ for the principal 
stresses $\hat{\boldsymbol{\sigma}}_{n+1}$, the internal variable $p_{n+1}$, and the set of Lagrange multipliers $\left\{\Delta \gamma_{j, n+1}\right\}_{j \in \mathbb{J}}$ where

$$
\mathbf{r}^{e}=[C]^{-1}\left(\hat{\boldsymbol{\sigma}}_{n+1}-\hat{\boldsymbol{\sigma}}_{n+1}^{\text {trial }}\right)+\sum_{j \in \mathbb{J}} \Delta \gamma_{j, n+1} \hat{\mathbf{n}}_{j}, \quad r_{j}^{\phi}=\phi_{j}\left(\hat{\boldsymbol{\sigma}}_{n+1}, R_{n+1}\right), \quad j \in \mathbb{J},
$$

where

- the vectors gathering the ordered principal stresses and elastic strains are related by $\hat{\boldsymbol{\sigma}}_{n+1}=[C] \hat{\boldsymbol{\varepsilon}}_{n+1}$, in which the matrix $[C]$ can easily be deduced from the entries of the fourth-order elasticity tensor $\mathbb{C}$;

- the updated yield stress is given by $R_{n+1}=R\left(p_{n}+\sum_{j \in \mathbb{J}} \Delta \gamma_{j, n+1}\right)$;

- the yield function has been written in terms of the principal stresses, i.e.,

$$
\hat{\phi}_{j}(\hat{\boldsymbol{\sigma}}, r)=\phi_{j}\left(\sum_{i=1}^{3} \hat{\sigma}_{i} \mathbf{M}_{i}, r\right), \quad j=1, \ldots, m,
$$

for any yield stress $r$, where $\mathbf{M}_{i}, i=1,2,3$, denotes the elements of the eigenbasis of the stress tensor,

- and the vectors $\hat{\mathbf{n}}_{1}, \hat{\mathbf{n}}_{2}$ and $\hat{\mathbf{n}}_{3}$ correspond to the first-order partial derivatives of the yield function with respect to the ordered principal stresses, i.e.,

$$
\hat{\mathbf{n}}_{1}=\left[\begin{array}{c}
a_{1} \\
a_{2} \\
a_{3}
\end{array}\right]_{\left(\mathbf{e}_{i}\right)_{i=1}^{3}}, \quad \hat{\mathbf{n}}_{2}=\left[\begin{array}{l}
a_{2} \\
a_{1} \\
a_{3}
\end{array}\right]_{\left(\mathbf{e}_{i}\right)_{i=1}^{3}}, \quad \hat{\mathbf{n}}_{3}=\left[\begin{array}{l}
a_{1} \\
a_{3} \\
a_{2}
\end{array}\right]_{\left(\mathbf{e}_{i}\right)_{i=1}^{3}} .
$$

By having recourse to a Newton-Raphson procedure, one has to solve the following sequence until convergence has been met:

$$
k \geq 0: \quad\left[J\left(\boldsymbol{\eta}^{(k)}\right)\right]\left(\boldsymbol{\eta}^{(k+1)}-\boldsymbol{\eta}^{(k)}\right)=-\mathbf{r}\left(\boldsymbol{\eta}^{(k)}\right), \quad \boldsymbol{\eta}^{(k)} \equiv\left(\hat{\boldsymbol{\sigma}}_{n+1}^{(k)}, \boldsymbol{\alpha}_{n+1}^{(k)},\left(\Delta \gamma_{j, n+1}^{(k)}\right)_{j \in \mathbb{J}}\right)
$$

where $\left[J\left(\boldsymbol{\eta}^{(k)}\right)\right]$ denotes the jacobian matrix for a given value $\boldsymbol{\eta}^{(k)}$ of the unknowns. The jacobian matrix is populated by the partial derivatives of the residuals $\mathbf{r}^{e}$ and $\mathbf{r}^{\phi}$ with 
respect to $\hat{\boldsymbol{\sigma}}_{n+1}^{(k)}, p_{n+1}^{(k)}$, and $\left(\Delta \gamma_{j, n+1}^{(k)}\right)_{j \in \mathbb{J}}$. In addition, the consistent tangent modulus that is necessary for the global Newton-Raphson scheme (at the structural level) is computed using the systematic approach presented by, e.g., [49]. Once the Newton-Raphson procedure has converged for some $k^{\star}$, the Jacobian matrix $\left[J\left(\boldsymbol{\eta}^{\left(k^{\star}\right)}\right)\right]$ is assembled for the solution $\boldsymbol{\eta}^{\left(k^{\star}\right)}$. The consistent tangent matrix is then given by the first $(6 \times 6)$ block of the inverse of the jacobian matrix $\left[J\left(\boldsymbol{\eta}^{\left(k^{\star}\right)}\right)\right]^{-1}$.

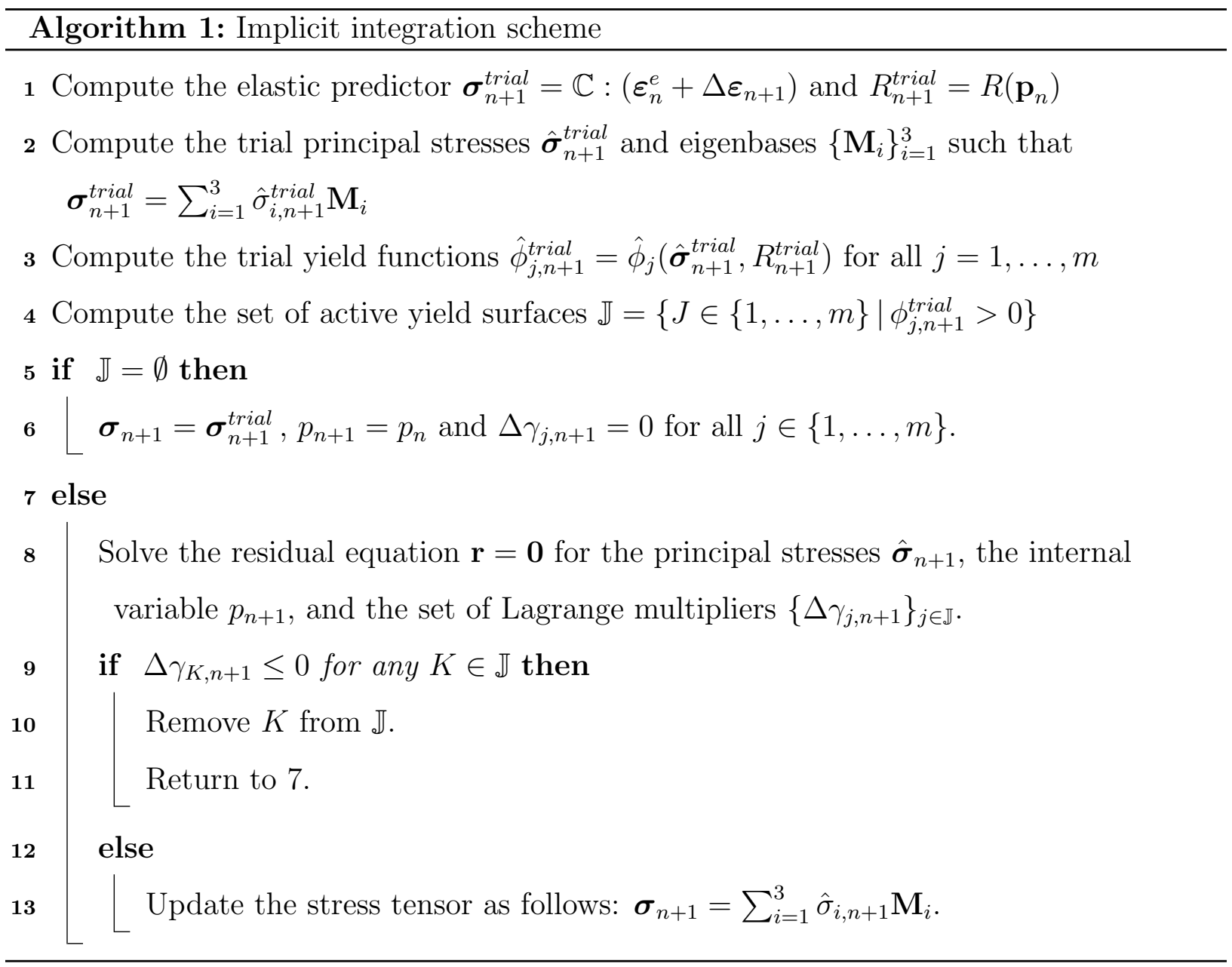

\subsection{Softening cube undergoing tension}

We start by comparing the results obtained with a cube using the Tresca and von Mises yield functions. A vertical displacement $u_{d}$ is applied to the top edge and boundary conditions at the bottom of the cube are such that tensile homogeneous deformations take place 
in the elastic regime. In this setting, we expect the stress state to lie on the right corner of the yield surface at most Gauss points of the finite element mesh. The parameter $S$ in Eq. (105) is chosen as $S=-1000 \mathrm{MPa}$ in order to enforce a slightly softening behavior.

Figure 13 shows the reaction forces on the top surface of the cube for six different plastic thresholds, namely, for the von Mises, Tresca, and $\delta$-Tresca model with four different values of the parameter $\delta$. In addition, in Fig. 13, the prescribed deformation $\varepsilon_{E}$ for which loss of ellipticity occurs for the first time in the finite element mesh is shown. The first occurrence of lost of ellipticity is obtained as follows. At each time step, the minimum of the loss of ellipticity indicator over all the Gauss points is computed. This minimum value is considered to be negative as soon as it gets lower than or equal to $-10^{-14}$. The prescribed deformation $\varepsilon_{D}$ for which the resultant force reaches its maximum value is also given. In the case of the von Mises model, it is seen that loss of ellipticity occurs well after the resultant force has reached its maximum value. In contrast, in the case of the $\delta$-Tresca models with $\delta \in\{-0.2,-0.1,0.0,0.1,0.2\}$, it is observed that loss of ellipticity occurs slightly before the maximum resultant force. In Fig. 14, the graph of the minimum value of the loss of ellipticity indicator is shown with respect to the prescribed deformation $u_{d} / L$. The minimum is taken over all the Gauss points of the finite element mesh. A value equal to 1 means that ellipticity has not decreased. It can be seen that the indicators suddenly decrease as soon as the cube plastifies. In the case of the von Mises model, the indicator drops to a positive value then smoothly decreases. In contrast, in the case of the Tresca model, the indicator directly drops to zero then smoothly decreases towards negative values. This numerical observation can be explained by means of Eq. (99). Indeed, on the right corner of the Tresca yield function, we expect the acoustic tensor to be singular as soon as the material plastifies.

Figure 15 shows that strain remains almost homogeneous using the von Mises criterion, whereas strain localization bands tend to form according to Tresca and $\delta$-Tresca criteria. The same fields are shown in Fig. 16 in the case of the Tresca model. In contrast to the von Mises model, a distinctive strain localization band forms on the top surface of the cube. The indicator of loss of ellipticity decreases where localization occurs.

It is well-known that loss of ellipticity for von Mises plasticity under tension can only 


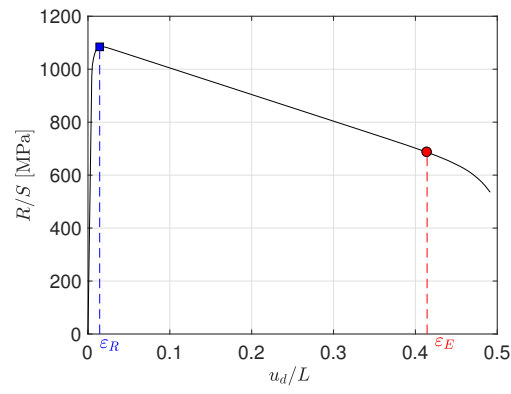

(a) von Mises

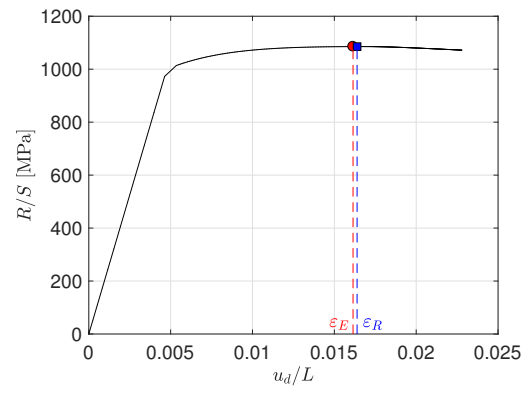

(b) Tresca

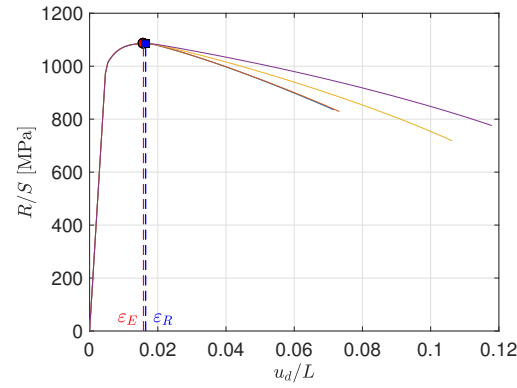

(c) $\delta$-Tresca

Figure 13: Cube undergoing simple tension: graphs of the force resultant $R$ with respect to the prescribed deformation $u_{d} / L$ with $L=4 \mathrm{~mm}$. The deformations $\varepsilon_{E}$ and $\varepsilon_{R}$ correspond to: the first occurrence of loss of ellipticity and to when $R$ reaches its maximum value.

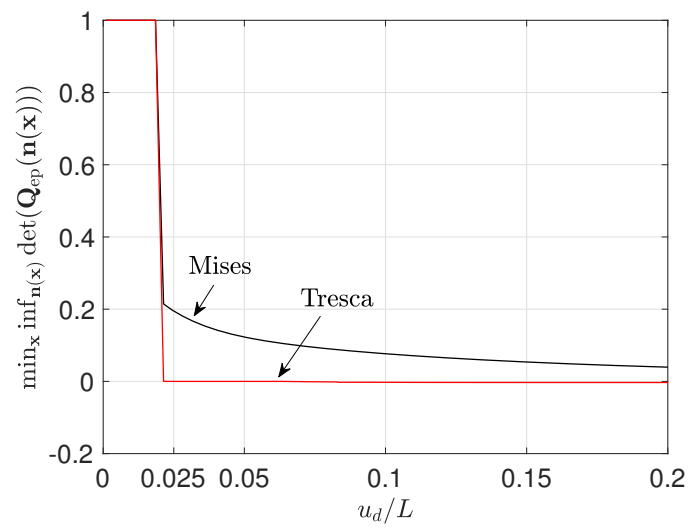

Figure 14: Cube undergoing simple tension: graph of minimum value of the loss of ellipticity indicator over the Gauss points of the finite element meshes, with respect to the prescribed deformation $u_{d} / L$ with $L=4 \mathrm{~mm}$. 


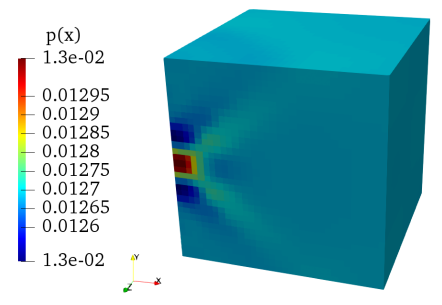

(a) $u_{d} / L=\varepsilon_{R}$

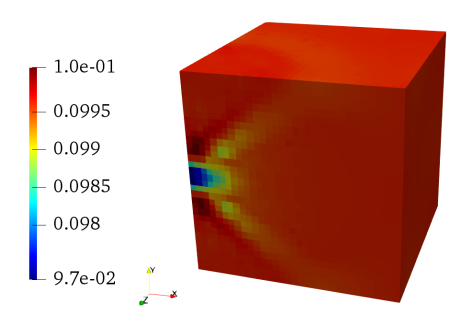

(d) $u_{d} / L=\varepsilon_{R}$

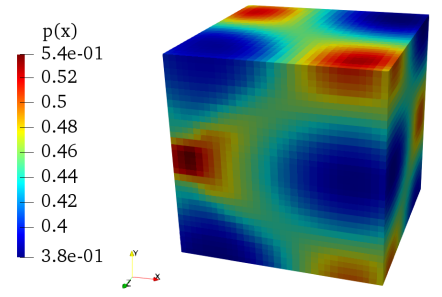

(b) $u_{d} / L=\varepsilon_{E}$

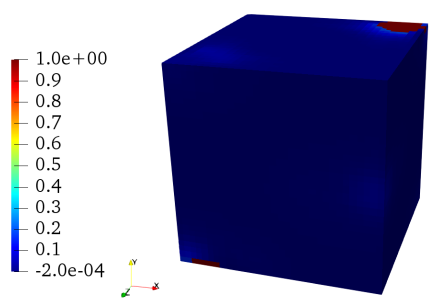

(e) $u_{d} / L=\varepsilon_{E}$

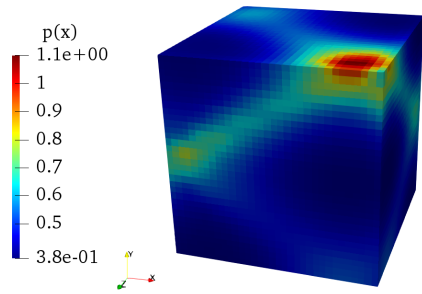

(c) $u_{d} / L=0.49$

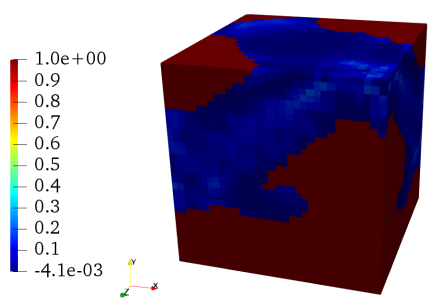

(f) $u_{d} / L=0.49$

Figure 15: Cumulative plastic strain $\mathbf{x} \mapsto p(\mathbf{x})$ (first row) and loss of ellipticity indicator $\mathbf{x} \mapsto$ $\inf _{\mathbf{n}(\mathbf{x}) \in \mathcal{S}} \operatorname{det}\left(\mathbf{Q}_{e p}(\mathbf{n}(\mathbf{x}))\right)$ (second row) in the case of a von Mises model at three overall strain levels indicated in Fig. 13(a).

occur for a very low value of softening modulus, namely $-E / 4$, as discussed by, e.g., [50]. Here, the presence of an imperfection leads to a heterogeneous deformation field and loss of ellipticity occurs even in the case of the von Mises model. However, it occurs for a much larger prescribed displacement and much higher deformations that are unrealistic under the assumptions of small strains.

\subsection{Thin softening plate undergoing tension}

In this second illustration, we consider the case of a thin plate. The boundary conditions are the same as in the case of the cube addressed in the previous section, namely, there are such that homogeneous simple tension takes place in the absence of localization. The parameter $S$ in Eq. (105) is chosen as $S=-1000 \mathrm{MPa}$ as in the previous section. The resultant forces on the top surface of the plate are shown in Fig.17 for the von Mises and $\delta$-Tresca models. As in the case of the cube, loss of ellipticity occurs late after the resultant 


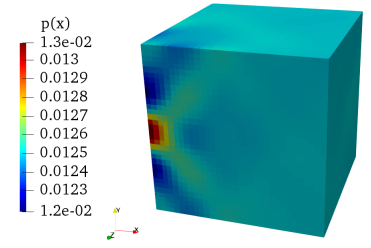

(a) $u_{d} / L=\varepsilon_{R}$

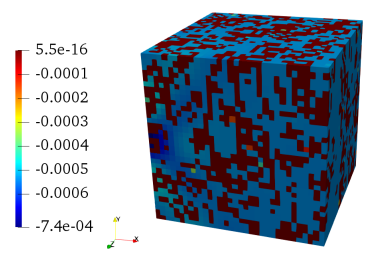

(e) $u_{d} / L=\varepsilon_{R}$

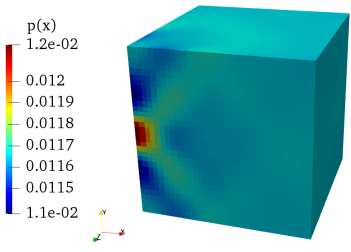

(b) $u_{d} / L=\varepsilon_{E}$

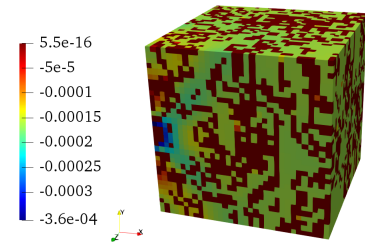

(f) $u_{d} / L=\varepsilon_{E}$

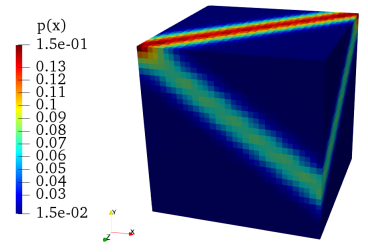

(c) $u_{d} / L=0.0325$

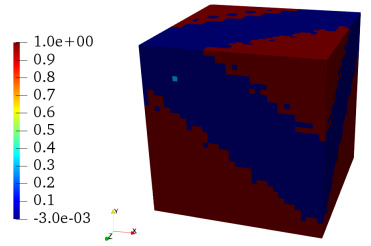

(g) $u_{d} / L=0.0325$
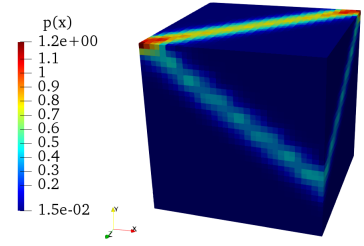

(d) $u_{d} / L=0.0825$

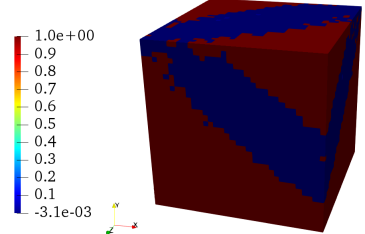

(h) $u_{d} / L=0.0825$

Figure 16: Cumulative plastic strain $\mathbf{x} \mapsto p(\mathbf{x})$ (first row) and loss of ellipticity indicator $\mathbf{x} \mapsto$ $\inf _{\mathbf{n}(\mathbf{x}) \in \mathcal{S}} \operatorname{det}\left(\mathbf{Q}_{e p}(\mathbf{n}(\mathbf{x}))\right)$ (second row) in the case of a Tresca model at four overall strain levels indicated in Fig. 13 (b).

force has reached its maximum value for the von Mises model. In contrast, for all the $\delta$-Tresca models, loss of ellipticity takes place before the maximum reaction force. In addition, it can be seen that loss of ellipticity is slightly postponed as $\delta$ increases. Views of the cumulative plastic strain field in the case of a Tresca model are shown in Fig.18. The localization band is found to be inclined across both the thickness and width. To explain this numerical result, we refer to Eq. (99) which shows that on the right corner of the Tresca yield surface, we may have

$$
\operatorname{det}\left(\mathbf{Q}_{e p}(\mathbf{n}(\mathbf{x}))\right)=0, \quad \mathbf{n}=\frac{1}{\sqrt{2}}\left[\begin{array}{c}
0 \\
\pm 1 \\
\pm 1
\end{array}\right]_{\left(\mathbf{m}_{i}\right)_{i=1}^{3}}, \quad \forall H(p(\mathbf{x})) \in[0,+\infty[
$$


or

$$
\operatorname{det}\left(\mathbf{Q}_{e p}(\mathbf{n}(\mathbf{x}))\right)=0, \quad \mathbf{n}(\mathbf{x})=\frac{1}{\sqrt{2}}\left[\begin{array}{c} 
\pm 1 \\
\pm 1 \\
0
\end{array}\right]_{\left(\mathbf{m}_{i}\right)_{i=1}^{3}} \quad \text { or } \quad \mathbf{n}(\mathbf{x})=\frac{1}{\sqrt{2}}\left[\begin{array}{c} 
\pm 1 \\
0 \\
\pm 1
\end{array}\right]_{\left(\mathbf{m}_{i}\right)_{i=1}^{3}} \quad, \quad \text { for } H(p(\mathbf{x}))=0
$$

at any point $\mathbf{x}$ in deformed configuration. If it is assumed that in this scenario of simple tension the eigenvalues $\left\{\mathbf{m}_{i}\right\}_{i=1}^{3}$ coincide with the canonical basis $\left(\mathbf{e}_{x}, \mathbf{e}_{y}, \mathbf{e}_{z}\right)$ of $\mathbb{R}^{3}$, then Eq. 110 means that a flat shear band across the plate's width may take place as well. Similarly, Eq. (111) states that an inclined shear band in the planes spanned by $\left(\mathbf{e}_{x}, \mathbf{e}_{y}\right)$ or $\left(\mathbf{e}_{x}, \mathbf{e}_{z}\right)$ may take place. However, it should be emphasized that the eigenvectors $\mathbf{m}_{2}$ and $\mathbf{m}_{3}$ can be arbitrary vectors than span the space orthogonal to the linear space $\left\{\alpha \mathbf{m}_{1}, \alpha \in \mathbb{R}\right\}$, hence leading to the observed shear band in Fig.18. As a comparison, in the case of a von Mises model, the shear band would also be inclined across the plate's width but flat across the thickness of the plate. In the latter case, necking takes place within the thickness of the band and no loss of ellipticity occurs.

To end this section, Fig. 19 shows the loss of ellipticity indicators in the case of various plastic thresholds. Similar results are obtained in most cases expect for the $\delta$-Tresca model with $\delta=-0.2$ and the von Mises model. For the latter, it is not surprising that ellipticity is not lost at $u_{d} / L \approx 0.0175$ since loss of ellipticity occurs for $u_{d} / L \geq \varepsilon_{E}$ with $\varepsilon_{E}>0.0175$ (see Fig.17). In the case of the $\delta$-Tresca model with $\delta=-0.2$, it is found that a flat shear band takes place across the plate's width. Recall that for negative values of $\delta$ and any real hardening modulus there exists a normal vector $\mathbf{n}$ such that the acoustic tensor $\mathbf{Q}_{e p}(\mathbf{n})$ is negative definite (see Fig. 8). We infer that for $\delta=-0.2$, we obtained a shear band orientation $\mathbf{n}$ with entries $n_{1}=0, n_{2}^{2}=n_{3}^{2}$ in the principal basis (that may coincide with the canonical basis). 


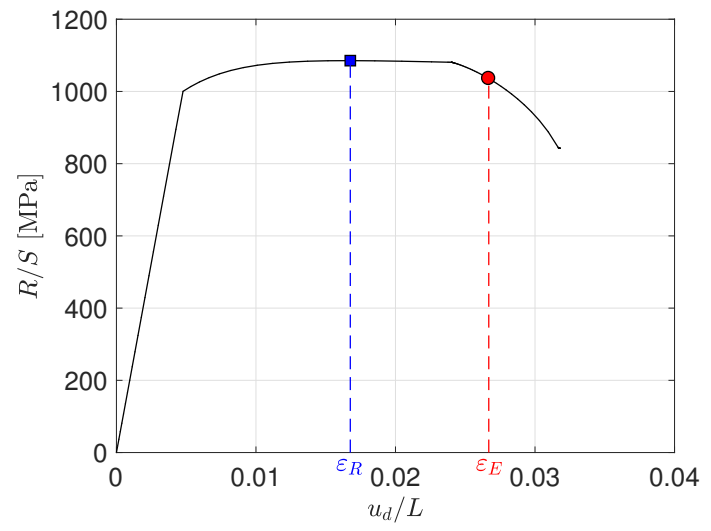

(a) von Mises

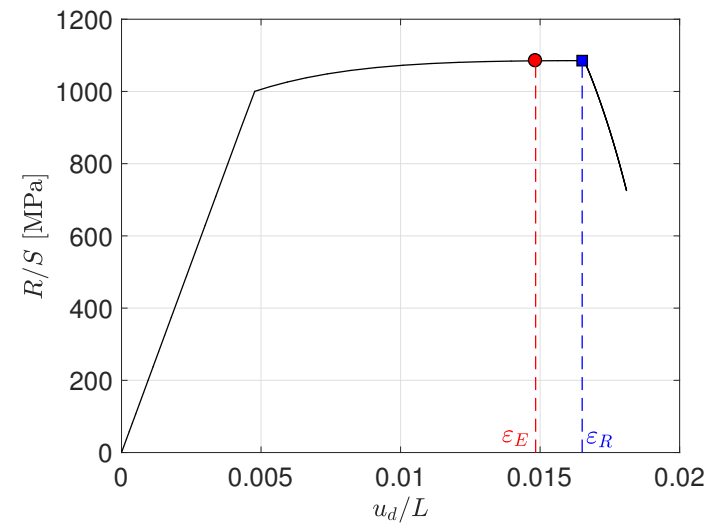

(b) Tresca

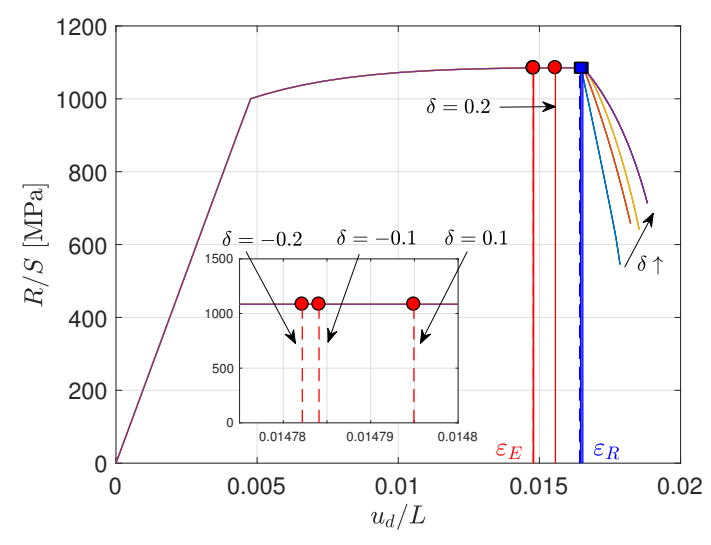

(c) $\delta$-Tresca

Figure 17: Tension of a thin plate: graphs of the force resultant $R / S$ with respect to the prescribed deformation $u_{d} / L$. The prescribed deformations $\varepsilon_{E}$ and $\varepsilon_{R}$ correspond to: the first occurrence of loss of ellipticity and to when $R$ reaches its maximum value. 

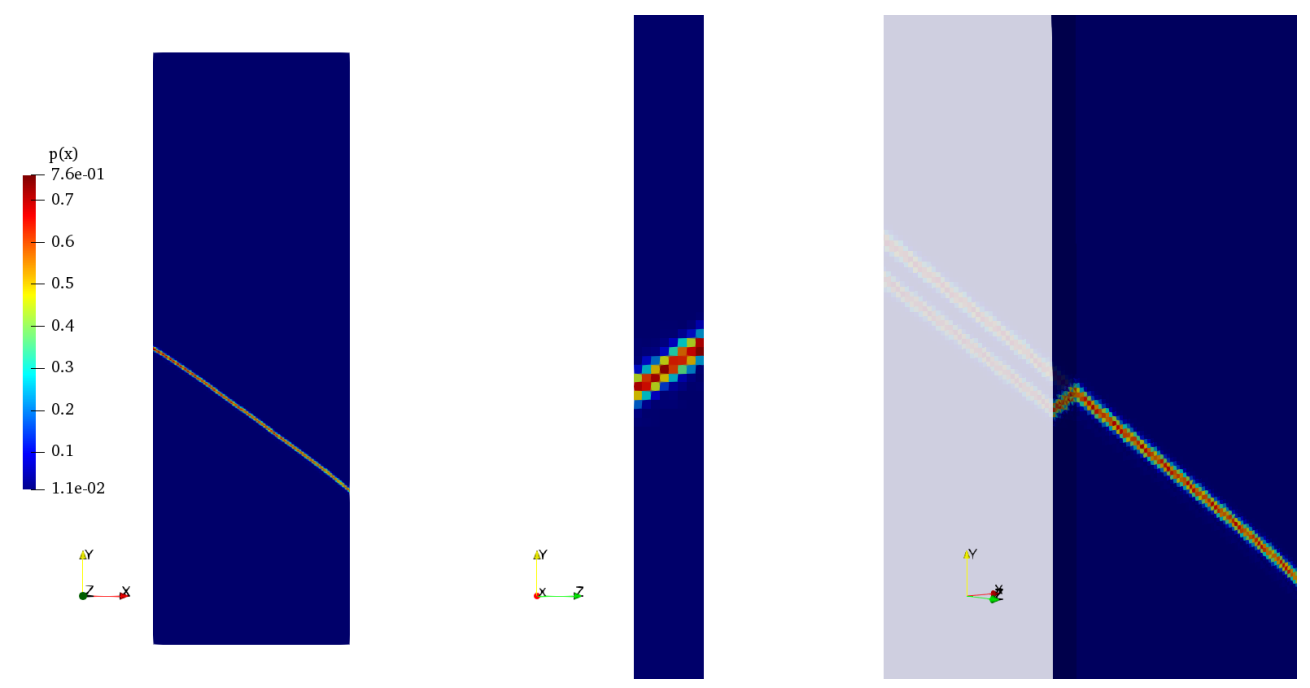

Figure 18: Cumulative plastic strain field in a Tresca plate for $u_{d} / L \approx 0.0175$. Three views of the plate are shown (from left to right): front view, side view, and clipped view inside the plate.

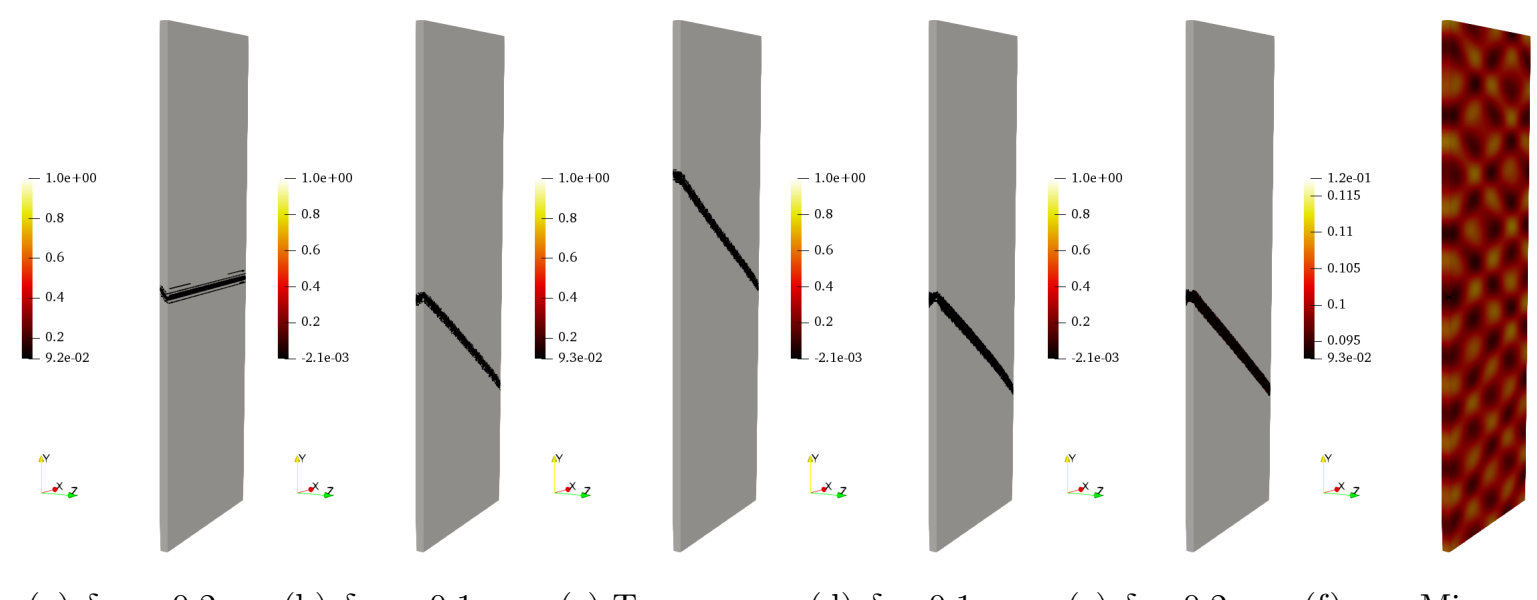
(a) $\delta=-0.2$
(b) $\delta=-0.1$
(c) Tresca
(d) $\delta=0.1$
(e) $\delta=0.2$
(f) von Mises

Figure 19: Tension of a thin plate: loss of ellipticity indicators for various plastic models and a prescribed displacement $u_{d} / L \approx 0.0175$. 


\subsection{Experimental tubular specimen undergoing tension and torsion}

In this last section, we consider the experimental tubular specimen shown in Fig.12(c). The objective of this example is to show that the proposed analysis is applicable to realistic experimental samples under complex loading conditions. Three different boundary value problems are considered. The first one consists in a pure torsion test where a relative rotation $\theta$ is applied to the top and bottom surfaces. The second configuration is a simple tension test where a vertical displacement is applied to the top surface. And finally, the third scenario is obtained by combining these tension and torsion tests. As in the previous numerical illustrations, a slightly softening behavior is enforced by taking $S=-1000 \mathrm{MPa}$ (see Eq. (105)). However, in contrast to the previous cases in sections 5.2 and 5.3, no imperfection is inserted in the finite element mesh. The following subsections comment the finite element simulations displayed in Fig.21 (pure torsion), Fig.22 (simple tension), Fig .23 (combined torsion-tension). All the snapshots were taken at the time-step at which ellipticity is lost for the first time during the simulations. The reader is referred to Fig.20 where graphs of the resultant moments and forces are shown, together with the first occurrences of loss of ellipticity. The results can be compared to the corresponding experimental data provided by [48] for ultra-high strength steel which does not exhibit significant damage prior to shear band localization and final fracture. It is proposed that Tresca-like constitutive models would be more suitable than the standard von Mises criterion to capture these localization events.

\subsubsection{Experimental tubular specimen undergoing pure torsion}

Graphs of the resultant moment with respect to the rotation angle are shown in the left panel of Fig.20 in the case of pure torsion, and snapshots of the cumulative plastic strains and loss of ellipticity indicators are provided in Fig.21. In this scenario, the stress tensor lies on the smooth portion of the $\delta$-Tresca yield surfaces. It can be observed that the Tresca and von Mises models lead to loss of ellipticity almost simultaneously when the resultant moment reaches its maximum value. This is due to the fact that their normals to the yield surfaces and critical hardening moduli coincide in pure shear. In contrast, the $\delta$-Tresca 
models with $\delta=-0.2$ or $\delta=0.2$ allow for postponing loss of ellipticity. These observations are in agreement with the analysis carried out in section 4.2 .1 .

\subsubsection{Experimental tubular specimen undergoing simple tension}

Graphs of the resultant force with respect to the prescribed displacement are shown in the right panel of Fig.20. Only a positive value for $\delta$ is considered since negative values lead to a material that never reaches ellipticity when it undergoes simple tension (see Fig. 8). As in the previous examples, it can be seen that the Tresca and $\delta$-Tresca models lose ellipticity earlier than the von Mises model. The $\delta$-Tresca and von Mises model exhibits similar cumulative plastic strain fields and loss of ellipticity indicators as it can be seen in Fig.22, The Tresca test tube plastifies in a similar fashion but with different magnitudes. Note that these snapshots are taken when the structure loses ellipticity for the first time during simulation and for further time steps, the three models exhibit different localization bands. The von Mises model was expected to remain elliptic but due to the geometry, the material does not undergo simple tension, even in the region of interest.

\subsubsection{Experimental tubular specimen undergoing combined torsion-tension}

In this last scenario, a $1 \mathrm{~mm}$ vertical prescribed displacement and a $10^{\circ}$ rotation along the test tube axis are applied to the top surface. Snapshots of the cumulative plastic strain fields and the loss of ellipticity indicators are shown in Fig.23. The von Mises model loses ellipticity for $u_{d}=0.0396 \mathrm{~mm}$ and $\theta=0.396^{\circ}$, while the Tresca and $\delta$-Tresca model lose ellipticity at times for $u_{d}=0.0275 \mathrm{~mm}, \theta=0.275^{\circ}$, and $u_{d}=0.0275 \mathrm{~mm}, \theta=0.273^{\circ}$, respectively. Each model exhibit similar localization patterns but with different magnitudes. While the von Mises model tends to localize on the inner surface of the specimen, the Tresca model localizes on the outer surface. The $\delta$-Tresca seems to stabilize the simulation as it leads to lower cumulative plastic strains when ellipticity is lost.

\section{Conclusion}

This work is concerned with the analysis of ellipticity when dealing with non-smooth yield surfaces. Towards this end, we consider a class of yield functions that are given by a linear 

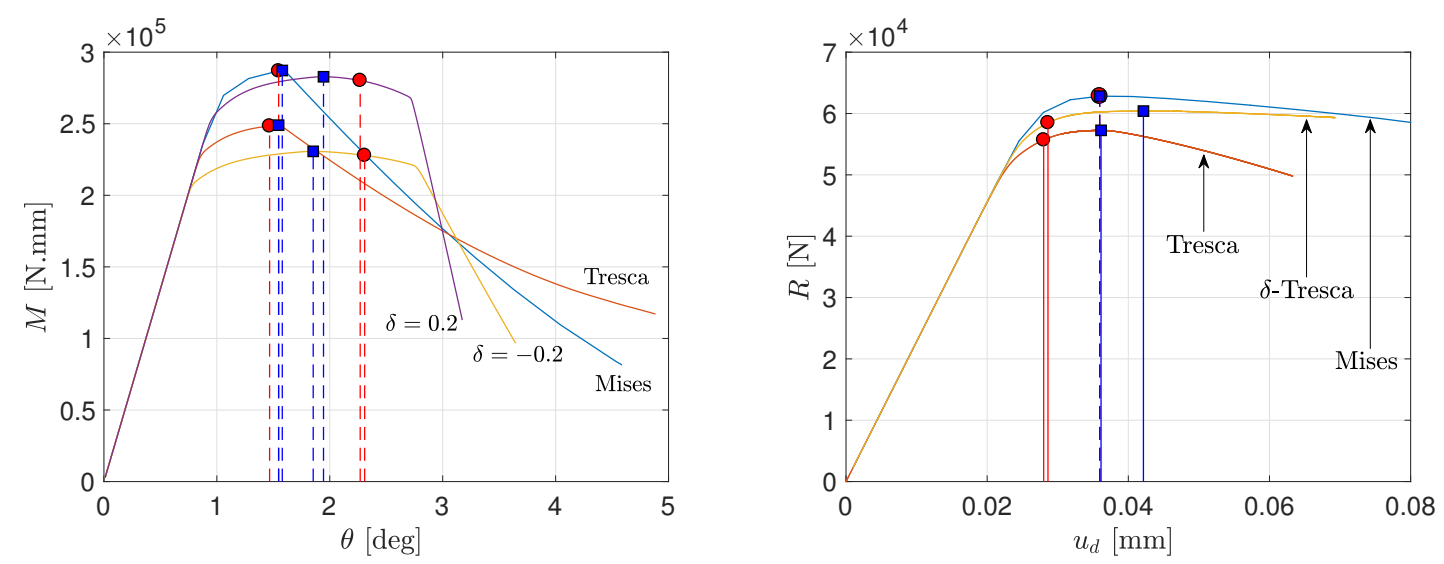

(a) Pure torsion: moments w.r.t the rotation angle.(b) Simple tension: reaction w.r.t the prescribed displacement.

Figure 20: Simple tension and pure torsion of a tubular specimen. Red bullets indicate loss of ellipticity and blue squares point out the maximum load.

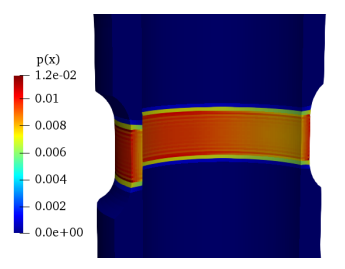

(a) von Mises

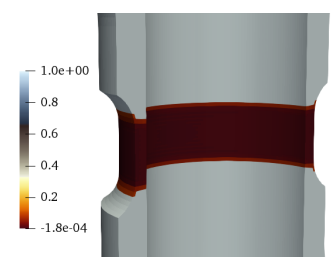

(e) von Mises

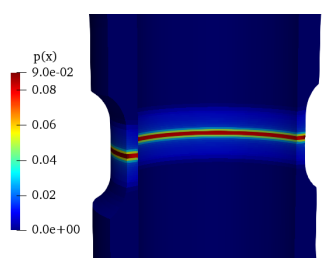

(b) $\delta=-0.2$

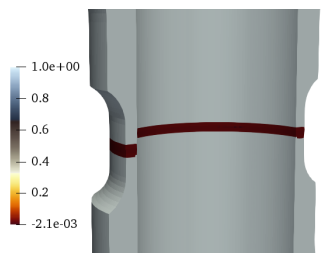

(f) $\delta=-0.2$

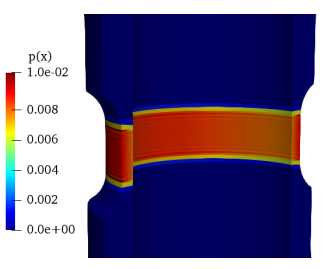

(c) $\delta=0$ (Tresca)

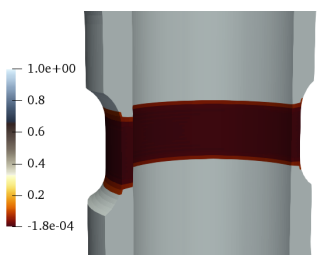

(g) $\delta=0$ (Tresca)

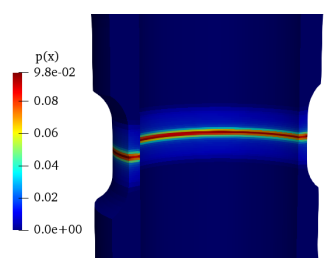

(d) $\delta=0.2$

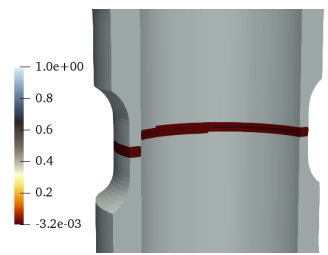

(h) $\delta=0.2$

Figure 21: Pure torsion test: cumulative plastic strain (top row) and loss of ellipticity indicator $\inf _{\mathbf{n}} \operatorname{det}\left(\mathbf{Q}_{e p}(\mathbf{n})\right)$ (bottom row) when ellipticity is lost for the first time during the simulations (Fig. 20(a)). 


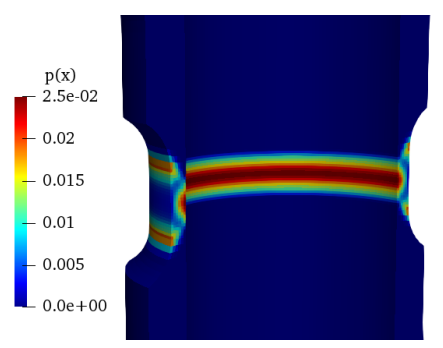

(a) von Mises

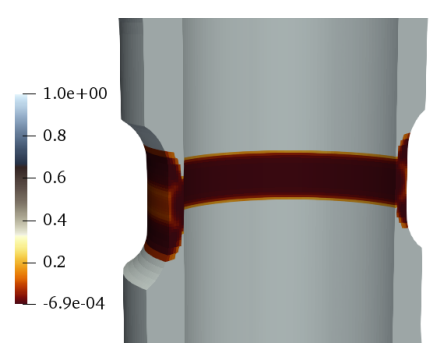

(d) von Mises

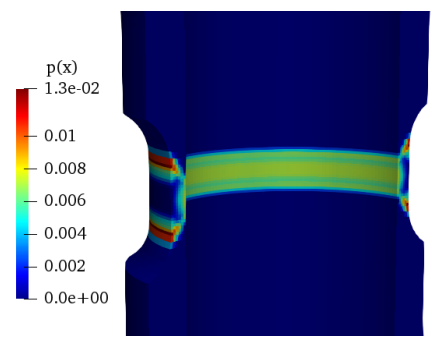

(b) Tresca

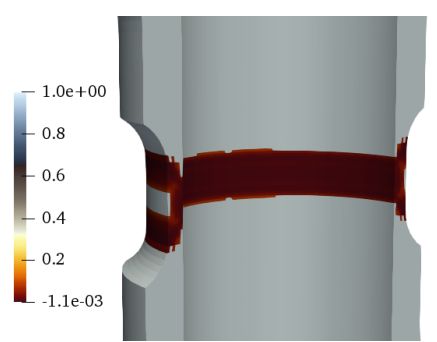

(e) Tresca

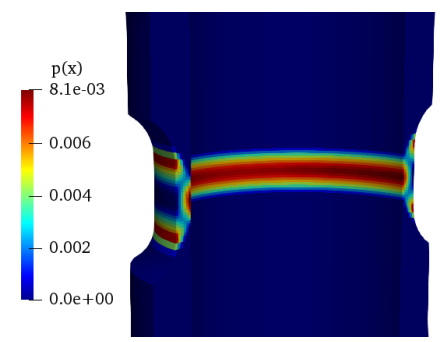

(c) $\delta=0.2$

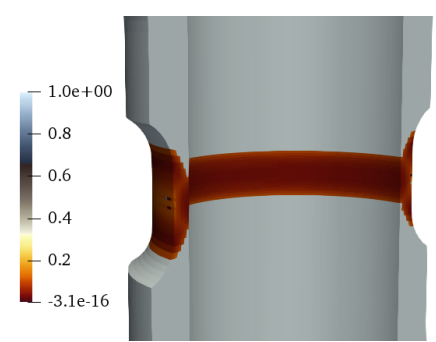

(f) $\delta=0.2$

Figure 22: Simple tension: cumulative plastic strains (top row) and loss of ellipticity indicator $\inf _{\mathbf{n}} \operatorname{det}\left(\mathbf{Q}_{e p}(\mathbf{n})\right)$ (bottom row) when ellipticity is lost for the first time during the simulations (Fig. 20 (b)). 


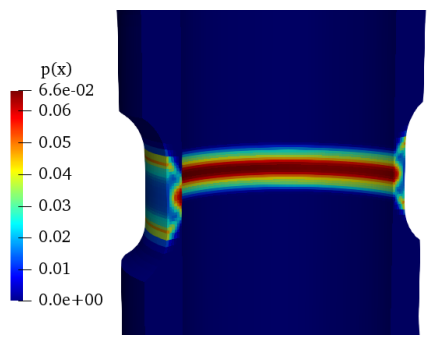

(a) von Mises

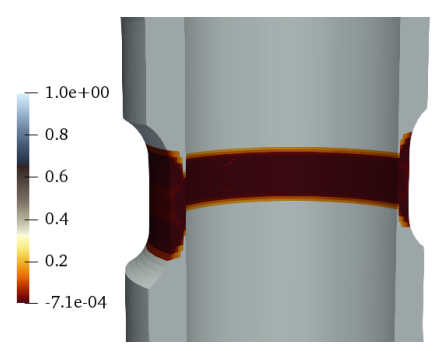

(d) von Mises

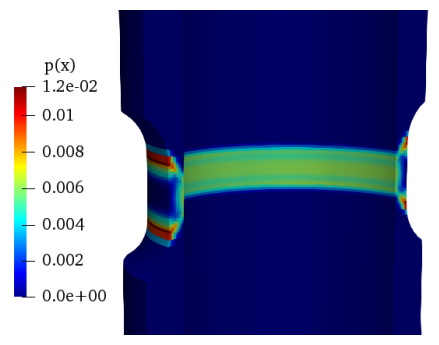

(b) Tresca

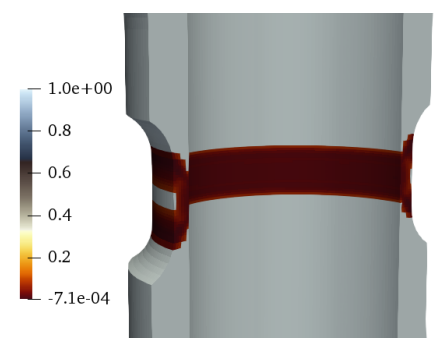

(e) Tresca

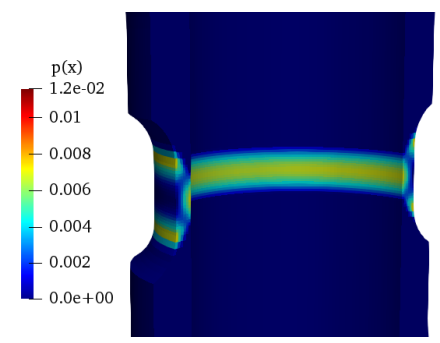

(c) $\delta=0.2$

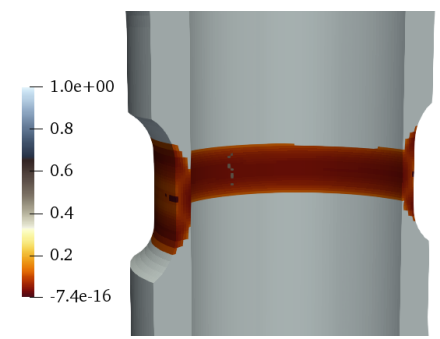

(f) $\delta=0.2$

Figure 23: Combined tension-torsion: cumulative plastic strains (top row) and loss of ellipticity indicator $\inf _{\mathbf{n}} \operatorname{det}\left(\mathbf{Q}_{e p}(\mathbf{n})\right)$ (bottom row) when ellipticity is lost for the first time during the simulations.

combination of the principal stresses. In principal stress space, yield functions belonging to such a family are non-smooth as they include two sharp corners on their surfaces, referred to as the left and right corners. For simplicity, three particular cases are considered, namely, the Tresca, $\delta$-Tresca, and $\tau$-Tresca models. The parameters $\delta$ and $\tau$ allow for modifying the positions and the shape of the corners. The following findings are made:

(a) The corners on the yield surface have a significant effect on the conditions for loss of ellipticity, i.e., they strongly affect the critical hardening modulus. In order to better understand this phenomenon, two new parametric families of yield functions are introduced. The parameter $\delta$ (resp. $\tau$ ) in the $\delta$-Tresca (resp. $\tau$-Tresca) model allows for increasing or decreasing the angle formed by the right corner (resp. left corner) of the yield surface.

(b) It is found that the presence of such corners allow for postponing of advancing loss of ellipticity. For instance, on the right corner of the $\delta$-Tresca yield surface, the critical 
hardening modulus can be negative or positive depending on the chosen value for $\delta$. In the particular case of the Tresca model, recovered for $\delta=0$, it is found that the critical hardening modulus vanishes.

(c) A numerical procedure for the detection of loss of ellipticity in multisurface plasticity is obtained by combining the recent algorithm proposed by [9] and the analysis proposed by 44. This numerical approach is used to determine the critical hardening moduli for all the considered non-smooth yield functions and to detect loss of ellipticity in finite element simulations.

(d) An explicit expression of the subdifferential of the non-smooth yield function is derived and generalize recent expressions obtained in the cases of the Tresca [39] and MohrCoulomb [40] models. This expression allows for expressing the flow rule in an abstract compact form and can be used for deriving an implicit integration algorithm.

(e) Finite element simulations on simple geometries undergoing simple tension show that the Tresca and $\delta$-Tresca models lead to loss of ellipticity much earlier than the commonly used von Mises model. More specifically, the von Mises model loses ellipticity for unrealistic deformations that are out of the range of the small perturbations assumption. In contrast, the Tresca and $\delta$-Tresca models may lose ellipticity for much lower deformations.

(f) Finite element simulations on a realistic structure show that the choice of the yield function can lead to drastic differences in terms of localization and loss of ellipticity. In all cases, it is seen that the non-smooth yield functions lose ellipticity earlier than the von Mises model and they also lead to different localization patterns and localization magnitudes.

These findings suggest that the choice of the yield function, and its smoothness, is of primary importance for structural computations. Strain localization observed in many engineering alloys (see, e.g., [48, 9]) can lead to slant fracture that cannot be properly described 
by existing ductile fracture models. The von Mises model and more generally smooth yield functions that are used in the industry, do not properly account for such localization modes under tension. We anticipate that more realistic models should incorporate corners, emerging from polycrystal plasticity. As demonstrated in the present work, such models can predict earlier strain localization events.

In future works, it is planned to study the influence of kinematic hardening on material instabilities, incorporate large deformation in the present anlaysis, and investigate the influence of corners in polycrystal plasticity.

\section{Acknowledgement}

The financial support of the DGAC (Direction générale de l'Aviation civile) is gratefully acknowledged.

\section{Appendix A. Subdifferential set for the family of yield criteria linear in principal stresses}

In this section, an explicit expression of the subdifferential set $\partial_{\boldsymbol{\sigma}} \phi \sqrt{75}$ is provided for the yield function defined by Eq. (68). To the authors' best knowledge, such expressions are only available for the Tresca [39] and Mohr-Coulomb [40] models. Based on the previous work of [51], two approaches are presented by [39], namely, a direct approach that starts from the definition (75), and an indirect approach that uses corollary results of convex analysis (see, e.g., [52, 53]). In the work of [40], the expression of the subdifferential set is obtained starting from its definition (75) in the case of a Mohr-Coulomb criterion. Here, we adopt an indirect approach. The yield function $(68)$ can be rewritten as

$$
\phi(\boldsymbol{\sigma}, R)=\left(a_{1}-a_{2}\right) \sigma_{1}(\boldsymbol{\sigma})+a_{2} \operatorname{Tr}(\boldsymbol{\sigma})+\left(a_{2}-a_{3}\right)\left(-\sigma_{3}(\boldsymbol{\sigma})\right),
$$

where it is recalled that $a_{1}>0, a_{3}<0, a_{1}>a_{2}>a_{3}$, and $\sigma_{1}(\boldsymbol{\sigma}) \geq \sigma_{2}(\boldsymbol{\sigma}) \geq \sigma_{3}(\boldsymbol{\sigma})$. According to [51, 53, the functions $\boldsymbol{\sigma} \mapsto \sigma_{1}(\boldsymbol{\sigma})$ and $\boldsymbol{\sigma} \mapsto\left(-\sigma_{3}(\boldsymbol{\sigma})\right)$ are both convex. The yield function (A.1) being given by a sum of three convex functions, it can be deduced 
[52, 53] that its subdifferential takes the form

$$
\partial_{\boldsymbol{\sigma}} \phi(\boldsymbol{\sigma}, R)=\left(a_{1}-a_{2}\right) \partial_{\boldsymbol{\sigma}} \sigma_{1}(\boldsymbol{\sigma})+a_{2} \partial_{\boldsymbol{\sigma}} \operatorname{Tr}(\boldsymbol{\sigma})+\left(a_{2}-a_{3}\right) \partial_{\boldsymbol{\sigma}}\left(-\sigma_{3}(\boldsymbol{\sigma})\right) .
$$

The subdifferential set of the largest eigenvalue is given by [51, 53.

$$
\partial_{\boldsymbol{\sigma}} \sigma_{1}(\boldsymbol{\sigma})=\left\{\boldsymbol{\xi}=\sum_{i=1}^{3} \xi_{i} \mathbf{m}_{i} \otimes \mathbf{m}_{i} \mid \boldsymbol{\xi}: \boldsymbol{\sigma}=\sigma_{1}(\boldsymbol{\sigma}), \operatorname{Tr}(\boldsymbol{\xi})=1\right\}
$$

and can be reduced to

$$
\partial_{\boldsymbol{\sigma}} \sigma_{1}(\boldsymbol{\sigma})=\left\{\boldsymbol{\xi}=\sum_{i=1}^{3} \xi_{i} \mathbf{m}_{i} \otimes \mathbf{m}_{i} \mid\left(\sigma_{1}-\sigma_{2}\right)\left(\xi_{1}-1\right)+\left(\sigma_{2}-\sigma_{3}\right)\left(-\xi_{3}\right)=0, \operatorname{Tr}(\boldsymbol{\xi})=1\right\} .
$$

The subdifferential of the smallest eigenvalue is such that $\partial_{\boldsymbol{\sigma}}\left(-\sigma_{3}(\boldsymbol{\sigma})\right)=-\partial_{\boldsymbol{\sigma}} \sigma_{1}(-\boldsymbol{\sigma})$. Using Eq. A.3) and the relationship $\sigma_{1}(-\boldsymbol{\sigma})=-\sigma_{3}(\boldsymbol{\sigma})$, it can be deduced that

$$
\partial_{\boldsymbol{\sigma}}\left(-\sigma_{3}(\boldsymbol{\sigma})\right)=\left\{\boldsymbol{\eta}=\sum_{i=1}^{3} \eta_{i} \mathbf{m}_{i} \otimes \mathbf{m}_{i} \mid\left(\sigma_{1}-\sigma_{2}\right) \eta_{1}+\left(\sigma_{2}-\sigma_{3}\right)\left(-1-\eta_{3}\right)=0, \operatorname{Tr}(\boldsymbol{\eta})=-1\right\} .
$$

Finally, the function $\boldsymbol{\sigma} \mapsto \operatorname{Tr}(\boldsymbol{\sigma})$ is smooth and its subdifferential is given by the singleton $\{\mathbf{I}\}$ containing the identity tensor. It follows that any tensor $\mathbf{W} \in \partial_{\boldsymbol{\sigma}} \phi(\boldsymbol{\sigma}, R)$ is given by

$$
\mathbf{W}=\left(a_{1}-a_{2}\right) \boldsymbol{\xi}+a_{2} \sum_{i=1}^{3} \mathbf{m}_{i} \otimes \mathbf{m}_{i}+\left(a_{2}-a_{3}\right) \boldsymbol{\eta}, \quad \boldsymbol{\xi} \in \partial_{\boldsymbol{\sigma}} \sigma_{1}(\boldsymbol{\sigma}), \quad \boldsymbol{\eta} \in \partial_{\boldsymbol{\sigma}}\left(-\sigma_{3}(\boldsymbol{\sigma})\right) .
$$

A compact expression of the subdifferential set can be obtained by noticing that the coefficients $W_{1}, W_{2}, W_{3}$ such that $\mathbf{W}=\sum_{i=1}^{3} W_{i} \mathbf{m}_{i} \otimes \mathbf{m}_{i}$ satisfy

$$
\left(\sigma_{1}-\sigma_{2}\right)\left(W_{1}-a_{1}\right)+\left(\sigma_{2}-\sigma_{3}\right)\left(a_{3}-W_{3}\right)=0, \quad W_{1}+W_{2}+W_{3}=a_{1}+a_{2}+a_{3}
$$

Hence, Eq. A.2 is also given by

$\partial_{\boldsymbol{\sigma}} \phi(\boldsymbol{\sigma}, R)=\left\{\mathbf{W}=\sum_{i=1}^{3} W_{i} \mathbf{m}_{i} \otimes \mathbf{m}_{i} \mid\left(\sigma_{1}-\sigma_{2}\right)\left(W_{1}-a_{1}\right)+\left(\sigma_{2}-\sigma_{3}\right)\left(a_{3}-W_{3}\right)=0, \operatorname{Tr}(\mathbf{W})=a_{1}+a_{2}+a_{3}\right\}$.

Using either Eq. A.2 or Eq. A.8) and by considering stress tensors on the smooth portion, left corner, or right corner of the yield surface, it can be verified that the abstract flow rule $\dot{\boldsymbol{\varepsilon}}^{p} \in \dot{\gamma} \partial_{\boldsymbol{\sigma}} \phi(\boldsymbol{\sigma}, R)$ is equivalent to the multisurface flow rule presented in section 4.1 . 


\section{Appendix B. Useful expressions for the analysis of loss of ellipticity}

The analysis conducted in section 4.2 .3 requires the expressions of the matrices $\left[\pi_{r}^{(\delta)}(\mathbf{n})\right]$, $\left[\pi_{l}^{(\delta)}(\mathbf{n})\right],\left[\pi_{r}^{(\tau)}(\mathbf{n})\right]$, and $\left[\pi_{l}^{(\tau)}(\mathbf{n})\right]$. The entries of these matrices can be obtained using Eqs. (42) together with (79). According to Eq. (79), the second-order tensors $\mathbf{N}^{1}, \mathbf{N}^{2}$, and $\mathbf{N}^{3}$ are given by

$$
\mathbf{N}^{1}=\left[\begin{array}{l}
a_{1} \\
a_{2} \\
a_{3}
\end{array}\right]_{\left(\mathbf{m}_{i}\right)_{i=1}^{3}}, \quad \mathbf{N}^{2}=\left[\begin{array}{l}
a_{2} \\
a_{1} \\
a_{3}
\end{array}\right]_{\left(\mathbf{m}_{i}\right)_{i=1}^{3}}, \quad \mathbf{N}^{3}=\left[\begin{array}{l}
a_{1} \\
a_{3} \\
a_{2}
\end{array}\right]_{\left(\mathbf{m}_{i}\right)_{i=1}^{3}},
$$

where $\left\{\mathbf{m}_{i}\right\}_{i=1}^{3}$ denotes the set of principal stress directions. For arbitrary values of the parameters $a_{1}, a_{2}, a_{3}$ in $\mathcal{C}$ (see Eq. (69)), the entries of the matrix $[\pi(\mathbf{n})]$ by plugging Eq. (B.1) into Eq. (42), i.e., into

$$
\hat{\pi}_{I J}\left(n_{1}, n_{2}, n_{3}\right)=-\frac{4 \mu(\lambda+\mu)}{\lambda+2 \mu} \sum_{i=1}^{3} n_{i}^{2} N_{i}^{K} \sum_{j=1}^{3} n_{j}^{2} N_{j}^{J}+4 \mu \sum_{i=1}^{3} N_{i}^{K} N_{i}^{L} n_{i}^{2}, \quad \mathbf{n}=\sum_{k=1}^{3} n_{k} \mathbf{m}_{k},
$$

where $(I, J) \in\{1,2\}^{2}$. On the left corner of the yield surface, $(K, L) \in \mathbb{J} \times \mathbb{J}$ with $\mathbb{J}=\{1,2\}$. On the right corner, the set of active mechanisms is given by $\mathbb{J}=\{1,3\}$. In the case of the $\delta$-Tresca model, one has $a_{1}=1, a_{2}=-\delta$, and $a_{3}=-1+\delta$. Hence, the second-order tensors in Eq. B.1) reduce to

$$
\mathbf{N}^{1}=\left[\begin{array}{c}
1 \\
-\delta \\
-1+\delta
\end{array}\right]_{\left(\mathbf{m}_{i}\right)_{i=1}^{3}}, \quad \mathbf{N}^{2}=\left[\begin{array}{c}
-\delta \\
1 \\
-1+\delta
\end{array}\right]_{\left(\mathbf{m}_{i}\right)_{i=1}^{3}}, \quad \mathbf{N}^{3}=\left[\begin{array}{c}
1 \\
-1+\delta \\
-\delta
\end{array}\right]_{\left(\mathbf{m}_{i}\right)_{i=1}^{3}}
$$

Using the second-order tensors $\mathbf{N}^{1}$ and $\mathbf{N}^{2}$, it is found that the entries of the matrix $\left[\pi_{l}^{\delta}(\mathbf{n})\right]$ are given by

$$
\begin{aligned}
& {\left[\hat{\pi}_{l}^{\delta}\left(n_{1}, n_{2}, n_{3}\right)\right]_{11}=-\frac{4 \mu(\lambda+\mu)}{\lambda+2 \mu}\left(n_{1}^{2}-\delta n_{2}^{2}-(1-\delta) n_{3}^{2}\right)^{2}+4 \mu\left(n_{1}^{2}+\delta^{2} n_{2}^{2}+(1-\delta)^{2} n_{3}^{2}\right),} \\
& {\left[\hat{\pi}_{l}^{\delta}\left(n_{1}, n_{2}, n_{3}\right)\right]_{22}=-\frac{4 \mu(\lambda+\mu)}{\lambda+2 \mu}\left(-\delta n_{1}^{2}+n_{2}^{2}-(1-\delta) n_{3}^{2}\right)^{2}+4 \mu\left(\delta^{2} n_{1}^{2}+n_{2}^{3}+(1-\delta)^{2} n_{3}^{2}\right),}
\end{aligned}
$$


and $\left[\hat{\pi}_{l}^{\delta}\right]_{21}=\left[\hat{\pi}_{l}^{\delta}\right]_{12}$ with

$$
\begin{aligned}
{\left[\hat{\pi}_{l}^{\delta}\left(n_{1}, n_{2}, n_{3}\right)\right]_{12}=} & -\frac{4 \mu(\lambda+\mu)}{\lambda+2 \mu}\left(n_{1}^{2}-\delta n_{2}^{2}-(1-\delta) n_{3}^{2}\right)\left(-\delta n_{1}^{2}+n_{2}^{2}-(1-\delta) n_{3}^{2}\right) \\
& +4 \mu\left(n_{1}^{2}+\delta^{2} n_{2}^{2}+(1-\delta)^{2} n_{3}^{2}\right)\left(\delta^{2} n_{1}^{2}+n_{2}^{3}+(1-\delta)^{2} n_{3}^{2}\right) .
\end{aligned}
$$

Similarly, using the second-order tensors $\mathbf{N}^{1}$ and $\mathbf{N}^{3}$, the following entries of the matrix $\left[\pi_{r}^{\delta}(\mathbf{n})\right]$ are found:

$$
\begin{aligned}
& {\left[\hat{\pi}_{r}^{\delta}\left(n_{1}, n_{2}, n_{3}\right)\right]_{11}=-\frac{4 \mu(\lambda+\mu)}{\lambda+2 \mu}\left(n_{1}^{2}-\delta n_{2}^{2}-(1-\delta) n_{3}^{2}\right)^{2}+4 \mu\left(n_{1}^{2}+\delta^{2} n_{2}^{2}+(1-\delta)^{2} n_{3}^{2}\right),} \\
& {\left[\hat{\pi}_{r}^{\delta}\left(n_{1}, n_{2}, n_{3}\right)\right]_{22}=-\frac{4 \mu(\lambda+\mu)}{\lambda+2 \mu}\left(n_{1}^{2}-(1-\delta) n_{2}^{2}-\delta n_{3}^{2}\right)^{2}+4 \mu\left(n_{1}^{2}+(1-\delta)^{2} n_{2}^{3}+\delta n_{3}^{2}\right),}
\end{aligned}
$$

and $\left[\hat{\pi}_{r}^{\delta}\right]_{21}=\left[\hat{\pi}_{r}^{\delta}\right]_{12}$ with

$$
\begin{aligned}
{\left[\hat{\pi}_{r}^{\delta}\left(n_{1}, n_{2}, n_{3}\right)\right]_{12}=} & -\frac{4 \mu(\lambda+\mu)}{\lambda+2 \mu}\left(n_{1}^{2}-\delta n_{2}^{2}-(1-\delta) n_{3}^{2}\right)\left(n_{1}^{2}-(1-\delta) n_{2}^{2}-\delta n_{3}^{2}\right) \\
& +4 \mu\left(n_{1}^{2}+\delta^{2} n_{2}^{2}+(1-\delta)^{2} n_{3}^{2}\right)\left(n_{1}^{2}+(1-\delta)^{2} n_{2}^{3}+\delta n_{3}^{2}\right) .
\end{aligned}
$$

In the case of the $\tau$-Tresca model, the parameters $a_{1}, a_{2}, a_{3}$ are given by $a_{1}=1-\tau, a_{2}=\tau$, and $a_{3}=-1$. As a result, the second-order tensors $\mathbf{N}^{1}, \mathbf{N}^{2}$, and $\mathbf{N}^{3}$ reduce to

$$
\mathbf{N}^{1}=\left[\begin{array}{c}
1-\tau \\
\tau \\
-1
\end{array}\right]_{\left(\mathbf{m}_{i}\right)_{i=1}^{3}}, \quad \mathbf{N}^{2}=\left[\begin{array}{c}
\tau \\
1-\tau \\
-1
\end{array}\right]_{\left(\mathbf{m}_{i}\right)_{i=1}^{3}}, \quad \mathbf{N}^{3}=\left[\begin{array}{c}
1-\tau \\
-1 \\
\tau
\end{array}\right]_{\left(\mathbf{m}_{i}\right)_{i=1}^{3}}
$$

It can be deduced that the entries of the matrix $\left[\pi_{l}^{\tau}(\mathbf{n})\right]$ are given by

$$
\begin{aligned}
& {\left[\hat{\pi}_{l}^{\tau}\left(n_{1}, n_{2}, n_{3}\right)\right]_{11}=-\frac{4 \mu(\lambda+\mu)}{\lambda+2 \mu}\left((1-\tau) n_{1}^{2}+\tau n_{2}^{2}-n_{3}^{2}\right)^{2}+4 \mu\left((1-\tau)^{2} n_{1}^{2}+\tau^{2} n_{2}^{2}+n_{3}^{2}\right),} \\
& {\left[\hat{\pi}_{l}^{\tau}\left(n_{1}, n_{2}, n_{3}\right)\right]_{22}=-\frac{4 \mu(\lambda+\mu)}{\lambda+2 \mu}\left(\tau n_{1}^{2}+(1-\tau) n_{2}^{2}-n_{3}^{2}\right)^{2}+4 \mu\left(\tau^{2} n_{1}^{2}+(1-\tau)^{2} n_{2}^{3}+n_{3}^{2}\right),}
\end{aligned}
$$

and $\left[\hat{\pi}_{l}^{\tau}\right]_{21}=\left[\hat{\pi}_{l}^{\tau}\right]_{12}$ with

$$
\begin{aligned}
{\left[\hat{\pi}_{l}^{\tau}\left(n_{1}, n_{2}, n_{3}\right)\right]_{12}=} & -\frac{4 \mu(\lambda+\mu)}{\lambda+2 \mu}\left((1-\tau) n_{1}^{2}+\tau n_{2}^{2}-n_{3}^{2}\right)\left(\tau n_{1}^{2}+(1-\tau) n_{2}^{2}-n_{3}^{2}\right) \\
& +4 \mu\left((1-\tau)^{2} n_{1}^{2}+\tau^{2} n_{2}^{2}+n_{3}^{2}\right)\left(\tau^{2} n_{1}^{2}+(1-\tau)^{2} n_{2}^{3}+n_{3}^{2}\right) .
\end{aligned}
$$


Finally, on the right corner of the $\tau$-Tresca yield surface, one has

$$
\begin{aligned}
& {\left[\hat{\pi}_{r}^{\tau}\left(n_{1}, n_{2}, n_{3}\right)\right]_{11}=-\frac{4 \mu(\lambda+\mu)}{\lambda+2 \mu}\left((1-\tau) n_{1}^{2}+\tau n_{2}^{2}-n_{3}^{2}\right)^{2}+4 \mu\left((1-\tau)^{2} n_{1}^{2}+\tau^{2} n_{2}^{2}+n_{3}^{2}\right),} \\
& {\left[\hat{\pi}_{r}^{\tau}\left(n_{1}, n_{2}, n_{3}\right)\right]_{22}=-\frac{4 \mu(\lambda+\mu)}{\lambda+2 \mu}\left((1-\tau) n_{1}^{2}-n_{2}^{2}+\tau n_{3}^{2}\right)^{2}+4 \mu\left((1-\tau)^{2} n_{1}^{2}+n_{2}^{3}+\tau^{2} n_{3}^{2}\right),}
\end{aligned}
$$

and $\left[\hat{\pi}_{r}^{\tau}\right]_{21}=\left[\hat{\pi}_{r}^{\tau}\right]_{12}$ with

$$
\begin{aligned}
{\left[\hat{\pi}_{r}^{\tau}\left(n_{1}, n_{2}, n_{3}\right)\right]_{12}=} & -\frac{4 \mu(\lambda+\mu)}{\lambda+2 \mu}\left((1-\tau) n_{1}^{2}+\tau n_{2}^{2}-n_{3}^{2}\right)\left((1-\tau) n_{1}^{2}-n_{2}^{2}+\tau n_{3}^{2}\right) \\
& +4 \mu\left((1-\tau)^{2} n_{1}^{2}+\tau^{2} n_{2}^{2}+n_{3}^{2}\right)\left((1-\tau)^{2} n_{1}^{2}+n_{2}^{3}+\tau^{2} n_{3}^{2}\right) .
\end{aligned}
$$

\section{References}

[1] R. Hill, Acceleration waves in solids, Journal of the Mechanics and Physics of Solids 10 (1) (1962) 1-16.

[2] J. Rice, The localization of plastic deformation, in: in: W.T. Koiter (Ed.), Theoretical and Applied Mechanics, North-Holland Publishing Company, 1976, pp. 207-220.

[3] E. Stein, P. Steinmann, C. Miehe, Instability phenomena in plasticity: modelling and computation, Computational mechanics 17 (1-2) (1995) 74-87.

[4] Q. Nguyen, Stability and nonlinear solid mechanics, Wiley, 2000.

[5] D. Bigoni, Nonlinear solid mechanics: bifurcation theory and material instability, Cambridge University Press, 2012

[6] H. Petryk, Material instabilities in elastic and plastic solids, Vol. 414, Springer, 2014.

[7] D. Bigoni, T. Hueckel, Uniqueness and localization-I. associative and non-associative elastoplasticity, International Journal of Solids and structures 28 (2) (1991) 197-213.

[8] J. Mosler, Numerical analyses of discontinuous material bifurcation: Strong and weak discontinuities, Computer Methods in Applied Mechanics and Engineering 194 (9-11) (2005) 979-1000.

[9] M. Al Kotob, C. Combescure, M. Mazière, T. Rose, S. Forest, A general and efficient multi-start algorithm for the detection of loss of ellipticity in elastoplastic structures, International Journal for Numerical Methods in Engineering 121 (2019) 842-866.

[10] G. Cailletaud, A micromechanical approach to inelastic behaviour of metals, International Journal of Plasticity 8 (1992) 55-73.

[11] P. Pilvin, The contribution of micromechanical approaches to the modelling of inelastic behaviour, in: A. Pineau, G. Cailletaud, T. Lindley (Eds.), 4th International Conference on Biaxial/multiaxial Fatigue, Saint-Germain, France, Vol. 1, ESIS, 1994, pp. 31-46.

[12] J. Christoffersen, J. Hutchinson, A class of phenomenological corner theories of plasticity, Journal of the Mechanics and Physics of Solids 27 (5-6) (1979) 465-487. 
[13] J. Hutchinson, V. Tvergaard, Surface instabilities on statically strained plastic solids, International Journal of Mechanical Sciences 22 (6) (1980) 339-354.

[14] J. Hutchinson, V. Tvergaard, Shear band formation in plane strain, International Journal of Solids and Structures 17 (5) (1981) 451-470.

[15] V. Tvergaard, A. Needleman, K. Lo, Flow localization in the plane strain tensile test, Journal of the Mechanics and Physics of Solids 29 (2) (1981) 115-142.

[16] N. Triantafyllidis, A. Needleman, V. Tvergaard, On the development of shear bands in pure bending, International Journal of Solids and Structures 18 (2) (1982) 121-138.

[17] H. Petryk, K. Thermann, On discretized plasticity problems with bifurcations, International journal of solids and structures 29 (6) (1992) 745-765.

[18] H. Petryk, K. Thermann, Post-critical plastic deformation in incrementally nonlinear materials, Journal of the Mechanics and Physics of Solids 50 (5) (2002) 925-954.

[19] T. Hughes, F. Shakib, Pseudo-corner theory: A simple enhancement of J2-flow theory for applications involving non-proportional loading, Engineering computations 3 (2) (1986) 116-120.

[20] J. Simo, A J2-flow theory exhibiting a corner-like effect and suitable for large-scale computation, Computer methods in applied mechanics and engineering 62 (2) (1987) 169-194.

[21] L. Rønning, O. Hopperstad, P. Larsen, Numerical study of the effects of constitutive models on plastic buckling of plate elements, European Journal of Mechanics-A/Solids 29 (4) (2010) 508-522.

[22] M. Kuroda, V. Tvergaard, A phenomenological plasticity model with non-normality effects representing observations in crystal plasticity, Journal of the Mechanics and Physics of Solids 49 (6) (2001) 12391263.

[23] M. Kuroda, A higher-order strain gradient plasticity theory with a corner-like effect, International Journal of Solids and Structures 58 (2015) 62-72.

[24] M. Kuroda, A strain-gradient plasticity theory with a corner-like effect: a thermodynamics-based extension, International Journal of Fracture 200 (1-2) (2016) 115-125.

[25] W. Koiter, Stress-strain relations, uniqueness and variational theorems for elastic-plastic materials with a singular yield surface, Quarterly of applied mathematics 11 (3) (1953) 350-354.

[26] J. Mandel, Généralisation de la théorie de plasticité de W.T. koiter, International Journal of Solids and structures 1 (3) (1965) 273-295.

[27] J. Simo, J. Kennedy, S. Govindjee, Non-smooth multisurface plasticity and viscoplasticity. loading/unloading conditions and numerical algorithms, International Journal for Numerical Methods in Engineering 26 (10) (1988) 2161-2185.

[28] D. Perić, E. de Souza Neto, A new computational model for Tresca plasticity at finite strains with an optimal parametrization in the principal space, Computer Methods in Applied Mechanics and Engi- 
neering 171 (3-4) (1999) 463-489.

[29] E. de Souza Neto, D. Peric, D. Owen, Computational methods for plasticity: theory and applications, John Wiley \& Sons, 2011.

[30] F. Karaoulanis, Implicit numerical integration of nonsmooth multisurface yield criteria in the principal stress space, Archives of Computational Methods in Engineering 20 (3) (2013) 263-308.

[31] J.-J. Moreau, Application of convex analysis to the treatment of elastoplastic systems, in: Applications of methods of functional analysis to problems in mechanics, Springer, 1976, pp. 56-89.

[32] P. Suquet, Sur les équations de la plasticité: existence et régularité des solutions, J. Mécanique 20 (1) (1981) 3-39.

[33] W. Han, B. Reddy, Plasticity: mathematical theory and numerical analysis, Vol. 9, Springer Science \& Business Media, 2012.

[34] C. Carstensen, K. Hackl, A. Mielke, Non-convex potentials and microstructures in finite-strain plasticity, Proceedings of the royal society of London. Series A: mathematical, physical and engineering sciences 458 (2018) (2001) 299-317.

[35] A. Mielke, Existence of minimizers in incremental elasto-plasticity with finite strains, SIAM journal on mathematical analysis 36 (2) (2004) 384-404.

[36] S. Sysala, M. Čermák, T. Koudelka, J. Kruis, J. Zeman, R. Blaheta, Subdifferential-based implicit return-mapping operators in computational plasticity, ZAMM-Journal of Applied Mathematics and Mechanics/Zeitschrift für Angewandte Mathematik und Mechanik 96 (11) (2016) 1318-1338.

[37] M. Yu, Twin shear stress yield criterion, International Journal of Mechanical Sciences 25 (1) (1983) $71-74$.

[38] S. Zhang, X. Jiang, C. Xiang, L. Deng, Y. Li, Proposal and application of a new yield criterion for metal plastic deformation, Archive of Applied Mechanics 90 (2020) 1705-1722.

[39] Q.-C. He, C. Vallée, C. Lerintiu, Explicit expressions for the plastic normality-flow rule associated to the Tresca yield criterion, Zeitschrift für angewandte Mathematik und Physik ZAMP 56 (2) (2005) $357-366$.

[40] S. Sysala, M. Čermák, T. Ligurskỳ, Subdifferential-based implicit return-mapping operators in MohrCoulomb plasticity, ZAMM-Journal of Applied Mathematics and Mechanics/Zeitschrift für Angewandte Mathematik und Mechanik 97 (12) (2017) 1502-1523.

[41] J. Simo, T. Hughes, Computational inelasticity, Vol. 7 of Interdisciplinary Applied Mathematics, Springer Science \& Business Media, 2006.

[42] B. Reddy, Mathematical Foundations of Elastoplastic Deformations in Solids, Encyclopedia of Computational Mechanics Second Edition, American Cancer Society, 2017.

[43] F. Clarke, Optimization and nonsmooth analysis, Vol. 5, SIAM, New-York, 1990. 
[44] E. Sawischlewski, P. Steinmann, E. Stein, Modelling and computation of instability phenomena in multisurface elasto-plasticity, Computational mechanics 18 (4) (1996) 245-258.

[45] P. Steinmann, On localization analysis in multisurface hyperelasto-plasticity, Journal of the Mechanics and Physics of Solids 44 (10) (1996) 1691-1713.

[46] A. Benallal, T. Berstad, T. Børvik, O. Hopperstad, Uniqueness, loss of ellipticity and localization for the time-discretized, rate-dependent boundary value problem with softening, International journal for numerical methods in engineering 84 (7) (2010) 864-882.

[47] R. Larsson, K. Runesson, Implicit integration and consistent linearization for yield criteria of the mohrcoulomb type, Mechanics of Cohesive-frictional Materials: An International Journal on Experiments, Modelling and Computation of Materials and Structures 1 (4) (1996) 367-383.

[48] C. Defaisse, M. Mazire, L. Marcin, J. Besson, Ductile fracture of an ultra-high strength steel under low to moderate stress triaxiality, Engineering Fracture Mechanics 194 (2018) $301-318$.

[49] R. Borja, K. Sama, P. Sanz, On the numerical integration of three-invariant elastoplastic constitutive models, Computer methods in applied mechanics and engineering 192 (9-10) (2003) 1227-1258.

[50] J. Besson, G. Cailletaud, J.-L. Chaboche, S. Forest, Non-linear mechanics of materials, Vol. 167, Springer Science \& Business Media, 2009.

[51] C. Vallée, Q.-C. He, C. Lerintiu, Convex analysis of the eigenvalues of a 3d second-order symmetric tensor, Journal of Elasticity 83 (2) (2006) 191-204.

[52] J. van Tiel, Convex analysis, John Wiley, 1984.

[53] A. Ruszczyński, Nonlinear Optimization, Princeton University Press, 2006. 